\title{
1 CXCR2 inhibition enables NASH-HCC immunotherapy
}

2

3 Jack Leslie ${ }^{1,2^{*}}$, John B. G. Mackey ${ }^{3^{*}}$, Thomas Jamieson ${ }^{3 *}$, Erik Ramon-Gil ${ }^{1,2}$, Thomas M. Drake ${ }^{3,4,5}$, 4 Frédéric Fercoq ${ }^{3}$, William Clark ${ }^{3}$, Kathryn Gilroy ${ }^{3}$, Ann Hedley ${ }^{3}$, Colin Nixon ${ }^{3}$, Saimir Luli ${ }^{1,2,6}$, Maja 5 Laszczewska ${ }^{1,2}$, Roser Pinyol ${ }^{7}$, Roger Esteban-Fabró 7,8 , Catherine E. Willoughby ${ }^{7}$, Philipp K. Haber ${ }^{8}$, 6 Carmen Andreu-Oller ${ }^{7,8}$, Mohammad Rahbari ${ }^{9}$, Chaofan Fan ${ }^{9}$, Dominik Pfister ${ }^{9}$, Shreya Raman ${ }^{10}$, Niall 7 Wilson ${ }^{1,2}$, Miryam Müller ${ }^{3}$, Amy Collins ${ }^{1,2}$, Daniel Geh ${ }^{1,2}$, Andrew Fuller ${ }^{11,12}$, David McDonald ${ }^{11,12}$, Gillian 8 Hulme $^{11,12}$, Andrew Filby ${ }^{11,12}$, Xabier Cortes-Lavaud ${ }^{3}$, Noha-Ehssan Mohamed ${ }^{3}$, Catriona A. Ford ${ }^{3}$, 9 Ximena L. Raffo Iraolagoitia ${ }^{3}$, Amanda J. McFarlane, Misiti McCain², Rachel Ridgway³, Edward W. Roberts $^{3}$, Simon T. Barry ${ }^{13}$, Gerard J. Graham ${ }^{14}$,Mathias Heikenwalder ${ }^{9,15}$, Helen L. Reeves ${ }^{2,16}$, Josep M. Llovet $^{7,8,17, \# \text {, Leo M. Carlin }}{ }^{3,4, \#}$, Thomas G. Bird ${ }^{3,5, \#, ~ O w e n ~ J . ~ S a n s o m ~}{ }^{3,4, \#}$, Derek A. Mann ${ }^{1,2,18,19 \# .}$

${ }^{1}$ Newcastle Fibrosis Research Group, Biosciences Institute, Faculty of Medical Sciences, Newcastle University, Newcastle upon Tyne, UK.

${ }^{2}$ The Newcastle University Centre for Cancer, Newcastle University, Newcastle upon Tyne, UK

${ }^{3}$ Cancer Research UK Beatson Institute, Glasgow, UK. ${ }^{4}$ Institute of Cancer Sciences, University of Glasgow, Glasgow, UK.

${ }^{5} \mathrm{MRC}$ Centre for Inflammation Research, The Queen's Medical Research Institute, University of Edinburgh, Edinburgh, UK.

${ }^{6}$ Preclinical In Vivo Imaging Facility, Faculty of Medical Sciences, Newcastle University, Newcastle upon Tyne, UK.

${ }^{7}$ Translational Research in Hepatic Oncology, Liver Unit, IDIBAPS, Hospital Clínic, University of Barcelona, Barcelona, Catalonia, Spain.

${ }^{8}$ Mount Sinai Liver Cancer Program, Division of Liver Diseases, Tisch Cancer Institute, Icahn School of Medicine at Mount Sinai New York, NY, USA.

${ }^{9}$ Division of Chronic Inflammation and Cancer, German Cancer Research Center (DKFZ), Heidelberg,

Germany.

${ }^{10}$ Department of Pathology, Newcastle upon Tyne NHS Foundation Trust, Newcastle upon Tyne, UK.

${ }^{11}$ Flow Cytometry Facility, Biosciences Institute, Faculty of Medical Sciences, Newcastle University, Newcastle upon Tyne, UK.

${ }^{12}$ Innovation, Methodology and Innovation (IMA) theme, Biosciences Institute, Faculty of Medical

32 Sciences, Newcastle University, Newcastle upon Tyne, UK.

${ }^{13}$ Bioscience, Early Oncology, AstraZeneca, Cambridge, UK. 
$34{ }^{14}$ Chemokine Research Group, Institute of Infection, Immunity and Inflammation, University of Glasgow, Glasgow, UK.

${ }^{15}$ Department of Surgery, University Hospital Mannheim, Medical Faculty Mannheim, University of Heidelberg, Germany.

${ }^{16}$ Translational and Clinical Research Institute, Faculty of Medical Sciences, Newcastle University,

39 Newcastle upon Tyne, UK.

$40 \quad{ }^{17}$ Institució Catalana de Recerca i Estudis Avançats (ICREA), Barcelona, Spain.

$41{ }^{18}$ Fibrofind Ltd, William Leech Building, Medical School, Newcastle University, Newcastle upon Tyne, 42 UK.

$43{ }^{19}$ Department of Gastroenterology and Hepatology, School of Medicine, Koç University, Istanbul, 44 Turkey.

$46{ }^{*}$ These authors contributed equally to this work.

47 \#email: Derek.Mann@ncl.ac.uk, o.sansom@beatson.gla.ac.uk, t.bird@beatson.gla.ac.uk, 48 leo.carlin@glasgow.ac.uk or jmllovet@clinic.cat

50 Conflict of interests: D.A.M. is a director of Fibrofind limited. J.L. and D.A.M. are shareholders in 51 Fibrofind limited. S.T.B. owns shares in AstraZeneca. O.J.S. receives funding from AstraZeneca and 52 Novartis. T.G.B. receives research funding support from AstraZeneca. J.M.L. receives research support 53 from Bayer HealthCare Pharmaceuticals, Eisai Inc, Bristol-Myers Squibb, Boehringer-Ingelheim and 54 Ipsen, and consulting fees from Eli Lilly, Bayer HealthCare Pharmaceuticals, Bristol-Myers Squibb, Eisai Inc, Celsion Corporation, Exelixis, Merck, Ipsen, Genentech, Roche, Glycotest, Nucleix, Sirtex, Mina Alpha Ltd and AstraZeneca. 


\section{Abstract}

58

Objective: Hepatocellular carcinoma (HCC) is increasingly associated with non-alcoholic steatohepatitis (NASH). HCC immunotherapy offers great promise; however, recent data suggests NASH-HCC may be less sensitive to conventional immune checkpoint inhibition (ICI). We hypothesised that targeting neutrophils using a CXCR2 small molecule inhibitor may sensitise NASH-HCC to ICI therapy.

Design: Neutrophil infiltration was characterised in human HCC and mouse models of HCC. Late-stage intervention with anti-PD1 and/or a CXCR2 inhibitor was performed in murine models of NASH-HCC. The tumour immune microenvironment was characterised by imaging mass cytometry, RNA-seq and flow cytometry.

Results: Neutrophils expressing CXCR2, a receptor crucial to neutrophil recruitment in acute-injury, are highly represented in human NASH-HCC. In models of NASH-HCC lacking response to $\mathrm{ICl}$, the combination of a CXCR2 antagonist with anti-PD1 suppressed tumour burden and extended survival. Combination therapy increased intratumoral $\mathrm{XCR} 1^{+}$dendritic cell activation and $\mathrm{CD} 8^{+} \mathrm{T}$ cell numbers which are associated with anti-tumoral immunity, this was confirmed by loss of therapeutic effect upon genetic impairment of myeloid cell recruitment, neutralisation of the XCR1-ligand XCL1 or depletion of $C D 8^{+} T$ cells. Therapeutic benefit was accompanied by an unexpected increase in tumourassociated neutrophils (TANs) which switched from a pro-tumor to anti-tumour progenitor-like neutrophil phenotype. Reprogrammed TANs were found in direct contact with $C D 8^{+} T$ cells in clusters that were enriched for the cytotoxic anti-tumoural protease granzyme B. Neutrophil reprogramming was not observed in the circulation indicative of the combination therapy selectively influencing TANs. Conclusion: CXCR2-inhibition induces reprogramming of the tumour immune microenvironment that promotes $\mathrm{ICI}$ in NASH-HCC.

\section{Significance of this study}

\section{What is already known on this subject?}

- Immune checkpoint inhibition therapy is emerging as a promising new therapy for the treatment of advanced hepatocellular carcinoma (HCC).

- Only a minority of HCC patients will respond to immune checkpoint inhibition (ICI) therapy and recent data suggest that $\mathrm{HCC}$ on the background of NASH may have reduced sensitivity to this treatment strategy.

- Neutrophils are a typical myeloid component of the liver in NASH and are found either within the HCC tumour microenvironment or in a peritumoural location.

- Neutrophils have considerable phenotypic plasticity and can exist in both tumour promoting and tumour suppressing states.

- Neutrophils may have the ability to influence ICI therapy. 


\section{What are the new findings?}

- $\mathrm{CXCR2}^{+}$neutrophils are found in human NASH and within the tumour of both human and mouse models of NASH-HCC.

- The resistance of NASH-HCC to anti-PD1 therapy is overcome by co-treatment with a CXCR2 small molecule inhibitor, with evidence of reduced tumour burden and extended survival.

- Anti-PD1 and CXCR2 inhibitor combine to selectively reprogramme tumour-associated neutrophils (TANs) from a pro- to an anti-tumour phenotype.

- Reprogrammed TANs proliferate locally within Granzyme $\mathrm{B}^{+}$immune clusters that contain physically associating $\mathrm{CD} 8^{+} \mathrm{T}$ cells and antigen presenting cells.

- Conventional XCR1 ${ }^{+}$dendritic cells (CDC1s) are found to be elevated in anti-PD1 and CXCR2 inhibitor treated $\mathrm{HCCs}$ and together with $\mathrm{CD} 8^{+} \mathrm{T}$ cells are required for therapeutic benefit.

\section{How might it impact on clinical practice in the foreseeable future?}

- TANs can be selectively manipulated to adopt an anti-tumour phenotype which unlocks their potential for cancer therapy. The ability of CXCR2 antagonism to combine with ICI therapy to bring about enhanced therapeutic benefit in NASH-HCC (and potentially in HCC of other aetiologies) warrents clinical investigation.

\section{Introduction}

Primary liver cancer is emerging globally as one of the most common and deadly malignancies with 905,000 new diagnosed cases and 830,000 deaths recorded in $2020^{1}$. Hepatocellular carcinoma (HCC) accounts for up to $85 \%$ of primary liver cancers and develops on the background of chronic liver disease (CLD) caused by persistent virological (HBV and HCV) or non-virological liver damage. Due to the increasing prevalence of obesity and the metabolic syndrome a high proportion of HCC is now attributed to non-alcoholic steatohepatitis (NASH), identified as the most common risk factor for HCC in UK and USA ${ }^{2,3}$.

Possible curative options for HCC such as tumour resection, liver transplant or ablation are at present limited to a minority of patients who are diagnosed at an early stage of the disease ${ }^{4}$. For more advanced HCC, approved systemic therapies include multikinase inhibitors and anti-VEGF agents. More recently, immune checkpoint inhibition (ICI) has emerged as a therapeutic modality in HCC with PD1 antibodies (nivolumab and pembrolizumab) being approved, and a combination of anti-PDL1 (atezolizumab) with anti-VEGF (bevacizumab) now being first-line treatment for advanced $\mathrm{HCC}^{5-7}$. However, only a minority (up to $30 \%$ ) of HCC patients respond to immunotherapy ${ }^{5-8}$. Moreover, it was recently reported that $\mathrm{HCC}$ on the background of NASH is less responsive to immunotherapy due to a 
$129 \mathrm{NASH}$-induced alteration in the immune components of the liver and in particular an expansion in 130 numbers of exhausted $\mathrm{CD} 8^{+} \mathrm{PD}^{+} \mathrm{T}$ cells, that appear to promote, rather than suppress, $\mathrm{NASH}-\mathrm{HCC}^{9,10}$. 131 Therefore, advanced therapeutic strategies for HCC will require a deeper appreciation of the complex 132 immune landscape of the tumour microenvironment, and in particular, should also take into account 133 the influence that the background liver pathology may have on the numbers, regional distributions, 134 phenotypes and activities of key immune cell types of relevance to cancer growth.

136 Recent use of imaging mass cytometry and single cell sequencing to probe the cellular constituents of 137 human HCC revealed considerable heterogeneity within the tumour microenvironment with 138 intratumoural region-specific distributions of immune cells ${ }^{11}$. Regions with evidence of less aggressive 139 cancer and ongoing liver damage (fibrogenesisis) were enriched for $\mathrm{CD}^{+} \mathrm{T}$ cells, $\mathrm{B}$ cells and

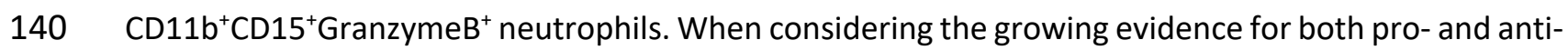
141 tumour functions for neutrophils in a variety of cancers ${ }^{12,13}$ including HCC $^{14}$ we were interested to 142 determine if modulation of neutrophil biology within the tumour microenvironment would influence 143 the resistance of NASH-HCC to anti-PD1 immunotherapy. Here, we determined that the CXC chemokine receptor CXCR2 is almost exclusively located on neutrophils in human and mouse NASH-HCC. This finding led us to ask if antagonism of CXCR2 can combine with anti-PD1 to overcome resistance of NASH-HCC to immunotherapy. Our findings suggest that this combination therapy reprogrammes the phenotype of tumour neutrophils and enhances their association with $\mathrm{CD}^{+} \mathrm{T}$ cells and conventional dendritic cells (CDC). Reshaping the tumour immune microenvironment was associated with a T cell- and DC-dependent reduction in tumour burden and increased survival. We propose that combined neutrophil phenotype modification and ICI may achieve improved outcomes in NASH-HCC.

154 Pro-tumour CXCR2 ${ }^{+}$neutrophils associate with NASH-HCC resistance to anti-PD1 immunotherapy To investigate the immunological determinants of unresponsiveness of NASH-HCC to anti-PD1 therapy we designed an orthotopic mouse model using the Hep-53.4 HCC cell line, which was selected due to

157 its high mutational burden (Supplementary Data Fig. 1a-c). On the background of steatosis induced 158 by a modified diet of high sugar and fat, we observed weight gain and larger tumours compared to 159 non-steatotic controls (Supplementary Data Fig. 1d-g). Tumours in non-steatotic controls were 160 responsive to anti-PD1 therapy, however, anti-PD1 showed no benefit on tumour burden, survival, 161 steatosis, proliferation or immune cell infiltration in steatotic mice (Fig. 1a-f and Supplementary Data 162 Fig. 1h-k). For an additional autochthonous model, we employed either Diethylnitrosamine (DEN) 
163

164

165

166

167

168

169

170

171

172

173

174

175

176

177

178

179

180

181

182

183

184

185

186

187

188

189

190

191

192

193

194

195

196

alone or in combination with the American lifestyle induced obesity syndrome diet (DEN/ALIOS), the latter to establish HCC on a background of $\mathrm{NASH}^{15,16}$ (Supplementary Data Fig. 11-o). Anti-PD1 responsiveness was observed in DEN mice fed a control diet, whereas anti-PD1 therapy had no effect on tumour burden, proliferation, or steatosis and animal survival when mice were fed the ALIOS diet

(Fig. 1g-I and Supplementary Data Fig. 1p, q). However, $\mathrm{F} 4 / 80^{+}$and $\mathrm{CD}^{+}$immune cell infiltrates were increased in anti-PD1 treated ALIOS fed mice (Supplementary Data Fig. 1r, s) indicative of anticipated alterations in tumour immunity.

Although elevated numbers of circulating neutrophils are associated with reduced $\mathrm{HCC}$ survival ${ }^{17}$, by contrast an enrichment of tumour associated neutrophils (TANs) is reported to correlate with improved survival ${ }^{18}$. This latter observation indicates a potential for TANs to influence the progression of HCC and raises the question of whether immunotherapy is influencing TANs (and vice versa). Ly6G ${ }^{+}$ neutrophils were found to be present in both tumour and non-tumour tissue of orthotopic-HCC mice and were significantly elevated in both compartments in the presence of NASH and remained high with anti-PD1 therapy (Fig 2a and Supplementary Data Fig. 2a). Increased numbers of TANs were also a feature in the DEN/ALIOS model and the increase reached significance with anti-PD1 treatment (Fig. 2b and Supplementary Data Fig. 2a). In addition, TANs were elevated in choline deficient-high fat diet (CD-HFD) spontaneous NASH-HCC model, and were retained with anti-PD1 therapy which is reported to also fail in this model ${ }^{9}$ (Supplementary Data Fig. 2b, c). Thus, we consistently observe TANs to accumulate in NASH-HCC, independent of the model examined, and they are retained in the tumour with anti-PD1 therapy. TANs display functional heterogeneity including anti- or pro-tumour phenotypes that impact on tumour growth ${ }^{19}$. Using transcriptomic profiling of tumour-isolated Ly $6 \mathrm{G}^{+}$ cells, we determined the phenotype of TANs from DEN/ALIOS tumours. To account for environmentally-induced differences in gene expression ${ }^{20}$, we compared TANs with peripheral blood and liver neutrophils. DEGs with increased expression were enriched for process networks associated with inflammatory (e.g. Nfkb1/Rel, Mapk8/Jnk1, Mapk9/Jnk2, Icam1) and calcium (e.g. Itpr1, Plcb1, Plcg1) signaling (Fig. 2c and Supplementary Data Fig. 2d). Genes associated with a pro-tumour neutrophil phenotype, including $C s f 1, C c / 3$, Vegfa and $P \operatorname{tgs} 2^{19-21}$ were also significantly up-regulated in TANs (Supplementary Data Fig. 2e).

Transcriptomic analysis of DEN/ALIOS tumours identified an up-regulation of myeloid associated cytokine and chemokine gene expression compared to normal liver (Fig. 2d). Notably, ligands (Cxcl1, $C x \mathrm{C} / 2, \mathrm{Cx} / 3, \mathrm{CxCl5})$ for the chemokine receptor $\mathrm{CXCR2}$, the latter identified as being pre-dominantly expressed by Ly6G $^{+}$neutrophils, were all increased in tumour tissue (Fig. 2d, e and Supplementary 
197 Data Fig. 2f). In situ hybridation analysis of $C x c r 2$ expression in DEN/ALIOS mouse tumours confirmed expression of $C_{x} \mathrm{x} 2$ to be specifically associated with morphologically identified infiltrating neutrophils and absent in parenchymal and tumour cells (Fig. 2f). This identifies CXCR2 as a neutrophil chemokine receptor that could be targeted to manipulate TANs in models of HCC-NASH ${ }^{14}$. In humans, the CXCR2 ligands CXCL1 and CXCL8 were significantly upregulated in NASH-HCC compared to NASH (Supplementary Data Fig. 2g). Neutrophil chemotaxis/migratory gene ontology (GO) terms were enriched in advanced human NASH (F4 fibrosis) and numbers of hepatic CD66 $\mathrm{b}^{+}$neutrophils increased with severity of NASH (Supplementary Data Fig. $\mathbf{2 h}$, i). Moreover, in HCC patient tissue, CD66b+ neutrophils and $\mathrm{CXCR2}^{+}$cells predominantly localised to NASH-HCC tumours with expression of the two markers correlating, and furthermore being demonstrated to be co-localised at the cellular level (Fig. $\mathbf{2 g}$, $\mathbf{h}$ and Supplementary Data Fig. $\mathbf{2 j}$, k). Similar to the mouse models, CXCR2 expression was limited to infiltrating immune cells and was absent on tumour epithelium within HCC in patients (Fig. 2i). We additionally noted that neutrophil expression signatures were enriched in human NASH-HCC compared to hepatitis B virus (HBV-HCC), hepatitis C virus (HCV-HCC) and alcohol-related-HCC ${ }^{22}$ (Fig. 2j). Thus, tumour infiltration of CXCR2-expressing neutrophils is characteristic of both murine models and human NASH-HCC and associates with resistance to anti-PD1 therapy in experimental models of NASH-HCC

\section{CXCR2 antagonism re-sensitises NASH-HCC to immunotherapy}

We next determined the effects of a CXCR2 small molecule inhibitor (AZD5069 ${ }^{23}$ ) in experimental NASH-HCC either administered alone or in combination with anti-PD1. We hypothesised that AZD5069 would suppress hepatic neutrophil recruitment. This was confirmed in the context of DEN-induced acute liver damage (Supplementary Data Fig. 3a-c). We also observed no change in $\mathrm{F} 4 / 80^{+}$ macrophages and $\mathrm{CD}^{+} \mathrm{T}$ cells (Supplementary Data Fig. 3d, e). These data are consistent with previous studies showing that in acute inflammatory settings CXCR2 inhibition selectively reduces neutrophil recruitment ${ }^{23}$.

Treatments using either or both AZD5069/anti-PD1 were then investigated for their ability to suppress tumour growth in the DEN-ALIOS model (Fig. 3a). Tumour burden at day 284 was reduced for AZD5069 monotherapy and with combined AZD5069/anti-PD1 treatment compared to vehicle and anti-PD1 monotherapy, however, no change in tumour number was identified suggesting a suppression of cancer progression (Fig. 3b and Supplementary Data Fig. 3f, g). Examination of tumours revealed reduced numbers of epithelial mitotic bodies and a lower tumour-stage grading for the AZD5069/anti- 
significantly altering the underlying NASH pathology (Fig. 3c-f and Supplementary Data Fig. 3h). This is clinically relevant as a high mitotic index in human HCC is a predictor of shorter disease-specific survival ${ }^{24}$. It was therefore noteworthy that the combination of AZD5069/anti-PD1 improved survival relative to monotherapies (Fig. 3g) Importantly, the benefits of AZD5069/anti-PD1 therapy were recapitulated in the orthotopic NASH-HCC model (Fig. $\mathbf{3 h}$-j). In contrast to the DEN/ALIOS model, a lack of therapeutic effect was observed with either AZD5069 or anti-PD1 monotherapy (Fig. 3i and

237 Supplementary Data Fig. 3j). However, AZD5069/anti-PD1 combination therapy reduced tumour 238 burden at day 28 and extended survival relative to vehicle control and monotherapies (Fig. $\mathbf{3 i}, \mathbf{j}$ and 239 Supplementary Data Fig. 3i). Notably, the treatments had no influence on steatosis or body weight 240 (Supplementary Data Fig. 3j, k). AZD5069/anti-PD1 treated mice reached clinical endpoint later, at 241 which point tumour burden was similar between treatment arms, this being consistent with 242 suppression of tumour growth (Supplementary Data Fig. 31). Hence, although CXCR2 antagonism 243 alone delivered modest model-dependant anti-tumour benefit, similar to observations made in 244 models of non-hepatic cancers $^{25-30}$, we show that CXCR2 inhibition sensitises to anti-PD1 immunotherapy in models of NASH-HCC that are otherwise resistant to anti-PD1 monotherapy.

\section{AZD5069/anti-PD1 therapy promotes an anti-tumour immune microenvironment.}

To further examine the concept that CXCR2 antagonism sensitises NASH-HCC to anti-PD1 therapy we asked if combination therapy activates classic T-cell mediated anti-tumour immunity. Characterisation of intratumoural T cells revealed intratumoural $\mathrm{CD} 8^{+} \mathrm{T}$ cells were significantly increased in both antiPD1 and AZD5069/anti-PD1 therapy groups, with only anti-PD1 monotherapy significantly affecting $\mathrm{CD}^{+} \mathrm{T}$ cells (Fig. 4a and Supplementary Data. Fig 4a). Combination therapy also enhanced intratumoural $\mathrm{CD}^{+} \mathrm{T}$ cell numbers in the orthotopic model (Supplementary Data Fig. 4b) Flow cytometric analysis revealed no gross phenotypic changes in early effector $\mathrm{CD} 8^{+} \mathrm{CD} 44^{\mathrm{Hi}} \mathrm{T}$ cells across treatment groups. However, anti-PD1 treatment alone significantly increased numbers of $C D 8^{+} P D 1^{+} T$ cells, this effect being recently reported by Pfister et $a l^{9}$ who suggested this $T$ cell phenotype compromises the efficacy of anti-PD1 treatment in NASH-HCC (Supplementary Data Fig. 4c). The percentage of $\mathrm{CD}^{+}{ }^{+} \mathrm{PD} 1^{+} \mathrm{T}$ cells was also higher in the context of anti-PD1 monotherapy relative to other treatment groups (Supplementary Data Fig. 4d). Upregulation of Granzyme K (Gzmk), and the T-box transcription factor Eomes combined with down-regulation of Tbx21 is associated with an "exhausted-like" pro-inflammatory $\mathrm{CD} 8^{+} \mathrm{PD} 1^{+} \mathrm{T}$ cell that has recently been reported to undergo clonal expansion during ageing ${ }^{31}$. Further investigation of T cell phenotype by RNAseq on isolated $\mathrm{CD}^{+}$cells revealed enhanced expression of Gzmk and Eomes following anti-PD1 therapy, both of which were 
increased with combination therapy (Fig. 4b). Alongside these changes, AZD5069/anti-PD1 therapy enhanced the expression of Granzyme B (Gzmb), a cytotoxic serine protease expressed by neutrophils, NK cells and by recently activated $\mathrm{CD} 8^{+} \mathrm{T}$ cells and for which expression correlates with clinical outcome in PD1 immunotherapy ${ }^{32-35}$. Immunostaining of DEN/ALIOS tumours revealed that Gzmb was detected at low levels in vehicle and monotherapy groups yet in the context of AZD5069/anti-PD1 combination therapy was highly expressed and was localised within discrete immune cell clusters containing banded immature neutrophils and lymphocytes (Fig. 4c and Supplementary Data Fig. 4e). Enhanced Gzmb protein expression was also achieved with combination therapy in orthotopic tumours where we also noted that anti-PD1 monotherapy depressed expression of the protease relative to vehicle control (Supplementary Data Fig. 4f). These data led us to ask if depletion of CD8 ${ }^{+}$ T cells would modulate the anti-tumour effects of AZD5069/anti-PD1 therapy. Depletion of CD8 ${ }^{+} \mathrm{T}$ cells was carried out by administration of anti-CD8 $\alpha$ to mice bearing an orthotopic NASH-HCC tumour and alongside AZD5069/anti-PD1 treatment (Fig. 4d). Succesful depletion of CD8 ${ }^{+} \mathrm{T}$ cells was confirmed by an increase in the proportion of $\mathrm{CD}^{+}$cells relative to the total $\mathrm{CD}^{+}$population (Supplementary Data Fig. 4g-i) and resulted in a higher orthotopic tumour burden compared with IgG controls (Fig. 4e). The requirement for $\mathrm{CD}^{+} \mathrm{T}$ cells for the anti-tumour effect of combinaton therapy was additionally confirmed by performing anti-CD $8 \alpha$-mediated depletion in tumour-bearing DEN/ALIOS mice treated with combination AZD5069/anti-PD1 (Supplementary Data Fig. 4j-m).

As recruitment and activation of XCR1 $1^{+}$conventional dendritic cells (CDC1) in tumours is considered critical for activation of cytotoxic $C D 8^{+} T$ cells and immunotherapy ${ }^{36}$ we next assessed CD86 surface expression as a marker of CDC1 activation in mice treated with AZD5069/anti-PD1 therapy. Anti-PD1 alone had no effect on activation of intratumour $X C R 1^{+}$CDC1 cells compared to vehicle controls in the DEN/ALIOS model (Fig. 4f). AZD5069 alone also had no effect on activation of intratumour XCR1 ${ }^{+}$CDC1 cells, likely due to the limited expression of CXCR2 on CDCs (Fig. 2e). However, combined AZD5069/anti-PD1 therapy substantially increased the activation of intratumoural cDC1 cells (Fig. 4f). As several CC chemokines associated with DC recruitment were expressed in mouse NASH-HCC tumours responding to mono and dual therapies (Supplementary Data Fig. 5a) we next determine the effects of perturbing DC recruitment employing mice that are deficient for $\operatorname{Ccr} 1, \mathrm{Ccr} 2, \mathrm{Ccr} 3$ and CCr5, termed iCCR ${ }^{37}$. These mice were treated as per the DEN/ALIOS model, and AZD5069/anti-PD1 or control therapy administered (Fig. $\mathbf{4 g}$ ). The number of CDC1 and CDC2 cells, and to a lesser extent $\mathrm{F} 4 / 80^{+}$macrophages but not neutrophils, were decreased in the tumours of iCCR mice (Supplementary Data Fig. 5b-d). Importantly, loss of myeloid recruitment alone in iCCR mice had no 
299 impact on tumour burden in the DEN/ALIOS model (Fig. 4h). However, unlike in wild-type (WT) mice, 300 AZD5069/anti-PD1 therapy failed to reduce tumour burden in iCCR mice (Fig. 4h). This loss of effect 301 was associated both with a reduction in tumour associated $\mathrm{CD}^{+} \mathrm{CD} 8^{+} \mathrm{T}$ cells and loss of granzyme $\mathrm{B}^{+}$ 302 immune clusters (Fig. $\mathbf{4} \mathbf{i}, \mathbf{j}$ ). To corroborate these data and to more specifically address the role of $303 \mathrm{XCR}^{+}$CDC1 cells we determined if AZD5069/anti-PD1 therapy of orthotopic NASH-HCC would be 304 affected by anti-XCL1-mediated blockade of XCL1, a major chemokine involved in mediating CDC1 and 305 CD8 T cell interactions (Fig. 4k) ${ }^{38}$. AZD5069/anti-PD1 therapy resulted in an increase in activated 306 intratumoural $X C R 1^{+}$CDC1 cells in line with observations in DEN/ALIOS mice, but with CDC1 activation 307 being selectively suppressed upon treatment with anti-XCL1 (Supplementary Data Fig. 5e, f). This 308 effect was associated with loss of the anti-tumoural action of AZD5069/anti-PD1 therapy; 309 demonstrated by increased tumour burden in anti-XCL1 treated mice compared to IgG controls (Fig.

310 4I). Confirming an associated impact on cytotoxic T cells, AZD5069/anti-PD1-induced increases in 311 tumoural cytotoxic $\mathrm{CD}^{+}$and $\mathrm{GzmB}^{+}$cells which was suppressed when $\mathrm{CDC} 1$ activation was selectively 312 blocked by anti-XCL1 (Fig. $4 \mathrm{~m}, \mathbf{n}$ ). We conclude that combined suppression of CXCR2 and PD1 313 stimulates both tumoural recruitment and activation of CDC1 cells enabling T cell-mediated 314 cytotoxicity.

AZD5069/anti-PD1 therapy promotes tumour neutrophil accumulation and the formation of intratumoural immunological hubs

318 Given that CXCR2 is almost exclusively expressed on neutrophils (Fig. 2e) we were curious as to their role in AZD5069/anti-PD1 therapy and its associated tumour immune cell remodelling. Unexpectedly, we observed that combination therapy in both models of NASH-HCC was associated with a dramatic increase in TANs, whereas AZD5069 monotherapy brought about the anticipated reduction in TANs

322 (Fig. 5a, b and Supplemental Data Fig. 6a). Real-time analysis of tumour neutrophil infiltration was not possible across the therapy time-course, so instead we examined circulating neutrophils sampled weekly from peripheral blood. Anti-PD1 alone had no demonstrable effects on circulating neutrophil numbers across the treatment period, whereas AZD5069 stimulated a transient increase in circulating Ly6G ${ }^{+}$neutrophils peaking at 4 weeks after start of treatment (Supplementary Data Fig. 6b). A similar transient increase in circulating neutrophils was observed in AZD5069/anti-PD1 treated mice, however, this effect was delayed peaking at 6 weeks from start of treatment. These peripheral blood data indicated a change in neutrophil behaviour in response to dual long-term targeting of CXCR2 and PD1. 
332 Immunohistochemical analysis of tumours identified clusters of TANs that were unique to 333 AZD5069/anti-PD1 treatment and comprising a mixed population of banded and segmented neutrophil populations (Fig. 5b, c and Supplementary Data Fig. 6c). The presence of these clustered TANs in AZD5069/anti-PD1 treated HCCs was intriguing and suggestive of local proliferation. Zhu et $a^{39}$ recently described early unipotent neutrophil progenitors (NeP) that produce neutrophils from adult BM. Conspicuously, NePs were significantly increased not only in the BM but also in tumours of AZD5069/anti-PD1 treated mice (Fig. 5d and Supplementary Data Fig. 6d,e). AZD5069/anti-PD1 treatment, therefore, alters granulopoiesis, while intratumour NePs may locally generate neutrophils, thus offering an explanation for the unexpectedly elevated numbers of TANs observed in mice receiving combination therapy.

To validate the presence of immature neutrophils in combined AZD5069/anti-PD1 treated tumours we utilised imaging mass cytometry (IMC) of tumour sections from DEN/ALIOS treatment arms (Supplementary Data Fig. 6f-j). Neutrophils, both immature and mature, were identified as expressing the primary granule protein MPO. We confirmed intratumoural clusters of proliferating $\mathrm{MPO}^{+} \mathrm{Ki} 67^{+}$ neutrophils to be significantly increased in AZD5069/anti-PD1 treated mice compared with monotherapies and vehicle controls (Fig. 5e, f). IMC neighbourhood analysis revealed intimate associations of $\mathrm{MPO}^{+} \mathrm{Ki} 67^{+}$neutrophils with $\mathrm{CD}^{+} \mathrm{T}$ cells and $\mathrm{MHC}$ Class $\mathrm{II}^{+}\left(\mathrm{MHCII}^{+}\right)$antigen presenting cells (APCs) that were found in the regions of interest with six out of seven AZD5069/anti-PD1 treated tumours that were examined by IMC (Fig. 5g-i). In contrast, for anti-PD1 and AZD5069 monotherapies, IMC only detected these mixed immune cell hubs in one tumour for each type of treatment (Fig. 5i). Intravital microscopy confirmed the presence of stable tumour-associated $\mathrm{Ly}_{6 \mathrm{G}}{ }^{+}$clusters, in vivo, in AZD5069/anti-PD1 treated mice (Supplementary Data Fig. 6k, I). Directly interacting Ly6G ${ }^{+}$TANs and $\mathrm{CD}^{+} \mathrm{CD} 8^{+} \mathrm{T}$ cells that maintained physical contact over several minutes or more were also observed (Supplementary Data Fig. 6m).

Longitudinal imaging of ex vivo precision cut liver slices (PCLS) was then performed to further interrogate $\mathrm{Ly}_{6 \mathrm{G}}{ }^{+}$cell (neutrophil), $\mathrm{CD}^{+}$cell ( $\mathrm{T}$ cell) and $\mathrm{CD} 11 \mathrm{c}^{+}$cell (DC and a subset of macrophages) dynamics within the tumours of DEN/ALIOS mice (Supplementary Data Fig. 6n, o, Supplementary Movie). PCLS from AZD5069/anti-PD1 treated mice had the expected, elevated numbers of neutrophils, CD11 $\mathrm{c}^{+}$cells and $\mathrm{CD} 8^{+} \mathrm{T}$ cells (Supplementary Data Fig. 6p-r). Although $\mathrm{T}$ cell speeds remained low in PCLS from all groups, neutrophil speed was increased in AZD5069/anti-PD1 treated tumours suggesting a more actively migrating phenotype for these neutrophils (Supplementary Data

365 Fig. 6s, t). Neutrophil-CD11 ${ }^{+}$cell interactions were high in tumours irrespective of treatment, 
however, neutrophil-CD8 ${ }^{+} \mathrm{T}$ cell and $\mathrm{CD} 11 \mathrm{C}^{+}-\mathrm{CD} 8^{+} \mathrm{T}$ cell interactions were elevated in AZD5069/antiPD1 treated tumours compared to vehicle controls (Supplementary Data Fig. 6u-y, Supplementary Movie). These data provide evidence that combined therapeutic targeting of $\mathrm{CXCR2}^{+}$neutrophils and the PD1-PDL1 immune checkpoint remodels the NASH-HCC tumour immune microenvironment, including the generation of locally proliferating immature neutrophil progenitors in close physical association with cytotoxic T cells.

372

AZD5069/anti-PD1 combination therapy re-programmes the TAN phenotype.

374 Given that our observations were consistent with intratumoural granulopoiesis in response to combination AZD5069/anti-PD1 therapy, we more closely characterised the TAN phenotype under these conditions. Grieshaber-Bouyer et $a l^{40}$, recently reported a chronologically ordered developmental path for neutrophils termed 'neutrotime'. This extends from immature preneutrophils (early neutrotime) that are predominantly found in bone marrow (BM) to fully mature neutrophils (late neutrotime) mainly located in the circulation and spleen (Supplemental Data Fig. 7a). TAN transcriptome analysis revealed that AZD5069/anti-PD1 therapy induced neutrotime reprogramming along this neutrotime spectrum (Fig. 6a and Supplementary Data Fig. 7b). TANs in vehicle, anti-PD1 and AZD5069 treated tumours phenotypically resembled mature neutrophils, expressing genes characteristic of the late neutrotime (e.g. Jund, Csf3r, Rps27) (Fig. 6a and Supplementary Data Fig. 7b). However, late neutrotime genes were comprehensively downregulated in TANs from AZD5069/anti-PD1 treated mice, with a corresponding upregulation of transcripts characteristic of the early neutrotime (e.g. Mmp8, Retnlg, Ltf, Lcn2, Camp, Chil3, Tuba1b, Fcnb). Lactoferrin (Ltf) was of particular interest amongst the early neutrotime genes as its protein has well documented anti-cancer activities; including the activation of DCs and macrophages and enhancing the cytotoxic properties of natural killer cells ${ }^{41-43}$. Staining for Lactoferrin in DEN/ALIOS tumours was elevated in AZD5069/anti-PD1 treated mice where the protein was localised to the neutrophil-rich immune clusters that included banded immature neutrophils (Fig. 6b,c). These observations indicate a potential mechanism by which reprogrammed TANs may network with other immune cells to enact anti-tumoural effects. Interrogation of transcriptome data from NASH- and non-NASH-related HCC patients identified the late neutrotime signature to be significantly enriched in NASH-HCC when compared to HCCs of other aetiologies $^{44}$ (Fig. 6d). Moreover, the late neutrotime profile was specifically associated with human HCCs that are stratified by gene expression to the immune class and specifically within this group to the exhausted immune class which are typically resistant to immunotherapy (Fig. 6e ${ }^{44-47}$. This 
suggests that TANs in human NASH-HCC resemble the mature phenotype of TANs in mouse NASH$\mathrm{HCC}$ and may play a role in preventing $\mathrm{ICl}$ responses in patients, and as such we speculate they may be susceptible to similar therapeutic neutrotime reprogramming with AZD5069/anti-PD1 treatment. To examine the stage at which neutrophils are reprogrammed we compared tumoural to circulating neutrophil profiles in the treatment groups in the DEN/ALIOS model. Neutrotime reprogramming was specific to the intratumoural population of AZD5069/anti-PD1 treated mice and noteably without an associated neutrotime change in circulating neutrophils of this treatment group (Fig. 6f), this observation being consistent with tumour-selective neutrophil reprogramming. Hence, while the combination therapy brings about reprogramming of TAN maturity, it leaves intact the mature phenotype of circulating neutrophils required for their classic anti-microbial surveillance functions $\mathbf{s}^{48,49}$.

410

RNA-Seq of purified Ly6G ${ }^{+}$neutrophils revealed that TANs from AZD5069/anti-PD1 treatment mice were enriched for process networks associated with cell cycle, phagocytosis and antigen presentation when compared to vehicle controls (Fig. 6g and Supplementary Data Fig. 7c). AZD5069 monotherapy modestly enhanced the expression of signatures associated with cell division, phagocytosis, and degranulation while also eliciting a reduction in pro-tumour gene expression, with all of these effects being accentuated when AZD5069 was combined with anti-PD1 (Fig. 6g and Supplementary Data Fig. 7c-e). Anti-PD1 treatment promoted antigen presentation and processing signatures, which were also enriched in combined AZD5069/anti-PD1 treatment but not with AZD5069 monotherapy (Fig. 6g). These findings were again indicative of the combinatorial effects of AZD5069/anti-PD1 therapy on TAN phenotype. AZD5069 monotherapy (but not anti-PD1 monotherapy) suppressed the expression of key immune checkpoint molecules in TANs, including downregulation of Cd80, Pvr, Sirpa, Pdl1 and PdI2.

422 This loss of immune checkpoint gene expression was maintained in the context of combination 423 therapy and for some genes (e.g. Pvr and Srpa) we noted more pronounced suppressive effects when 424 compared to the AZD5069 montherapy alone (Supplementary Data Fig. 7f). Hence, TAN-enriched immune hubs observed in AZD5069/anti-PD1 treated tumours are able to avoid immune checkpoint inhibition signals that might otherwise cause immune exhaustion. AZD5069/anti-PD1 TANs also displayed a strong correlation with transcriptional changes seen in neutrophils during an acute systemic inflammatory response ${ }^{50}$, including expression of genes involved in exocytosis, myeloid cell activation and degranulation (Supplementary Data Fig. 7g, h). Finally, these AZD5069/anti-PD1 TANs closely resembled a recently objectively characterised acute-inflammatory immature-Ly6G ${ }^{\ln t}$ neutrophil population isolated from lipopolysaccharide-(LPS)-treated mice ${ }^{50}$ (Supplementary Data

432 Fig. 7i). In summary, AZD5069/anti-PD1 combination therapy brings about reprogramming of HCC433 NASH TANs to exhibit immature, proliferative and inflammatory characteristics. 
435 From these data we hypothesised that activated early neutrotime TANs have anti-tumoural properties. Due to their relatively low numbers and lack of specific surface markers it was not possible to isolate reprogrammed TANs from tumours in order to formally test this hypothesis. Instead, as proof-of-principe, we isolated inflammatory immature neutrophils enriched in the bone marrow of LPS-treated mice and a pool of mature bone marrow neutrophils isolated from control PBS treated mice. Adoptively transferring these cells to orthotopic NASH-HCC mice, we asked whether they would bring about an anti-tumoural effect in combination with anti-PD1 treatment (Fig. $\mathbf{6 h}$ and

442 Supplementary Data Fig. 7j, k). Transfer of inflammatory immature neutrophils lead to a significant 443 increase in circulating immature CXCR2 $^{\text {Lo }}$ neutrophils in the blood and resulted in a significant 444 reduction in tumour burden (Fig. 6i and Supplementary Data Fig. 7l). In contrast transfer of mature neutrophils had no effect on tumour burden (Fig. 6i). To investigate underlying mechanism we examined intratumoural $\mathrm{CDC1}$ and $\mathrm{CD} 8^{+} \mathrm{T}$ cells. Similar to treatment of mice with AZD5069/anti-PD1, we noted transfused immature neutrophils caused increased activation $\left(C D 86^{+}\right)$of intratumoural $\mathrm{XCR} 1^{+} \mathrm{CDC} 1$ cells and elevated $\mathrm{CD} 8^{+} \mathrm{T}$ cells in tumours, unlike mice transfused with equal numbers of mature neutrophils (Fig. 6j-I). Moreover, the adoptive transfer of neutrophils from LPS treated was associated with increased intratumoural Gzmb expression indicative of stimulation of cytotoxic activity within the tumour (Fig. $6 \mathrm{~m}$ ). Hence, we conclude that bone-marrow derived immature inflammatory neutrophils which have phenotypic similarities to AZD5069/anti-PD1 reprogrammed TANs are able to stimulate immune remodelling within HCC tumours and promote anti-tumoural effects.

\section{Discussion}

457 Immune-based therapies hold considerable promise for the treatment of advanced HCC, however at present response rates are low and according to recent reports this is at least in-part determined by the immune cell composition of the tumour ${ }^{45,51,52}$. HCC on the background of NASH presents additional considerations because of the crosstalk occurring between inflammatory cells and various metabolic adaptions manifest in the disease such as insulin resistance, steatosis, oxidative stress and altered mitochondrial function ${ }^{53}$. Pfister and colleagues have reported that immunotherapy in NASH-HCC may

463 be compromised due to high numbers of pro-tumour $\mathrm{CD} 8^{+} \mathrm{PD} 1^{+} \mathrm{T}$ cells in the tumour 464 microenvironment ${ }^{9}$. Here we show that selective targeting of neutrophils with a CXCR2 antagonist 465 promotes the anti-tumour effects of anti-PD1 therapy in NASH-HCC, this effect being mechanistically 466 associated with activation of classic $C D 8^{+} T$ cell and DC mediated anti-tumour immunity, but also with 467 intratumoural reprogramming of TAN maturation and phenotype. Based on imaging mass cytometry 
we propose that the reprogrammed TANs, which are characterised by their proliferative and inflammatory characteristics, associate in tight clusters with $\mathrm{CD} 8^{+} \mathrm{T}$ cells and APCs to form anti-tumour Gzmb-secreting immune hubs within the NASH-HCC tumour microenvironment. Our work therefore emphasises the strong potential for targeted therapeutic manipulation of the innate immune system in cancer, but also uncovers a previously unrecognised crosstalk between the C-X-C chemokine/CXCR2 and PD1/PDL1 signaling systems that may be exploited to improve immunotherapy responses not only in NASH-HCC but also in other types of cancer that exhibit immunotherapy resistance ${ }^{54,55}$.

476 Neutrophil infiltration is a key pathological feature of human NASH that may result from upregulation 477 of hepatic CXCL8 (IL-8) and CXCL1 ${ }^{56,57}$, which we also report here to be enriched in human NASH-HCC. 478 In addition, expression of CXCR2 on neutrophils in NASH is selectively enhanced through an auto479 stimulation mechanism involving the upregulation of neutrophil-derived lipocalin $2^{58}$. Once present in 480 the NASH and NASH-HCC microenvironments neutrophils are exposed to high levels of TGF- $\beta$ which, as reported with other cancers ${ }^{19-21}$, can polarise TANs towards a so-called 'N2' tumour-promoting state $^{14}$. It is also pertinent to address the relationship between TANs and myeloid-derived suppressor cells (MDSC), the latter being a heterogenous population comprising polymorphonuclear granulocytic Ly6G $^{+}$Ly6C ${ }^{\text {Lo }}$ (PMN-MDSC) and monocytic Ly6G-Ly6C ${ }^{\mathrm{Hi}}$ (M-MDSC) cells. Accumulating evidence suggests that PMN-MDSC are immunosuppressive neutrophils and may be functionally very similar to the TANs that have been termed ' $N 2$ ', with shared pro-tumour properties ${ }^{14,59}$. In the mouse there are no markers to distinguish between PMN-MDSCs and neutrophils and as such we cannot rule out that TANs in mouse models of NASH-HCC include PMN-MDSCs which may also be susceptible to reprogramming in response to combined CXCR2 antagonism and anti-PD1 therapy. However, the typical inhibitory effects on $\mathrm{DC}$ and $\mathrm{CD} 8^{+} \mathrm{T}$ cell functions associated with the activities of PMN-MDSCs and immunosuppressive neutrophils were clearly overcome by combined AZD5069/anti-PD1 therapy. human cancers including; pancreatic, lung, ovarian, prostate, colon and now the liver ${ }^{25-29,60}$. Furthermore, in genetic murine models of lung cancer, inhibition of CXCR1 and 2 receptors in combination with anti-PD1 amplified anti-tumour responses ${ }^{61,62}$. The proposed mechanism of action, until now, however, was thought to rely on reprogramming of the tumour immune microenvironment, primarily as a result of impaired myeloid recruitment. The most remarkable immunobiological finding of our study was that, paradoxically, when combined with anti-PD1, CXCR2 inhibition leads to an

500 increase in liver neutrophils and a selective reprogramming of the TAN neutrotime, with no evidence 
502

503

504

505

506

507

508

509

510

511

512

513

514

515

516

517

518

519

520

521

522

523

524

525

526

527

528

529

530

531

532

533

534

535

reprogrammed TANs evokes extramedullary granulopoiesis which can be seen in mice following antibody-mediated depletion of $\mathrm{Ly}_{6 \mathrm{G}}{ }^{+}$cells and that is due to survival and expansion of residual tissue neutrophils driven by high systemic levels of granulocyte colony-stimulating factor ${ }^{63}$, indeed this rebound effect meant that we were unable to exploit this protocol to directly interrogate the function of reprogrammed TANs. However, as proof-of-principle we were able to establish that adoptive transfer of immature activated neutrophils isolated from bone marrow of LPS-treated mice has antitumour activity in NASH-HCC and this effect was accompanied by remodelling of tumour immunity including the activation of $\mathrm{CDC} 1$ cells, elevated $\mathrm{CD} 8^{+} \mathrm{T}$ cell counts and induction of anti-tumoural Gzmb; these being changes that were also noted with AZD506/anti-PD1 therapy. In future work it will be important to identify selective markers of the reprogrammed TANs that might be exploited for detailed functional characterisation, as well as for enabling their selective experimental manipulation which at present is not possible. Also, it will be important to determine precisely how and why combined CXCR2 antagonism and anti-PD1 treatment selectively induces proliferative immature neutrophils in the tumour. Clinically the ability to selectively reprogramme TANs while retaining mature anti-microbial neutrophils in the circulation may be very relevant in HCC since bacterial infections and septic shock are common clinical challenges in cirrhotic patients (in whom $90 \%$ of HCC develops $)^{64}$.

In summary, we present a novel combination immunotherapy that enhances the efficacy of anti-PD1 in NASH-HCC. As the CXCR2 antagonist AZD5069 has been demonstrated to be safe for use in humans it is timely to determine if HCC patients would benefit from a similar therapy.

\section{References}

1. Sung H, Ferlay J, Siegel RL, et al. Global Cancer Statistics 2020: GLOBOCAN Estimates of Incidence and Mortality Worldwide for 36 Cancers in 185 Countries. CA Cancer J Clin. 2021;71(3):209-249. doi:10.3322/caac.21660

2. Younossi Z, Stepanova M, Ong JP, et al. Nonalcoholic Steatohepatitis Is the Fastest Growing Cause of Hepatocellular Carcinoma in Liver Transplant Candidates. Clin Gastroenterol Hepatol. 2019;17(4):748-755.e3. doi:10.1016/j.cgh.2018.05.057

3. Dyson J, Jaques B, Chattopadyhay D, et al. Hepatocellular cancer: the impact of obesity, type 2 diabetes and a multidisciplinary team. J Hepatol. 2014;60(1):110-117.

$$
\text { doi:10.1016/j.jhep.2013.08.011 }
$$

4. Gallage S, García-Beccaria M, Szydlowska M, et al. The therapeutic landscape of hepatocellular carcinoma. Med. 2021;2:505-552. doi:10.1016/j.medj.2021.03.002 
536 5. Finn RS, Qin S, Ikeda M, et al. Atezolizumab plus Bevacizumab in Unresectable Hepatocellular Carcinoma. N Eng/ J Med. 2020;382(20):1894-1905. doi:10.1056/NEJMoa1915745

538

6. Yau T, Kang Y-K, Kim T-Y, et al. Efficacy and Safety of Nivolumab Plus Ipilimumab in Patients With Advanced Hepatocellular Carcinoma Previously Treated With Sorafenib. JAMA Oncol. 2020;6(11):e204564. doi:10.1001/jamaoncol.2020.4564

541 7. Finn RS, Ryoo B-Y, Merle P, et al. Pembrolizumab As Second-Line Therapy in Patients With Advanced Hepatocellular Carcinoma in KEYNOTE-240: A Randomized, Double-Blind, Phase III Trial. J Clin Oncol. 2020;38(3):193-202. doi:10.1200/JCO.19.01307

544 8. Yau T, Park J-W, Finn RS, et al. Nivolumab versus sorafenib in advanced hepatocellular carcinoma (CheckMate 459): a randomised, multicentre, open-label, phase 3 trial. Lancet Oncol. 2022;23(1):77-90. doi:10.1016/S1470-2045(21)00604-5

547 9. Pfister D, Núñez NG, Pinyol R, et al. NASH limits anti-tumour surveillance in immunotherapytreated HCC. Nature. 2021;592(7854):450-456. doi:10.1038/s41586-021-03362-0

10. Haber PK, Puigvehí M, Castet F, et al. Evidence-Based Management of Hepatocellular Carcinoma: Systematic Review and Meta-analysis of Randomized Controlled Trials (20022020). Gastroenterology. 2021;161(3):879-898. doi:10.1053/j.gastro.2021.06.008

11. Sheng J, Zhang J, Wang L, et al. Topological analysis of hepatocellular carcinoma tumour microenvironment based on imaging mass cytometry reveals cellular neighbourhood regulated reversely by macrophages with different ontogeny. Gut. Published online July 12 , 2021. doi:10.1136/gutjnl-2021-324339

12. Shaul ME, Fridlender ZG. Tumour-associated neutrophils in patients with cancer. Nat Rev Clin Oncol. 2019;16(10):601-620. doi:10.1038/s41571-019-0222-4

13. Coffelt SB, Wellenstein MD, de Visser KE. Neutrophils in cancer: neutral no more. Nat Rev Cancer. 2016;16(7):431-446. doi:10.1038/nrc.2016.52

560 14. Geh D, Leslie J, Rumney R, Reeves HL, Bird TG, Mann DA. Neutrophils as potential therapeutic targets in hepatocellular carcinoma. Nat Rev Gastroenterol Hepatol. Published online January 12, 2022:1-17. doi:10.1038/s41575-021-00568-5

15. Tetri LH, Basaranoglu M, Brunt EM, Yerian LM, Neuschwander-Tetri BA. Severe NAFLD with hepatic necroinflammatory changes in mice fed trans fats and a high-fructose corn syrup equivalent. Am J Physiol Gastrointest Liver Physiol. 2008;295:987-995. doi:10.1152/ajpgi.90272.2008.-The cancer in the absence of cirrhosis. Sci Rep. 2021;11(1):16727. doi:10.1038/s41598-02196076-2 
570 17. Margetts J, Ogle LF, Chan SL, et al. Neutrophils: driving progression and poor prognosis in hepatocellular carcinoma? Br J Cancer. 2018;118(2):248-257. doi:10.1038/bjc.2017.386

18. Esteban-Fabró R, Willoughby CE, Piqué-Gili M, et al. Cabozantinib enhances the efficacy and immune modulatory activity of anti-PD1 therapy in a syngeneic mouse model of hepatocellular carcinoma. J Hepatol. 2020;73:S40. doi:10.1016/S0168-8278(20)30632-2

19. Shaul ME, Levy L, Sun J, et al. Tumor-associated neutrophils display a distinct N1 profile following TGF $\beta$ modulation: A transcriptomics analysis of pro- vs. antitumor TANs. Oncoimmunology. 2016;5(11):e1232221. doi:10.1080/2162402X.2016.1232221

20. Fridlender ZG, Albelda SM. Tumor-associated neutrophils: friend or foe? Carcinogenesis. 2012;33(5):949-955. doi:10.1093/carcin/bgs123

580

21. Fridlender ZG, Sun J, Kim S, et al. Polarization of tumor-associated neutrophil phenotype by TGF-beta: \&quot;N1\&quot; versus \&quot;N2\&quot; TAN. Cancer Cell. 2009;16(3):183-194. doi:10.1016/j.ccr.2009.06.017

22. Pinyol R, Torrecilla S, Wang $\mathrm{H}$, et al. Molecular characterisation of hepatocellular carcinoma in patients with non-alcoholic steatohepatitis. J Hepatol. 2021;75(4):865-878. doi:10.1016/j.jhep.2021.04.049

23. Jamieson $\mathrm{T}$, Clarke $\mathrm{M}$, Steele $\mathrm{CW}$, et al. Inhibition of CXCR2 profoundly suppresses inflammation-driven and spontaneous tumorigenesis. J Clin Invest. 2012;122(9):3127-3144. doi:10.1172/JCI61067

24. Ha SY, Choi M, Lee T, Park C-K. The Prognostic Role of Mitotic Index in Hepatocellular Carcinoma Patients after Curative Hepatectomy. Cancer Res Treat. 2016;48(1):180-189. doi:10.4143/crt.2014.321

25. Yang G, Rosen DG, Liu G, et al. CXCR2 promotes ovarian cancer growth through dysregulated cell cycle, diminished apoptosis, and enhanced angiogenesis. Clin Cancer Res. 2010;16(15):3875-3886. doi:10.1158/1078-0432.CCR-10-0483

26. Steele CW, Karim SA, Leach JDG, et al. CXCR2 Inhibition Profoundly Suppresses Metastases and Augments Immunotherapy in Pancreatic Ductal Adenocarcinoma. Cancer Cell. 2016;29(6):832-845. doi:10.1016/j.ccell.2016.04.014 promotes therapeutic effect of cisplatin. Mol Cancer. 2021;20(1):62. doi:10.1186/s12943021-01355-1 
604

605

606

607

608

609

610

611

612

613

614

615

616

617

618

619

620

621

622

623

624

625

626

627

628

629

630

631

632

633

634

635

636

637

29. Katoh H, Wang D, Daikoku T, Sun H, Dey SK, Dubois RN. CXCR2-expressing myeloid-derived suppressor cells are essential to promote colitis-associated tumorigenesis. Cancer Cell. 2013;24(5):631-644. doi:10.1016/j.ccr.2013.10.009

30. Tang KH, Li S, Khodadadi-Jamayran A, et al. Combined Inhibition of SHP2 and CXCR1/2 Promotes Antitumor T-cell Response in NSCLC. Cancer Discov. 2022;12(1):47-61. doi:10.1158/2159-8290.CD-21-0369

31. Mogilenko DA, Shpynov O, Andhey PS, et al. Comprehensive Profiling of an Aging Immune System Reveals Clonal GZMK+ CD8+ T Cells as Conserved Hallmark of Inflammaging. Immunity. 2021;54(1):99-115.e12. doi:10.1016/j.immuni.2020.11.005

32. Hurkmans DP, Basak EA, Schepers N, et al. Granzyme B is correlated with clinical outcome after PD-1 blockade in patients with stage IV non-small-cell lung cancer. J Immunother cancer. 2020;8(1). doi:10.1136/jitc-2020-000586

33. Rousalova I, Krepela E. Granzyme B-induced apoptosis in cancer cells and its regulation (review). Int J Oncol. 2010;37(6):1361-1378. doi:10.3892/ijo_00000788

34. Wagner C, Iking-Konert C, Denefleh B, Stegmaier S, Hug F, Hänsch GM. Granzyme B and perforin: constitutive expression in human polymorphonuclear neutrophils. Blood. 2004;103(3):1099-1104. doi:10.1182/blood-2003-04-1069

35. Nowacki TM, Kuerten S, Zhang W, et al. Granzyme B production distinguishes recently activated CD8(+) memory cells from resting memory cells. Cell Immunol. 2007;247(1):36-48. doi:10.1016/j.cellimm.2007.07.004

36. Gardner A, de Mingo Pulido Á, Ruffell B. Dendritic Cells and Their Role in Immunotherapy. Front Immunol. 2020;11:924. doi:10.3389/fimmu.2020.00924

37. Dyer DP, Medina-Ruiz L, Bartolini R, et al. Chemokine Receptor Redundancy and Specificity Are Context Dependent. Immunity. 2019;50(2):378-389.e5.

doi:10.1016/j.immuni.2019.01.009

38. Deczkowska A, David E, Ramadori P, et al. XCR1+ type 1 conventional dendritic cells drive liver pathology in non-alcoholic steatohepatitis. Nat Med. 2021;27(6):1043-1054.

doi:10.1038/s41591-021-01344-3

39. Zhu YP, Padgett L, Dinh HQ, et al. Identification of an Early Unipotent Neutrophil Progenitor with Pro-tumoral Activity in Mouse and Human Bone Marrow. Cell Rep. 2018;24(9):23292341.e8. doi:10.1016/j.celrep.2018.07.097

40. Grieshaber-Bouyer R, Radtke FA, Cunin P, et al. The neutrotime transcriptional signature defines a single continuum of neutrophils across biological compartments. Nat Commun. 2021;12(1):2856. doi:10.1038/s41467-021-22973-9 
638

639

640

641

642

643

644

645

646

647

648

649

650

651

652

653

654

655

656

657

658

659

660

661

662

663

664

665

666

667

668

669

670

671

41. Actor JK, Hwang S-A, Kruzel ML. Lactoferrin as a natural immune modulator. Curr Pharm Des. 2009;15(17):1956-1973. doi:10.2174/138161209788453202

42. Spadaro $\mathrm{M}$, Montone $\mathrm{M}$, Arigoni $\mathrm{M}$, et al. Recombinant human lactoferrin induces human and mouse dendritic cell maturation via Toll-like receptors 2 and 4. FASEB J. 2014;28(1):416-429. doi:10.1096/fj.13-229591

43. Cutone A, Rosa L, laniro G, et al. Lactoferrin's Anti-Cancer Properties: Safety, Selectivity, and Wide Range of Action. Biomolecules. 2020;10(3). doi:10.3390/biom10030456

44. Villanueva A, Portela A, Sayols S, et al. DNA methylation-based prognosis and epidrivers in hepatocellular carcinoma. Hepatology. 2015;61(6):1945-1956. doi:10.1002/hep.27732

45. Sia D, Jiao Y, Martinez-Quetglas I, et al. Identification of an Immune-specific Class of Hepatocellular Carcinoma, Based on Molecular Features. Gastroenterology. 2017;153(3):812826. doi:10.1053/j.gastro.2017.06.007

46. Ayers $M$, Lunceford J, Nebozhyn $M$, et al. IFN- $\gamma$-related mRNA profile predicts clinical response to PD-1 blockade. J Clin Invest. 2017;127(8):2930-2940. doi:10.1172/JCI91190

47. Sangro B, Melero I, Wadhawan S, et al. Association of inflammatory biomarkers with clinical outcomes in nivolumab-treated patients with advanced hepatocellular carcinoma. J Hepatol. 2020;73(6):1460-1469. doi:10.1016/j.jhep.2020.07.026

48. Mayadas TN, Cullere X, Lowell CA. The Multifaceted Functions of Neutrophils. Annu Rev Pathol Mech Dis. 2014;9(1):181-218. doi:10.1146/annurev-pathol-020712-164023

49. Amulic B, Cazalet C, Hayes GL, Metzler KD, Zychlinsky A. Neutrophil Function: From Mechanisms to Disease. Annu Rev Immunol. 2012;30(1):459-489. doi:10.1146/annurevimmunol-020711-074942

50. Mackey JBG, McFarlane AJ, Jamieson T, et al. Maturation, developmental site, and pathology dictate murine neutrophil function. bioRxiv. Published online July 28 , 2021:2021.07.21.453108. doi:10.1101/2021.07.21.453108

51. Kurebayashi $\mathrm{Y}, \mathrm{Ojima} \mathrm{H}$, Tsujikawa $\mathrm{H}$, et al. Landscape of immune microenvironment in hepatocellular carcinoma and its additional impact on histological and molecular classification. Hepatology. 2018;68(3):1025-1041. doi:10.1002/hep.29904

52. Yarchoan $M$, Xing $D$, Luan $L$, et al. Characterization of the Immune Microenvironment in Hepatocellular Carcinoma. Clin Cancer Res. 2017;23(23):7333-7339. doi:10.1158/10780432.CCR-17-0950

53. Anstee QM, Reeves HL, Kotsiliti E, Govaere O, Heikenwalder M. From NASH to HCC: current concepts and future challenges. Nat Rev Gastroenterol Hepatol. 2019;16(7):411-428. doi:10.1038/s41575-019-0145-7 
54. Boi SK, Orlandella RM, Gibson JT, et al. Obesity diminishes response to PD-1-based immunotherapies in renal cancer. J Immunother cancer. 2020;8(2):e000725. doi:10.1136/jitc2020-000725

55. An Y, Wu Z, Wang N, et al. Association between body mass index and survival outcomes for cancer patients treated with immune checkpoint inhibitors: a systematic review and metaanalysis. J Transl Med. 2020;18(1):235. doi:10.1186/s12967-020-02404-x

56. Pan X, Chiwanda Kaminga A, Liu A, Wen SW, Chen J, Luo J. Chemokines in Non-alcoholic Fatty Liver Disease: A Systematic Review and Network Meta-Analysis. Front Immunol. 2020;11:1802. doi:10.3389/fimmu.2020.01802

57. Liu K, Wang F-S, Xu R. Neutrophils in liver diseases: pathogenesis and therapeutic targets. Cell Mol Immunol. 2021;18(1):38-44. doi:10.1038/s41423-020-00560-0

58. Ye D, Yang K, Zang S, et al. Lipocalin-2 mediates non-alcoholic steatohepatitis by promoting neutrophil-macrophage crosstalk via the induction of CXCR2. J Hepatol. 2016;65(5):988-997. doi:10.1016/j.jhep.2016.05.041

59. Zhou J, Nefedova Y, Lei A, Gabrilovich D. Neutrophils and PMN-MDSC: Their biological role and interaction with stromal cells. Semin Immunol. 2018;35:19-28. doi:10.1016/j.smim.2017.12.004

60. Tang KH, Li S, Khodadadi-Jamayran A, et al. Combined Inhibition of SHP2 and CXCR1/2 Promotes Anti-Tumor T Cell Response in NSCLC. Cancer Discov. Published online August 5, 2021. doi:10.1158/2159-8290.CD-21-0369

61. Kargl J, Zhu X, Zhang H, et al. Neutrophil content predicts lymphocyte depletion and anti-PD1 treatment failure in NSCLC. JCl insight. 2019;4(24). doi:10.1172/jci.insight.130850

62. Sun L, Clavijo PE, Robbins $\mathrm{Y}$, et al. Inhibiting myeloid-derived suppressor cell trafficking enhances T cell immunotherapy. JCl insight. 2019;4(7). doi:10.1172/jci.insight.126853

63. Moses K, Klein JC, Männ L, Klingberg A, Gunzer M, Brandau S. Survival of residual neutrophils and accelerated myelopoiesis limit the efficacy of antibody-mediated depletion of Ly-6G+ cells in tumor-bearing mice. J Leukoc Biol. 2016;99(6):811-823. doi:10.1189/jlb.1HI0715-289R

64. Bajaj JS, Kamath PS, Reddy KR. The Evolving Challenge of Infections in Cirrhosis. Longo DL, ed. N Engl J Med. 2021;384(24):2317-2330. doi:10.1056/NEJMra2021808

65. McCowan J, Fercoq F, Kirkwood PM, et al. The transcription factor EGR2 is indispensable for tissue-specific imprinting of alveolar macrophages in health and tissue repair. Sci Immunol. 2021;6(65):eabj2132. doi:10.1126/sciimmunol.abj2132 
Methods

707

708

Mice, diets and treatments

709

All animal experiments using the orthotopic NASH-HCC model and DEN/ALIOS model were performed

710 in accordance with a UK Home Office licence (PP8854860, PP390857 and PP0604995), adhered to ARRIVE guidelines (https://www.nc3rs.org.uk/arrive-guidelines), and in accordance with the UK Animal (Scientific Procedures) Act 1986, and were subject to review by the animal welfare and ethical review board of the University of Glasgow and Newcastle University. All mice were housed in specific pathogen free conditions with unrestricted access to food and water and maintained on a constant $12 \mathrm{hr}$ light-dark cycle under controlled climate $\left(19-22^{\circ} \mathrm{C}, 45-65 \%\right.$ humidity). All animal experiments using the CD-HFD were performed in accordance with German law and the governmental bodies, and with approval from the Regierungspräsidium Karlsruhe (G11/16, G129/16, G7/17). Male mice were housed at the German Cancer Research Center (DKFZ) (constant temperature of $20-24{ }^{\circ} \mathrm{C}$ and $45-65 \%$ humidity with a $12 \mathrm{hr}$ light-dark cycle and were maintained under specific pathogen-free conditions.

For the orthotopic NASH-HCC model, intrahepatic injection of Hep-53.4 cells into the left lobe of C57BL/6 mice was performed under isoflurane general anaesthesia. Mice were fed ad libitum either a normal chow diet plus drinking water or a modified western diet (Envigo -TD.120528) plus sugar water ( $23.1 \mathrm{~g} / \mathrm{L}$ fructose and $18.9 \mathrm{~g} / \mathrm{L}$ glucose) for 3 months prior to implantation. For clinical relevance CXCR2smi and anti-PD1 therapeutic intervention was started at 14 days post implantation when small macroscopic tumours are present. Mice were then harvested at 28 days post implantation or left to reach an approved humane endpoint. To deplete $\mathrm{CD} 8^{+}$cells, mice received either anti-CD8a (Biolegend, 53-6.7) or IgG control (Biolegend, RTK2758; twice weekly i.p. 200 $\mu$ g) for 7 days after the initial 7 days of treatment with CXCR2smi and anti-PD1. Neutralization of XCL1 to deplete CDC1 cells; $50 \mu \mathrm{g}$ of anti-XCL1 (R\&D systems MAB486) or isotype-matched control antibodies (R\&D systems MAB006) were injected i.p. twice a week for 2 weeks in combination with AZD5069/anti-PD1 therapy. Immature bone marrow neutrophil enrichment was performed with a single dose of lipopolysaccharide (LPS) from E. coli_0111:B4; (1 mg/kg, i.p.) or PBS control and femurs collected after

7344 hours. Polymorphonuclear fraction of bone marrow from PBS and LPS treated mice isolated by percol density centrifugation. High density fraction, containing predominantly neutrophils was washed, resuspended, counted and $1 \times 10^{7}$ cells i.v. injected twice weekly for 2 weeks into orthtopic NASH-HCC anti-PD1 treated mice. Tumour burden was calculated by measuring the size of the tumour in three perpendicular planes using digital callipers. 
740 For the DEN/ALIOS model, WT C57BL/6 mice, bred in house were injected with a single dose of DEN at $80 \mathrm{mg} / \mathrm{kg}$ by i.p. injection at 14 days of age. Mice were placed on either the ALIOS diet consisting of an irradiated high trans-fat diet composed of $22 \%$ hydrogenated vegetable (Envigo, TD.110201) and sugar water or a control diet at 60 days of age. For clinical relevance, treatment with AZD5069 (AstraZeneca; 250 mg/mL in 0.5\% Hydroxypropyl Methylcellulose (HPMC), 0.1\% Tween 80; 100 mg/kg, o.g.) twice daily, or vehicle (0.5\% HPMC, $0.1 \%$ Tween 80 ; o.g.) twice daily, anti-PD1 (Biolegend, RMP114; $200 \mu$ g, i.p.) bi-weekly or IgG (Biolegend, RTK2758; $200 \mu$ g, i.p.) bi-weekly was used to treat the mice. To deplete CD8 ${ }^{+}$cells in AZD5069/anti-PD1 DEN/ALIOS mice, mice received either anti-CD8a (Biolegend, 53-6.7) or IgG control (Biolegend, RTK2758; first dose i.p. 400 $\mu$ g and then twice weekly i.p. $200 \mu$ g) from day 242 until harvest at day 284.

750

For the long-time CD-HFD feeding model, 5-week-old C57BL/6 mice (male) were fed choline-deficient high-fat diet (CD-HFD) (Research Diets; D05010402) for 13 months to induce NASH-HCC. For therapeutic intervention, anti-PD1 (BioXcell, RMP1-14; $200 \mu$ g, i.p.) bi-weekly, IgG (BioXcell, LTF-2; 200 $\mu$ g, i.p.) bi-weekly, or vehicle (PBS; i.p.) bi-weekly, was used to treat the mice for 8 weeks.

For acute-DEN experiments, 12-15 week old WT C57B6/J mice bred in house or ordered in from Charles River were treated with a single dose of anti-PD1 or IgG control (dosing as above for DEN/ALIOS model) and/or AZD5069 or vehicle control (dosing as above for DEN/ALIOS model) twice daily. The following day mice received a single high-dose DEN injection $(100 \mathrm{mg} / \mathrm{kg}$, i.p.) and tissues were harvested the following morning.

For PBS-control and LPS-induced acute-inflammatory models, data is described in full in Mackey, et al., 2021. Briefly, 8-10 week old WT C57BL/6 mice ordered in from Charles River were injected with a single dose of PBS (i.p) or LPS (E. coli, 0111:B4; 1 mg/kg, i.p.) and tissues were harvested after 24 hours.

\section{Human sample ethical approval}

767 Collection and use of human tissue were ethically approved by the North East - Newcastle and North

768 Tyneside 1 Research Ethics Committee. Human liver tissue from surgical resections was obtained 769 under full ethical approval (H10/H0906/41) and through the CEPA biobank (17/NE/0070) and used 770 subject to patients written consent. HCC tumour and non-tumour biopsy tissue was obtained under 771 full ethical approval as approved by the Newcastle and North Tyneside Regional Ethics Committee, the Newcastle Academic Health Partners Bioresource (NAHPB) and the Newcastle upon Tyne NHS 
773 Foundation Trust Research and Development (R\&D) department. (Reference numbers: 10/H0906/41; NAHPB Project 48; REC 12/NE/0395; R\&D 6579; Human Tissue Act licence 12534).

\section{Scoring of tumour burden}

777

For the DEN/ALIOS model, whole livers were weighed, then dissected into 3-4 sections and liver tumours were scored using digital callipers. For the orthotopic NASH-HCC model, whole livers were weighed and tumours were scored with digital callipers in three dimensions for calculating tumour volume $\left(\mathrm{mm}^{3}\right)$.

781

\section{Sample processing and staining for flow cytometry and FACS}

Tissues were collected in ice-cold PBS. Blood samples were collected into EDTA coated syringes and immediately treated with Erythrocyte Lysis Buffer containing $\mathrm{NH}_{4} \mathrm{Cl}, \mathrm{KHCO}_{3}$ and $\mathrm{Na}_{2} \mathrm{EDTA}$ (SigmaAldrich) in $\mathrm{dH}_{2} \mathrm{O}, \mathrm{pH}$ 7.2-7.4. Non-tumour liver and tumours were manually diced. Tumours were digested using a mouse tumour dissociation kit in GentleMACS C digestion tubes with a GentleMACS tissue dissociator (Miltenyi Biotec). Enzyme activity was neutralised by addition of cold RPMI/2\% FBS and suspension was dispersed through a $70 \mu \mathrm{m}$ cell strainer. Single cell suspensions were treated with RBC lysis buffer. Cells were blocked with CD16/32 (BioLegend) as required and stained with directly conjugated antibodies (listed below) for 25 minutes at $4{ }^{\circ} \mathrm{C}$ in the dark in PBS/1\% BSA/0.05\% $\mathrm{NaN}_{3}$. Zombie NIR (zNIR) fixable viability (1:1000; BioLegend) was added to exclude dead cells. For surface antigen staining only, cells were fixed in $2 \%$ paraformaldehyde. For intracellular staining, cells were fixed and permeabilized using the FOXP3/Transcription Factor staining buffer set (ThermoFisher), then staining of intracellular proteins. For cell counts, 10,000 AccuCount fluorescent particles (Spherotech) were added to each sample. All experiments were performed using a BD LSRFortessa flow cytometer using BD FACSDive ${ }^{\mathrm{TM}}$ Diva software. Data were analysed using FlowJo software version 10.7.1.

797

All antibodies were purchased from BioLegend, except for CD101-PE, CD101-PE/Cy7, CD45-SB600 and IFNY-PE/Cy7, which were obtained from eBioscience and Siglec-F-APC/Cy7, Siglec-F-Bv605, Ly6GBuv395, CD11b-FITC, and CD162-Bv510, which were obtained from BD Biosciences.

801 General immune panel I: CXCR2-FITC (1:100, SA04E51), CD45-Bv711 (1:200, 30-F11), Siglec-F-Bv605 802 (1:200, E50-2440), CD31-PerCPCy5.5 (1:100, 390), CD3e-Bv421 (1:100, 145-2C11), Ly6G-Buv395 803 (1:100, 1A8), CD8 $\alpha-A F 700$ (1:100, 53-6.7), CD19-AF647 (1:100, 6D5), CD4-PE/Cy7 (1:200, RM4-5), 804 Nkp46-PE (1:100, 29A1.4), fixable viability dye zNIR. 
806 General immune panel II: CXCR2-FITC (1:100, SA04E51), XCR1-Bv785 (1:100, ZET), Ly6C-Bv711 (1:200, 807 HK1.4), CD11b-Bv650 (1:400, M1/70), CD11c-Bv605 (1:200, N418), F4/80-Bv510 (1:200, BM8), CD115808 Bv421 (1:200, AFS98), CD45-AF700 (1:200, 30-F11), CD172a-AF647 (1:100, P84), CD64-PE/Cy7 (1:200, 809 X54-5/7.1), IA/IE-Bv421 (1:200, M5/114.15.2), CD26-PE (1:200, H194-112), fixable viability dye zNIR. 810

811 Tail bleed panel: Siglec-F-APC/Cy7 (1:200, E50-2440), CD45-Bv711 (1:200, 30-F11), CD62L-Buv395 812 (1:200, MEL-14), CD101-PE/Cy7 (1:200, Moushi101), Ly6G-AF647 (1:400, 1A8), CD11b-Bv650 (1:400, 813 M1/70), CXCR2-FITC (1:100, SA04E51), CD3e-Bv421 (1:100, 145-2C11), CD4-Bv605 (1:100, RM4-5), 814 CD8 $\alpha$-AF700 (1:100, 53-6.7), PD-1-Bv785 (1:100, 29F.1A12), CD44-PerCP/Cy5.5 (1:100, IM7), PD-L1-PE 815 (1:100, 10F.9G2), fixable viability dye zNIR.

816

817 Neutrophil panel: Siglec-F-APC/Cy7 (1:200, E50-2440), CD117-PE/Cy7 (1:100, 2B8), Ly6G-AF647 818 (1:400, 1A8), CD11b-Bv650 (1:400, M1/70), CXCR2-FITC (1:100, SA04E51), CD101 (1:200, Moushi101), 819 CD45-Bv711 (1:200, 30-F11), PD-1-Bv785 (1:100, 29F.1A12), CD62L-Buv395 (1:200, MEL-14), fixable 820 viability dye zNIR.

821

822 T cell panel: CD44-PerCP/Cy5.5 (1:100, IM7), CD4-FITC (1:100, RM4-5), PD-1-Bv785 (1:100, 29F.1A12), 823 T-bet-Bv711 (1:200, 4B10), IL-17-Bv650 (1:400, TC11-18H10.1), CD45-SB600 (1:200, 30-F11), CD3ع824 Bv421 (1:100, 145-2C11), CD19-Buv805 (1:400, 6D5), CD62L-Buv395 (1:200, MEL-14), CD8 $\alpha-A F 700$ 825 (1:100, 53-6.7), GranzymeB-AF647 (1:50, GB11), IFNY-PE/Cy7 (1:200, XMG1.2), CD69-PE (1:100, 826 H1.2F3) fixable viability dye zNIR.

827

828 DC and macrophage panel: CD45-AF700 (1:200, 30-F11), CD11C-Bv605 (1:200, N418), CD26-PE (1:200, 829 H194-112), XCR1-Bv785 (1:100, ZET), CD103-PerCP/Cy5.5 (1:100, 2e7), IA/IE-Bv421 (1:200, 830 M5/114.15.2), CD86-FITC (1:200, GL-1), CD11b-Bv650 (1:400, M1/70), CD172a-AF647 (1:100, P84), 831 Ly6C-Bv711 (1:200, HK1.4), F4/80-Bv510 (1:200, BM8), CD64-PE/Cy7 (1:200, X54-5/7.1), fixable 832 viability dye zNIR.

833

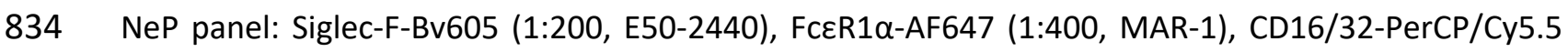
835 (1:100, 93), Ly6B-FITC (1:400, 74), CD11a-PE (1:400, M17/4), Ly6G-PE/Cy7 (1:400, 1A8), CD162-Bv510 836 (1:400, 2PH1), CD115-Bv421 (1:200, AFS98), fixable viability dye zNIR.

838 Fluorescent-Activated Cell Sorting (FACS) panel: CD45-SB600 (1:200, 30-F11), CD48-PE/Cy7 (1:200, 839 HM48-1), Ly6G-AF647 (1:400, 1A8), CD11b-FITC (1:400, M1/70), CD3e-PE (1:100, 145-2C11), DAPI. 
841 Neutrophil and T cell RNA isolation and sequencing and analysis

842 Ly6G $^{+}$neutrophils and $\mathrm{CD3}^{+} \mathrm{T}$ cells were FACS-sorted from the peripheral blood and tumours of 843 DEN/ALIOS mice. Purity of isolated populations was analysed by flow cytometry at $\geq 97 \%$. RNA was 844 isolated using the Rneasy Micro Kit (Qiagen) according to the manufacturer's protocol. RNA quality 845 and quantity was checked on an Agilent Bioanalyser 2100 using RNA Pico 6000 chip. Libraries for 846 cluster generation and DNA sequencing were prepared following the TaKaRa SMARTer Stranded Total 847 RNA-Seq Kit- Pico Input Mammalian v2 protocol. Quality and quantity of the DNA libraries was 848 assessed on an Agilent 2200 Tapestation (D1000 screentape) and Qubit (Thermo Fisher Scientific) 849 respectively. The libraries were run on the Illumina Next Seq 500 using the High Output v2.5, 75 cycles 850 kit ( 2 × 36cycles, paired-end reads). Illumina data were demultiplexed using bcl2fastq version 2.19.0, 851 then adaptor sequences were removed using Cutadapt version 0.6.4 and quality checked using fastqc 852 version 0.11.8. Next, paired end reads were aligned to the mouse genome version $\mathrm{GRCm} 38.95$ using 853 HISAT2 version 2.1.0, and gene expression was determined using Htseq version 0.11.2. Differential 854 expression analysis was performed using the R package DESeq2 version 1.22.2. Accurately identified 855 genes were classed as those with $\geq 2$ reads/million in $\geq$ half of the biological replicas in at least one 856 experimental condition. DEGs were identified as those with a $p$-value $\leq 0.05$ and fold change $\geq 1.5$ 857 between compared data sets. Gene ontology and pathway analysis was performed using MetaCore 858 (Clarivate Analytics).

859

\section{Laser capture micro-dissection RNA-Sequencing}

861 Formalin-fixed, paraffin-embedded (FFPE) 10 $\mu \mathrm{m}$ liver sections on Zeiss membrane slides were 862 dewaxed, rehydrated through graded alcohols, and then stained with haematoxylin and eosin (H\&E). 863 Tumour tissue was excised using Zeiss PALM microbeam laser capture microdissection microscope. 864 RNA was then isolated using the High Pure FFPE RNA Micro Kit (Roche). RNA quality and quantity was 865 checked using the DV200 metric (REF) on an Agilent Bioanalyser 2100 using RNA Pico 6000 chip. Total 866 RNA sequencing libraries were prepared using the SMART-Seq Stranded kit [Takara Bio] following the 867 manufacturer's protocol. Libraries were quantified using and a Tapestation 4200 [Agilent] and Qubit 8684 [Life Technologies] and equimolar pooled and sequenced at $>30$ million $100 \mathrm{bp}$ single reads per 869 sample on a NovaSeq 6000 using an 100 cycle SP flow cell [Illumina]. Data for individual samples was 870 demultiplexed into separate FASTQ files using Illumina's bcl2fastq software. Data were analysed as 871 above (Neutrophil RNA isolation and sequencing and analysis).

872 


\section{Whole tumour RNA-Sequencing and Analysis}

875 Whole tumor and healthy tissue was snap frozen and stored at $-80 \mathrm{C}$. Tissue was homogenized using 876 the Precellys Evolution homogenizer and bulk RNA was isolated using the Rneasy Kit (Qiagen) 877 according to the manufacturer's instructions, including the optional Dnase I step. RNA quality and 878 quantity was analysed on a Nanodrop 2000 (Thermo Fisher Scientific) and an Agilent 2200 TapeStation 879 (D1000 screentape). Only samples with a RIN value $>7$ were used for library preparation. Libraries 880 were prepared using the TruSeq stranded mRNA Kit. Library quality and quantity were assessed using 8812200 TapeStation (Agilent) and Qubit (ThermoFisher Scientific). The libraries were then run on an 882 Illumina NextSeq 500 using the High Output 75 cycles kit (2x36cycle paired end reads). Data were analysed as above (Neutrophil RNA isolation and sequencing and analysis).

884

RNA isolation, cDNA synthesis and qRT-PCR

$886 \mathrm{Ly}_{6 \mathrm{G}}^{+}$neutrophils sorted from orthtopic NASH-HCC tumours were snap frozen and stored at $-80^{\circ} \mathrm{C}$. 887 RNA was isolated using the Rneasy Kit (Qiagen) according to the manufacturer's instructions, including the optional Dnase I step. cDNA synthesis was performed using the iScript cDNA synthesis kit (BioRad) according to the manufacturer's instructions. Real time PCR was performed using SYBR Green jumpstart ready mix and the primers listed in (Supplementary Table 1).

891

Publicly available gene expression dataset analysis

893 Publicly available RNA-seq datasets of differentially expressed genes from RNA-seq performed on 894 biopsies from patients with NASH F0/F1 and NASH F4 were accessed. Gene ontology analysis was 895 performed using genes significantly upregulated in patients with advanced disease. NASH-related 896 HCCs, non-NASH-HCCs, as well as NASH liver human samples were previously described ${ }^{22,44}$. 897 Transcriptomic data from human NASH liver $(n=74)$ and NASH-HCC $(n=53)$ samples were used to assess 898 the expression of CXCR2 and key neutrophil-related cytokines in both tissues. The single sample Gene 899 Set Enrichment Analysis (sSGSEA) module of GenePattern was used to determine the enrichment 900 scores of immune-related gene signatures and signatures associated to response to immune 901 checkpoint inhibitors ${ }^{22,44}$. Differentially expressed genes from RNA-seq performed on peripheral blood 902 and liver Ly6G ${ }^{+}$neutrophils, and peripheral blood Ly6G ${ }^{\text {Int }}$ and Ly6G ${ }^{\mathrm{Hi}}$ neutrophils, from LPS-treated 903 acute systemic inflammatory response and PBS-control treated mice were accessed. Gene ontology 904 and pathway analysis was performed using MetaCore (Clarivate Analytics).

905 
DNA was isolated from Hep53.4 cells using the QIAamp DNA kit (Qiagen) as per manufacturers

908

909

910

911

912

913

914

915

916

917

918

919

920

921

922

923

924

925

926

927

928

929

930

931

932

933

934

935

936

\section{7}

938

939

940 instruction and then sent for exome sequencing (Novogene). Raw read outputs were then passed through FastQC and FastQ screen for quality control. Raw reads were then aligned to the mm10 genome assembly using the Burrow-Wheeler aligner (BWA-MEM) software. Duplicate reads were identified using Picard tools and base recalibration was performed using BaseRecalibrator. Variants were called using Mutect2, before analysis using Ensembl Variant Effect Predictor to identify the likely effects of genomic variants. Data were visualised using R version 4.0.2 using the maftools, tidyverse packages and MutationalPatterns packages. We extracted known drivers of HCC in human disease from the Catalogue of Somatic Mutations in Cancer (COSMIC) database. Using mouse:human homology mapping through ENSEMBL cross species annotations, we then identified mutations present in known cancer driver genes. To account for gene length, the number of mutations is presented as number of mutations per million base pairs.

\section{Histology and immunohistochemistry}

FFPE tissue sections were stained with H\&E using established protocols. Immunohistochemistry (IHC) was performed on FFPE sections that were dewaxed and rehydrated through graded alcohols. Endogenous peroxidases were then blocked using hydrogen peroxide/methanol solution. Antigen retrieval was performed using Tris-EDTA pH9. Endogenous avidin and biotin were blocked using the Avidin/Biotin blocking kit (Vector Laboratories, SP-2001) and non-specific binding was blocked using $20 \%$ swine serum in PBS. Antibodies were incubated at room temperature for 1 hour. Sections were washed and incubated in the appropriate biotinylated secondary antibody. Sections were then washed and incubated in Vectastain Elite ABC-HRP reagent (Vector Laboratories, PK-6100). Staining was visualised using DAB substrate kite (Vector Laboratories, SK-4100), counter stained with mayer haematoxylin and then mounted. CD3 (AbCam Ab16669, pH6 1:50), CD4 (eBioscience 14-9766-82, ER2 Leica, 1:500), CD8 (eBioscience 14-0808-82, ER2 Leica, 1:500), Ly6G (clone 1A8, 2B Scientific BE00751, ER2 Leica, 1:60000), LTF (Thermofisher, PA5-95513, 1:200), granzyme B (Abcam, ab255598, Clone: EPR22645-206, 1:100), CD8 (Abcam, ab217344, Clone: EPR21769, 1:100). Image analysis was performed using a Nikon Eclipse Upright microscope and NIS-Elements BR analysis software. A minimum of ten consecutive non-overlapping fields were imaged where possible.

\section{Automated IHC and analysis of human tissue}

Automated immunohistochemistry was performed using the Ventana Discovery XT platform. FFPE sections were dewaxed and rehydrated in EZ prep solution and then Tris-EDTA heat mediated antigen retrieval was performed. Endogenous peroxides and proteins were blocked using the Discovery 
941 Inhibitor CM. Sections were then incubated with primary antibodies (CD66b - Biolegend 305102,

942 CXCR2 - Sigma HPA031999) followed by the appropriate secondaries. Staining was visualised by 943 incubated slides in DAB followed by counterstaining with haematoxylin and then mounting. Sections 944 were scanned using a Leica Aperio scanner and the analysis performed using Aperio ImageScope slide 945 viewing software. Cell counts were performed on the whole tissue samples and normalised to the 946 total area analysed $\left(\mathrm{mm}^{2}\right)$.

947

\section{Imaging Mass Cytometry}

949 The following antibodies were used for imaging mass cytometry: CK18 (Thermofisher, PA5-14263), 950 F4/80 (Biorad, Cl:A3-1), CD3 (Biorad, CD3-12), Ki67 (Thermofisher, 14-5698-82), CD8 (Abcam, 951 EPR21769), CD45R (Thermofisher, 14-0452-82), MHCII (Thermofisher, 14-5321-81), aSMA 952 (ProteinTech, 14395-1-AP) and MPO (R\&D, AF3667). Ly6G was not used to identify neutrophils as it 953 was not compatible with IMC, instead MPO was used. Antibodies were first validated by 954 immunohistochemistry and immunofluorescence staining of FFPE tissue samples using a single 955 antigen retrieval method consisting of heat mediated antigen retrieval using the universal antigen 956 retrieval solution (Abcam). Based on the relative immunofluorescence signal antibodies were ranked and then pair with the appropriate metal for conjugation to maximise signal to noise ratio. Antibodies were conjugated to metals using MaxPar antibody conjugation kits (Fluidigm) following manufacturer's instructions. An antibody stabilisation solution was added before storage at $4^{\circ} \mathrm{C}$

960 (Candor Bioscience). The following metal-antibody conjugates were used: Nd145-CK18, Gd155-F4/80,

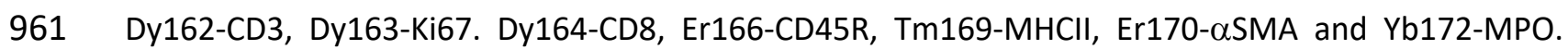

962 Conjugated antibodies were then validated signal by IMC in suspension mode (Helios) with antibody 963 capture beads (AbC Total compensation beads, Thermo Fisher). To confirm conjugation did not alter antibody binding efficiency, antibodies were validated once more by immunofluorescence.

966 Tumour tissue microarrays were dewaxed and rehydrated through clearene and graded alcohols.

967 Antigen retrieval was performed by immersing the slides in HIER Universal Antigen Retrieval reagent 968 (Abcam, ab208572) at $95^{\circ} \mathrm{C}$ for $20 \mathrm{~min}$. Slides were then washed and blocked with $3 \%$ BSA in $1 X$ PBS 969 for $45 \mathrm{~min}$. The cocktail of antibodies was prepared at the proper concentration of each one by diluting 970 it in $0.5 \%$ BSA in 1 X PBS and leaving it overnight at $4^{\circ} \mathrm{C}$ in a humidified tray. The slides were then washed 971 in $0.2 \%$ Triton X -100 in PBS with gentle agitation, followed by rounds of $1 X$ PBS. Nuclei were stained 972 using DNA intercalator (Ir191/193) at $0.313 \mu \mathrm{M}$ in PBS for 30min. Finally, the slides were washed in 973 ultra-pure water with gentle agitation and left air-drying at room temperature. 
975 The Hyperion Tissue Imaging module was aligned and coupled to the Helios mass cytometry 976 instrument and calibrated using the appropriate protocols (Fluidigm). Slide libraries were generated 977 using low resolution images to aid identification of regions of interest (ROIs). ROIs ( $1 \mathrm{~mm}^{2}$ in area) were 978 ablated from each tumour sample on the TMAs. Imaging data files were exported in MCD viewer 979 software (Fluidigm) as 16-bit single layer TIFFs. Single cell segmentation was performed using the 980 Bodenmiller pipeline, combining open source software llastik for machine learning-based pixel classification and Cell Profiler for actual single cell segmentation. Cell phenotype and interaction analysis was performed in HistoCat.

983

984

\section{Liver intravital microscopy}

985

Mice were anaesthetised and maintained using isoflurane in approximately $95 \%$ oxygen enriched air

986 generated using a medical oxygen scavenger (VetTech). Mice were placed on a heat mat at $37^{\circ} \mathrm{C}$ for the duration of the procedure. After loss of reflexes, the liver was exposed and a custom-built vacuum chamber fitted with a $13 \mathrm{~mm}$ glass cover slip placed onto the liver. Minimal suction (0.1-0.3 bar) was applied to stabilise the liver against the coverslip. Imaging was performed using an upright Zeiss LSM 880 Airyscan confocal microscope using a 20x/1 NA water immersion objective lens. Images were acquired in using a 32 channel Gallium arsenide phosphide spectral detector and signal was collected with a resolution of $8.9 \mathrm{~nm}$ over the visible spectrum. For visualisation of the vasculature and immune cell subtypes, fluorescently conjugated antibodies Ly6G (Biolegend, $5 \mu \mathrm{g}, 1 \mathrm{~A} 8$ ), CD45 (Biolegend, $5 \mu \mathrm{g}$,

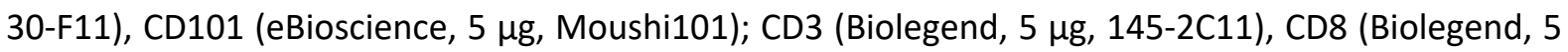
$\mu \mathrm{g}, 53-6.7)$, CD31 (Biolegend, $10 \mu \mathrm{g}, 390$ ), were injected i.v. through the tail vein prior to anaesthesia. Livers were imaged for up to 60 minutes with a z-stack of $12 \mu \mathrm{m}$. At the end of the imaging session, mice were humanely killed by cervical dislocation under anaesthesia. Spectral images were unmixed with Zen software (Carl Zeiss) using references spectra acquired from unstained tissue (tissue autofluorescence) or slides labelled with individual fluorescently conjugated antibodies. Time-lapse images were visualized and analysed using IMARIS software (Bitplane, Oxford Instruments, Abingdon UK; v9). For interaction analysis, spots were initially automatically created for Ly6G ${ }^{+}$cells and then corrected manually. Surfaces were initially automatically created for $\mathrm{CD}^{+}$cells and then corrected manually. $\mathrm{Ly}_{6 \mathrm{G}}+$ spots within $10 \mu \mathrm{m}$, measured from the cell centre, of a $\mathrm{CD}^{+}$surface were considered interacting.

\section{Live precision cut tumour-containing liver slices (PCLS) imaging}


1009 fluorescent antibodies CD31 (Biolegend, 1:100, 390), CD8 (Biolegend, 1:100, 53-6.7), Ly6G (Novus bio, 1010 1:100, 1A8), CD11c (Biolegend, 1:100, N418) and CD45 (Biolegend, 1:100, 30-F11) in complete medium 1011 (phenol-red free DMEM substituted with $1 \% \mathrm{FBS}$ ) for 20 minutes at $37^{\circ} \mathrm{C}$. Slices were imaged on a Zeiss 1012 LSM880 confocal microscope in a full incubation chamber at $37^{\circ} \mathrm{C}$ with $5 \% \mathrm{CO}$. Liver slices were 1013 imaged for 15-40min with z-stacks of $30 \mu \mathrm{m}$. Acquisition was performed with a 32 channel Gallium 1014 arsenide phosphide(GaAsP) spectral detector using 20x objective. Samples were excited 1015 simultaneously with 405, 488, 561 and 633nm wavelength laser lines and signal was collected onto a 1016 linear array of the $32 \mathrm{GaAsp}$ detectors in lambda mode with a resolution of $8.9 \mathrm{~nm}$ over the visible 1017 spectrum. Spectral images were then unmixed with Zen software (Carl Zeiss) using reference spectra 1018 acquired from unstained tissues (tissue autofluorescence) or beads labelled with single fluorophores.

\section{D images analysis}

1021 Timelapse images analysis and visualization was performed using Imaris (Bitplane). Neutrophils and T cells were detected and tracked using the 'spot detection' tool using either ${\text { Ly } 6 \mathrm{G}^{+}}^{+}$fluorescence intensities (neutrophils) or CD8 (CD8 ${ }^{+} \mathrm{T}$ cells). $\mathrm{CD} 11 \mathrm{c}^{+}$areas were segmented using the 'surface' tool to measure total $\mathrm{CD} 11 \mathrm{c}^{+}$volume. All spots and surfaces were checked manually to avoid any false detections. Cell behavior was determined using the track speed (indicating cell mobility). Interacting cells were determined as cells located $<15 \mu \mathrm{m}$ to other spots or surfaces.

\section{Data Availability}

1029

All data will be deposited with accession codes, unique identifiers or web links for publicly available datasets provided before publication.

1031

\section{Quantification and statistical analysis}

1033 Statistical analyses were performed using GraphPad Prism software (v9 GraphPad Software, La Jolla, 1034 CA, USA) and $R$ (version 3.5.1) performing tests as indicated and were considered statistically significant. P-values are included in figures.

\section{Acknowledgements:}

1039 General: We would like to that the core services and advanced technologies at the CRUK Beatson 1040 Institute with particular thanks to the Biological Services Unit, Molecular Services, Bioinformatics, 1041 Histology Department and the Beatson Advanced Imaging Resource. We would like to thank Danijela 1042 Heide, Jenny Hetzer for technical support for the CD-HFD NASH-HCC model. We would like to thank 1043 Catherine Winchester and Nathalie Sphyris for critical reading of the manuscript. We would like to 
1044

1045

1046

1047

1048

1049

1050

1051

1052

1053

1054

1055

1056

1057

1058

1059

1060

1061

1062

1063

1064

1065

1066

1067

1068

1069

1070

1071

1072

1073

1074

1075

1076

1077

thank the Newcastle University Comparative Biology Centre, Newcastle University Bioimaging Unit and the Newcastle University Flow cytometry core facility for technical assistance.

Funding: D.A.M., O.J.S., H.R. and T.G.B. were supported by program grant funding from CRUK (C9380/A26813). D.A.M. was supported by MRC program Grants MR/K0019494/1 and MR/R023026/1. D.A.M., O.J.S., H.R., T.G.B, J.B.G.M and J.L. are supported by a CRUK program grant (C18342/A23390). O.J.S. is funded by a CRUK grant (CRUK A21339). L.M.C. is funded by a CRUK grant (CRUK A23983). T.G.B. receives research funding support from AstraZeneca. T.G.B. and M.M. were funded by the Wellcome Trust (WT107492Z). H.R. was funded by CRUK Newcastle Experimental Cancer Medicine Center award (C9380/A18084). J.B.G.M., T.J. were supported by CRUK core funding (A17196 and A31287). J.L. was supported by funding from the Faculty of Medical Sciences, Newcatle University. D.G. is supported by the Newcastle CRUK Clinical Academic Training Programme. A.C. is funded by the W.E. Harker Foundation. R.E.F. is supported by a doctoral training grant from MICINN/MINECO (BES-2017-081286) and a mobility grant from Fundació Universitària Agustí Pedro i Pons. C.E.W. is supported by a Sara Borrell fellowship (CD19/00109) from the Instituto de Salud Carlos III (ISCIII) and the European Social Fund. P.K.H. is supported by a fellowship grant from the German Research Foundation (DFG, HA 8754/1-1). C.A.O. is supported by a predoctoral research scholarship from Fulbright España. J.M.L. is supported by grants from the NIH (RO1DK56621 and RO1DK128289), the Samuel Waxman Cancer Research Foundation, the Spanish National Health Institute (PID2019105378RB-100), through an Accelerator award in partnership between Cancer Research UK, Fondazione AIRC and Fundación Científica de la Asociación Española Contra el Cáncer (HUNTER, C9380/A26813), and by the Generalitat de Catalunya (AGAUR, SGR-1358).

Author Contributions: J.L., J.B.G.M., T.J. performed the majority of the laboratory-based work and analyses presented in the manuscript. E.R., T.M.D., F.F., W.C., K.G., A.H., C.N., S.L., M.L., R.P., P.K.H., R.E., C.E.W., M.R., C.F., D.F., S.R., N.W., M.M., A.C., D.G., A.F., D.M., A.F., X.C., N.M., C.A.F., X.L.R.I, A.J.M, M.M., R.R. performed a portion of the laboratory experiments and their related analyses. E.W.R., S.T.B., J.M.L., M.H., G.J.G. provided advice and/or contributed to the experimental design and writing. J.L., J.B.G.M., H.L.R., L.M.C., T.G.B., O.J.S., D.A.M. conceived the studies, designed the experiments and wrote the manuscript. All authors read and commented on the final manuscript. D.A.M., O.J.S., H.L.R., L.M.C., T.G.B. provided funding.

.



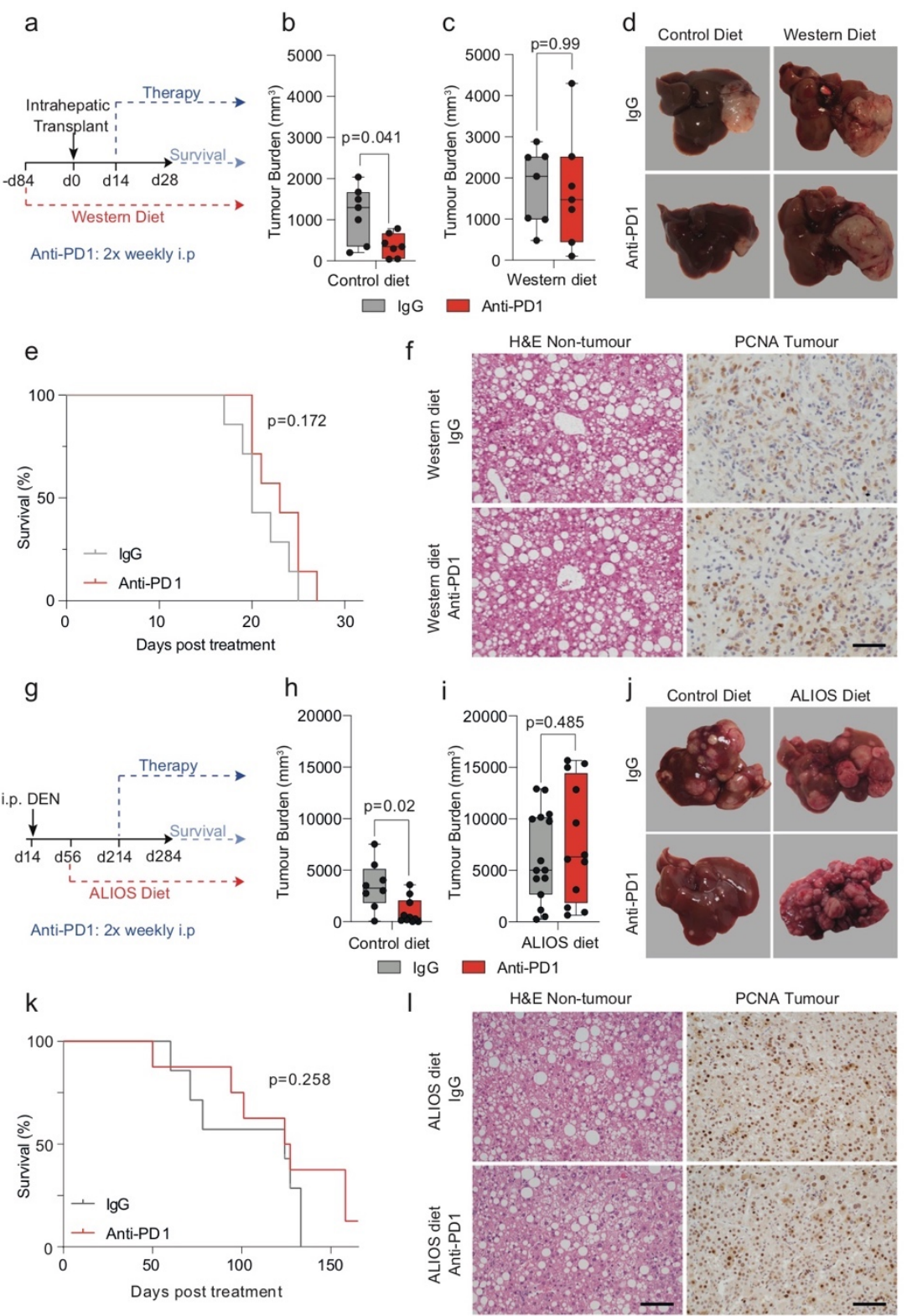

\section{Figure 1 - NASH-HCC is resistant to anti-PD1 immunotherapy}

a) Timeline schematic of the NASH-HCC model based on intra-hepatic injection of Hep53.4 HCC cells into C57BL/ 6 mice fed a control or Western diet. Cells were implaned 84 days after diet initiation, and anti-PD1 therapy was administered from day 14 post-transplantation.

b) Quantification of tumour burden $\left(\mathrm{mm}^{3}\right)$ at day 28 post-intrahepatic injection for orthotopic HCC mice fed a control diet and treated with IgG-control or anti-PD1. $n=7$ mice per condition. Mann-Whitney U-test.

c) Quantification of tumour burden $\left(\mathrm{mm}^{3}\right)$ at day 28 post-intrahepatic injection for orthotopic NASH-HCC mice fed a Western diet and treated with IgG-control or anti-PD1. $n=7$ mice per condition. Mann-Whitney $U$-test.

d) Representative images of livers at day 28 post-intrahepatic injection for orthotopic NASH-HCC mice fed a control or Western diet and treated with IgG-control or anti-PD1 from day 14. $n=7$ mice per condition.

e) Survival plot in orthotopic NASH-HCC mice fed a Western diet and treated with IgG-control or anti-PD1. $n=7$ mice per condition. Log-rank (Mantel-Cox) test. 
f) Representative images of H\&E-stained non-tumour livers and PCNA-stained tumours from orthotopic NASH-HCC mice fed a Western diet and treated with IgG-control or anti-PD1 at day 28 post-intrahepatic injection. $\mathrm{n}=7$ mice per condition. Scale bar $=100 \mu \mathrm{m}$.

g) Timeline schematic for the administration of Diethylnitrosamine (DEN) and the American lifestyle induced obesity syndrome (ALIOS) diet used to generate the autochthonous DEN/ALIOS NASH-HCC model with therapy timelines indicated.

h) Quantification of tumour burden $\left(\mathrm{mm}^{3}\right)$ at day 284 for DEN mice fed a control diet treated with IgG-control or anti-PD1. IgG $n=8$ mice. Anti-PD1 $n=10$ mice. Mann-Whitney U-test.

i) Quantification of tumour burden $\left(\mathrm{mm}^{3}\right)$ at day 284 for DEN mice fed the ALIOS diet treated with IgG-control or anti-PD1. IgG n=15 mice. Anti-PD1 n=12 mice. Mann-Whitney U-test.

j) Representative images of livers at day 284 for DEN mice fed either a control diet or the ALIOS diet treated with IgG-control or anti-PD1. IgG $n=15$ mice; Anti-PD1 $n=12$ mice.

k) Survival plot in DEN/ALIOS mice treated with IgG-control or anti-PD1 (censored at day 165 post-treatment). IgG $n=7$ mice. Anti-PD1 $n=8$ mice. Log-rank (Mantel-Cox) test.

I) Representative images of H\&E-stained non-tumour livers and PCNA-stained tumours from DEN/ALIOS mice treated with IgG-control or anti-PD1 at day 284. IgG $n=15$ mice; Anti-PD1 $\mathrm{n}=12$ mice. Scale bar $=100 \mu \mathrm{m}$.

Dots in Fig. 1b, c, h, i represent individual mice. 

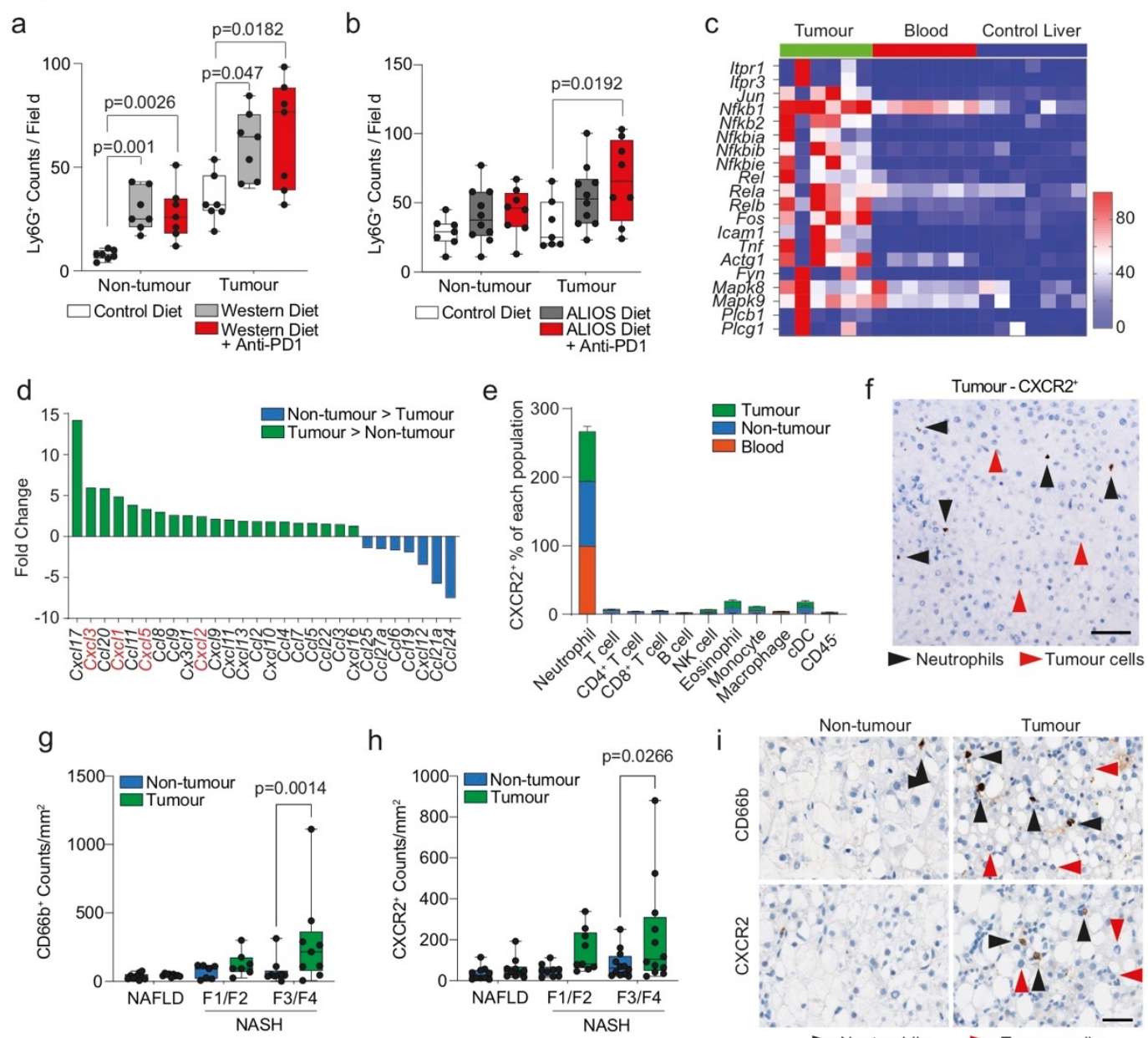

$\mathrm{h}$
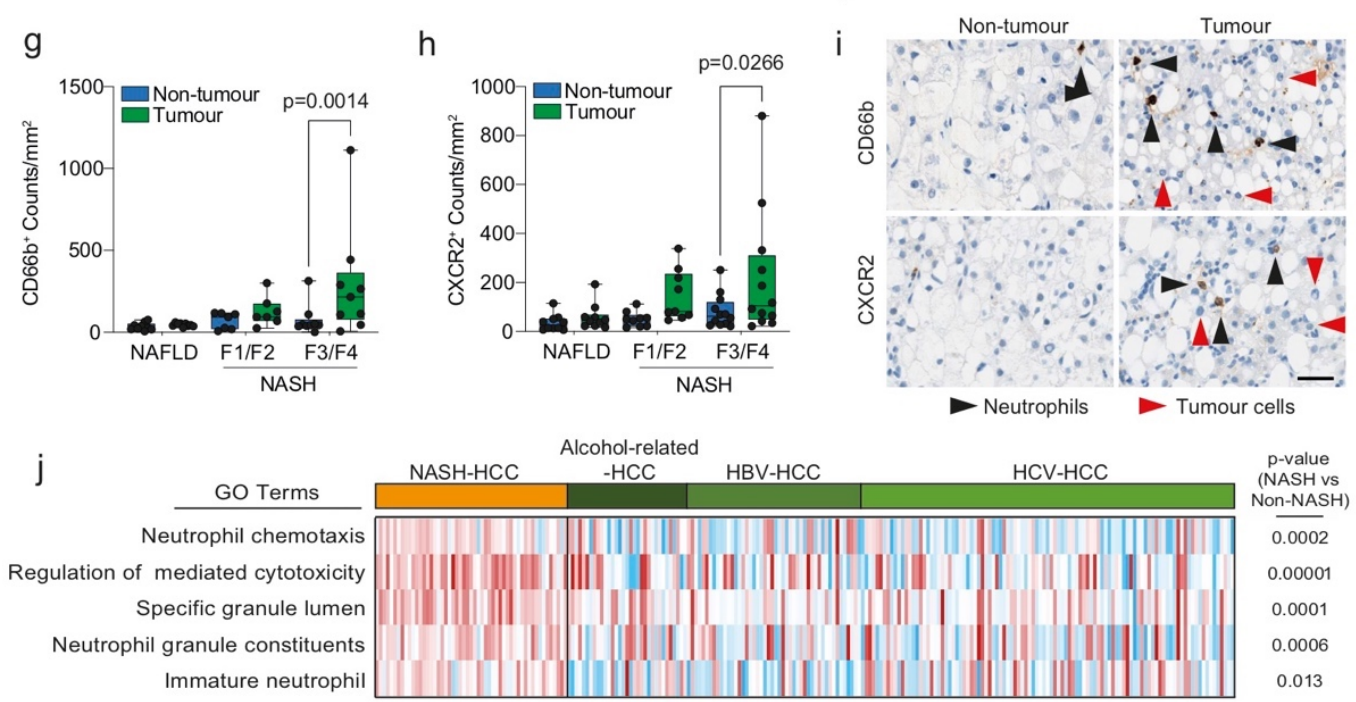

Low

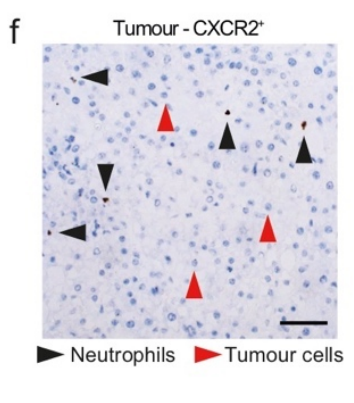

1115

1116

1117

1118

1119

1120

1121

1122

1123

1124

1125

1126

1127

1128

1129

1130

Figure 2 - NASH-HCC and anti-PD1 resistance is associated with CXCR2 $^{+}$neutrophils

a) Quantification of $\mathrm{Ly}_{6 \mathrm{G}}{ }^{+}$counts/field in non-tumour liver and tumours from IgG-control or antiPD1 treated orthotopic NASH-HCC mice. Control diet $n=7$, Western diet $n=7$, Western diet + Anti-PD1 $n=7$. Two-way ANOVA with Sidak's multiple comparisons test performed.

b) Quantification of $\mathrm{Ly}_{6 \mathrm{G}}{ }^{+}$counts/field in non-tumour liver and tumours from IgG-control or antiPD1 treated DEN/ALIOS mice. Control diet $n=7$, ALIOS diet $n=10$, ALIOS diet + Anti-PD1 $n=8$. Two-way ANOVA with Sidak's multiple comparisons test performed.

c) Heatmap showing row-scaled expression of DEGs associated with a pro-tumour neutrophil phenotype upregulated in DEN/ALIOS TANs compared with peripheral blood and control liver neutrophils. Data are from bulk Ly6G ${ }^{+}$neutrophil RNA-Seq.

d) Quantification of fold change for $C x \mathrm{Cl}$ and $\mathrm{Ccl}$ chemokine transcripts between DEN/ALIOS nontumour liver and tumour. Data are from bulk tissue RNA-Seq. Non-tumour $n=3$ mice; tumour $\mathrm{n}=5$ mice.

e) Flow cytometric quantification of $\mathrm{CXCR2}^{+}$as a percentage of cell populations in the peripheral blood, non-tumour liver and tumour in DEN/ALIOS mice. Peripheral blood $n=5$ mice; nontumour liver $n=5$ mice; tumour $n=3$ mice. Error bars represent Mean $\pm S E M$. 
f) Representative image of RNAscope in situ hydrisation staining of CXCR2 in DEN/ALIOS mouse tumours. Black arrowheads indicate positive infiltrating non-parenchymal cells and red arrows indicate negative tumour cells.

g) Quantification of $\mathrm{CD} 6 \mathrm{~b}^{+}$neutrophil counts $/ \mathrm{mm}^{2}$ in non-tumour liver and tumour by IHC of non-alcoholic fatty liver disease (NAFLD)-HCC and NASH-HCC patient resected tissue. Patients were graded as NAFLD F0 ( $n=9)$, NASH F1/F2 ( $n=7)$, and NASH F3/F4 ( $=9)$. Two-way ANOVA with Sidak's multiple comparisons test.

h) Quantification of $\mathrm{CXCR2}^{+}$counts $/ \mathrm{mm}^{2}$ in non-tumour liver and tumour by IHC from NAFLD$\mathrm{HCC}$ and NASH-HCC patient resected tissue. Patients graded as NAFLD F0 $n=10, N A S H$ F1/F2 $\mathrm{n}=9$ and NASH F3/F4 n=12. Two-Way ANOVA with Sidak's multiple comparisons test.

i) Representative IHC of $\mathrm{CD} 6 \mathrm{bb}^{+}$and $\mathrm{CXCR2}{ }^{+}$cells (indicatd by arrowheads) in non-tumour and tumour regions of NASH-HCC patient-resected-tissue. Scale bar $=100 \mu \mathrm{m} . \mathrm{n}=12$ patients.

j) Heatmap showing row-scaled expression of neutrophil-associated process networks for human NASH-HCC compared with HBV, HCV and alcohol-related HCC (non-NASH-HCC). Data from bulk tumour microarray. Total $n=237$ patients.

Dots in Fig. 2a, b, g, $\mathbf{h}$ represent individual mice. 
a

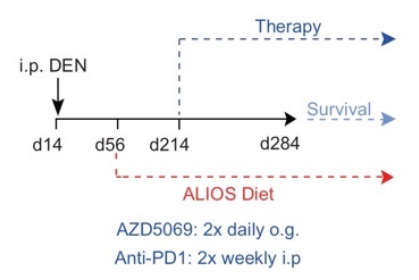

e

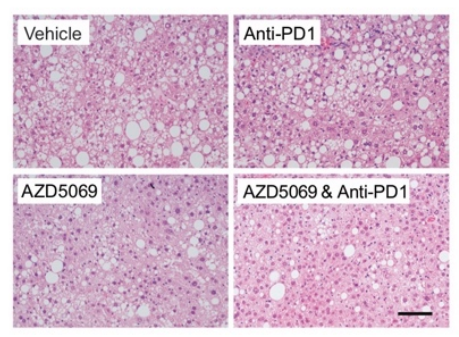

h

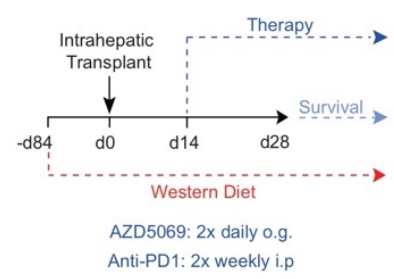

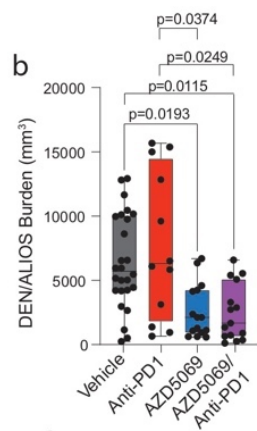

f
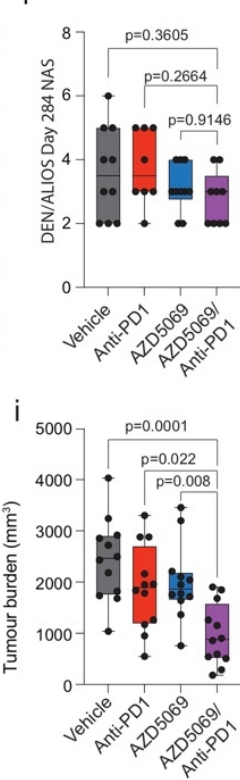
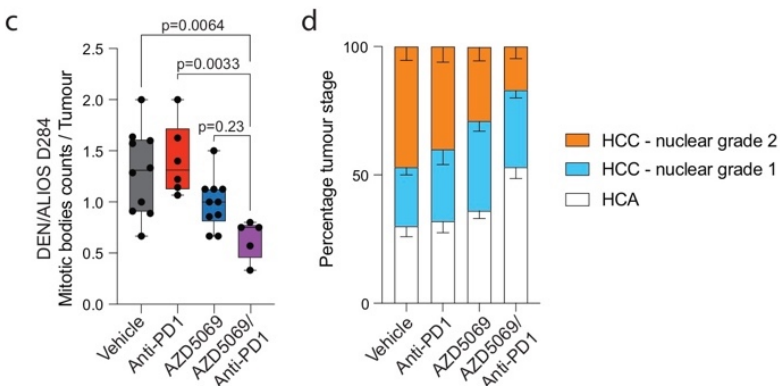

g
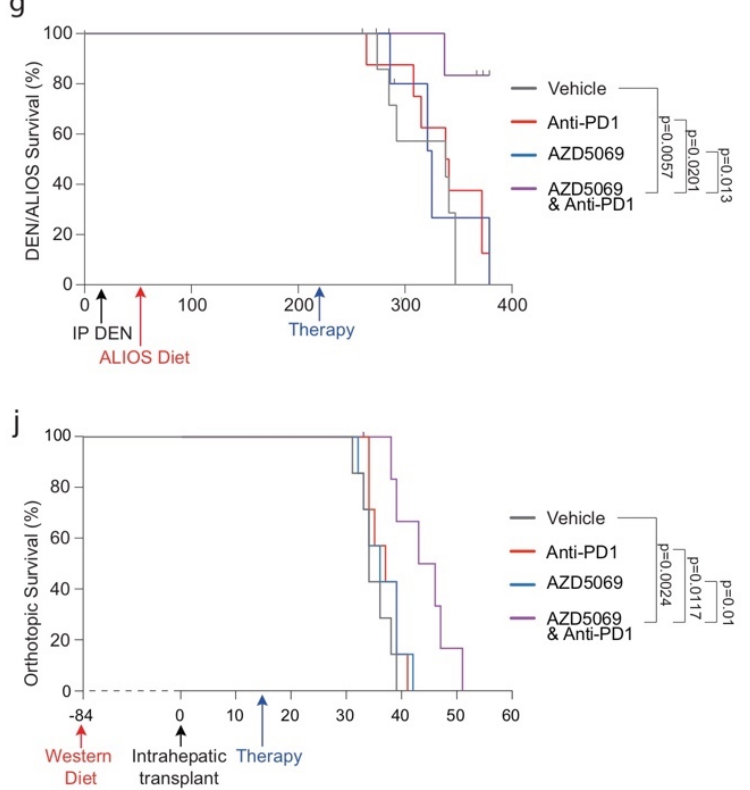

1155

1156

1157

1158

1159

1160

1161

1162

1163

1164

1165

1166

1167

1168

1169

1170

1171

1172

1173

1174

1175

1176

1177

1178

Figure 3 - Inhibition of CXCR2 ${ }^{+}$pro-tumour neutrophils re-sensitizes NASH-HCC to anti-PD1 therapy

a) Schematic for DEN/ALIOS model treatment regime.

b) Quantification of tumour burden $\left(\mathrm{mm}^{3}\right)$ for DEN/ALIOS mice at day 284 for each treatment arm. Vehicle $n=26$ mice; anti-PD1 $n=12$ mice; AZD5069 n=15 mice; AZD5069/anti-PD1 n=15 mice. Kruskal-Wallis test with Dunn's multiple comparisons test.

c) Quantification of average mitotic body counts per tumour for DEN/ALIOS mice at day 284. All tumours analysed. Vehicle $n=10$ mice; anti-PD1 $n=8$ mice; AZD5069 $n=10$ mice; AZD5069/antiPD1 $n=9$ mice. One-Way ANOVA with Tukey multiple comparisons test.

d) Quantification of tumour stage based on nuclear grading for DEN/ALIOS mice at day 284 for each treatment arm. All tumours analysed. Vehicle $n=10$ mice; anti-PD1 $n=8$ mice; AZD5069 $\mathrm{n}=10$ mice; AZD5069/anti-PD1 $\mathrm{n}=9$ mice. Mean \pm SEM.

e) Representative images of non-tumour liver H\&E for DEN/ALIOS mice. Vehicle $n=7$ mice; antiPD1 n=8 mice; AZD5069 n=7 mice; AZD5069/anti-PD1 n=9 mice. Scale bar $=100 \mu \mathrm{m}$.

f) Quantification of NAFLD activity score (NAS) in the livers for DEN/ALIOS mice at day 284. Vehicle $n=10$ mice; anti-PD1 n=8 mice; AZD5069 n=10 mice; AZD5069/anti-PD1 n=9 mice.

g) Survival plot for DEN/ALIOS mice (censored at day 365). Vehicle $n=7$ mice; anti-PD1 $n=8$ mice; AZD5069 n=7 mice; AZD5069/anti-PD1 n=9 mice. Log-rank (Mantel-Cox) test.

h) Schematic for orthotopic NASH-HCC model treatment regime.

i) Quantification of tumour burden $\left(\mathrm{mm}^{3}\right)$ for the orthotopic NASH-HCC mice at day 28. $\mathrm{n}=12$ mice per condition. One-Way ANOVA with Tukey's multiple comparisons test.

j) Survival plot in orthotopic NASH-HCC mice. For each treatment group $n=7$ mice, 1 mouse censored due to non-liver related medical issue. Log-rank (Mantel-Cox) test.

Dots in Fig. 3b, c, f, i represent individual mice. 
a

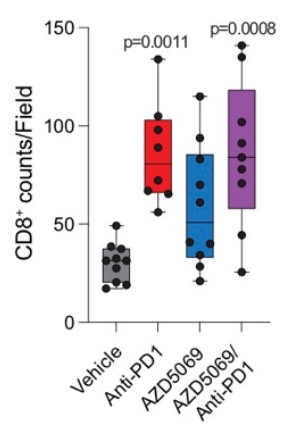

d

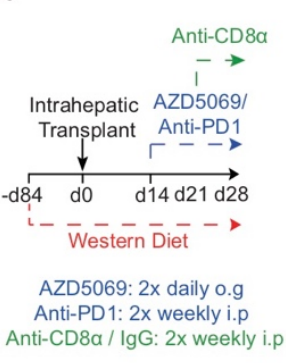

g

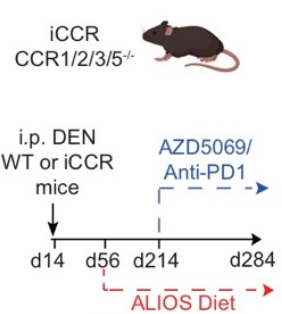

AZD5069: $2 x$ daily o.g Anti-PD1: $2 x$ weekly i.p

$\mathrm{k}$

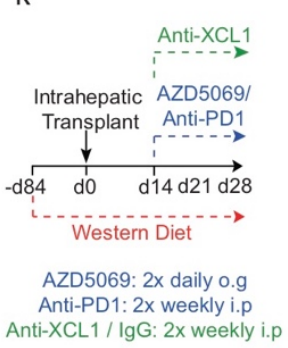

b

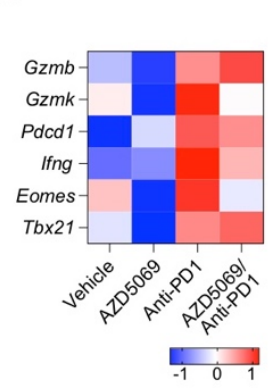

e

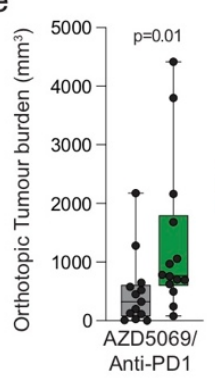

C
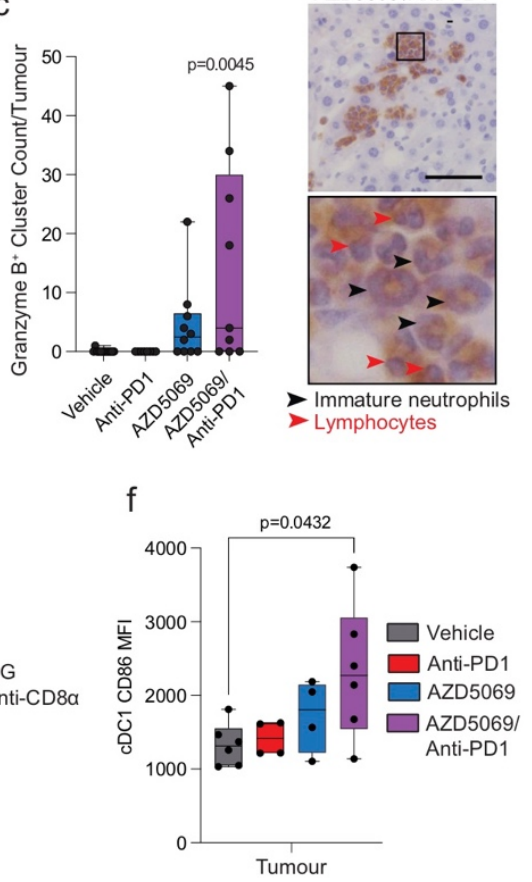

h i

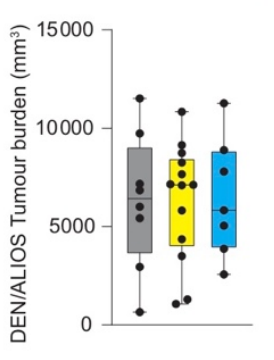

$\square$ WT - Vehicle

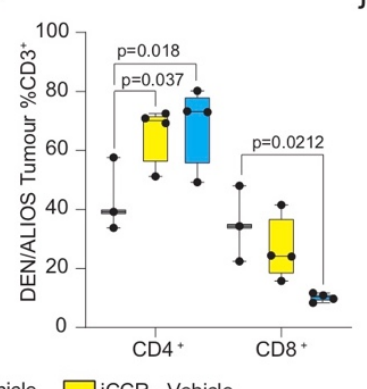

$\square$ iCCR - Vehicle

$\square$ iCCR - AZD5069/Anti-PD1
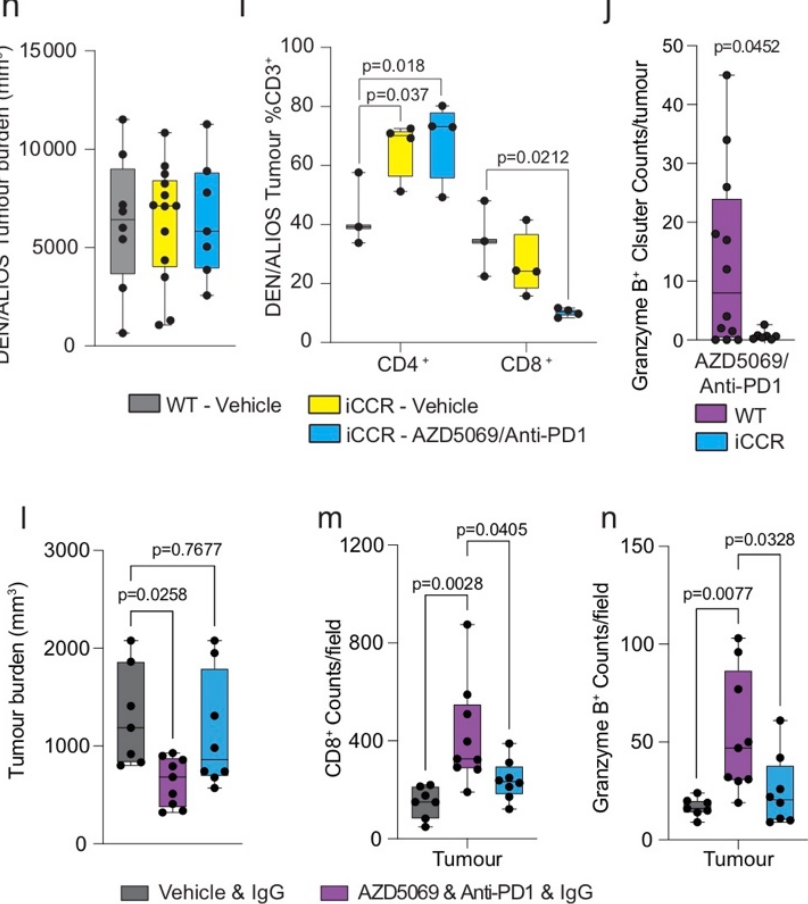

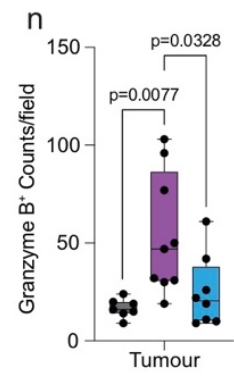

Tumour

Figure 4 - AZD5069/anti-PD1 therapy promotes an anti-tumour immune microenvironment.

a) Quantification of $C D 8^{+}$counts/field in tumours DEN/ALIOS model from each treatment arm. Vehicle $n=10$ mice; anti-PD1 $n=8$ mice; AZD5069 n=10 mice; AZD5069/anti-PD1 n=9 mice. One-Way ANOVA with Tukey multiple comparisons test.

b) Heatmap showing row-scaled expression of genes associated with $\mathrm{CD} 8^{+} \mathrm{T}$ cell activation and exhaustion for DEN/ALIOS treatment groups. Data are from bulk $\mathrm{CD}^{+}$Tumour associated T cells analysed by RNA-Seq.

c) Quantification of granzyme $\mathrm{B}^{+}$clusters by IHC in the tumours for DEN/ALIOS mice from each treatment arm at day 284 and representative images of granzyme $B^{+}$clusters in AZD5069/anti- 
PD1 treated mice (black arrow heads = banded neutrophils; blue arrow heads = lymphocytes). Vehicle $n=12$; anti-PD1 n=8; AZD5069 n=10; AZD5069/anti-PD1 n=9. One-Way ANOVA with Tukey's multiple comparisons test. Scale bar $=100 \mu \mathrm{m}$.

d) Timeline schematic for the anti-CD8a depletion regime in the orthotopic NASH-HCC model.

e) Quantification of tumour burden $\left(\mathrm{mm}^{3}\right)$ in orthotopic NASH-HCC mice treated with AZD5069/anti-PD1 and IgG-control or anti-CD8 $\alpha$ at day 28 post-intrahepatic injection. IgG $\mathrm{n}=13$. Anti-CD8 $\alpha \mathrm{n}=14$. Mann-Whitney U-test.

f) Flow cytometric quantification of CD86 median fluorescence intensity (MFI) of intratumoural $X C R 1^{+}$cDC1 cells from DEN/ALIOS mice treatment arms at day 284 . Vehicle $n=6$ mice; antiPD1 n=4 mice; AZD5069 n=4 mice; AZD5069/anti-PD1 n=6 mice. One-way ANOVA with Tukey multiple comparisons test.

g) Timeline schematic for the DEN/ALIOS regimen and targeted therapies in mice with compound deletion of Ccr1, 2, 3, 5 knockout mice, designated iCCR.

h) Quantification of tumour burden for DEN/ALIOS mice Vehicle-treated WT and iCCR, and AZD5069/anti-PD1 treated iCCR mice at day 284. WT-Vehicle $n=8$ mice; iCCR-Vehicle $n=13$ mice; iCCR-AZD5069/Anti-PD1 $n=7$ mice.

i) Flow cytometric quantification of $\mathrm{CD}^{+}$and $\mathrm{CD} 8^{+}$cells as a percentage of $\mathrm{CD}^{+}{ }^{+}$cells in tumours from WT-Vehicle, iCCR-Vehicle and iCCR-AZD5069/anti-PD1 treated DEN/ALIOS mice at day 284. WT-Vehicle $n=3$ mice; iCCR-Vehicle $n=4$ mice; iCCR-AZD5069/anti-PD1 $n=4$ mice. Two-way ANOVA with Tukey's multiple comparisons test.

j) Quantification of granzyme $B^{+}$clusters by IHC in WT and iCCR DEN/ALIOS mice treated with AZD5069/Anti-PD1 at day 284. WT $n=12$ mice. iCCR $n=7$ mice. Unpaired T-test.

k) Timeline schematic for the anti-XCL1 neutralisation regime in the orthotopic NASH-HCC model.

a) Quantification of tumour burden $\left(\mathrm{mm}^{3}\right)$ in orthotopic NASH-HCC mice treated with vehicle control and IgG-control or AZD5069/anti-PD1 and either IgG-control or anti-XCL1 at day 28 post-intrahepatic injection. Vehicle and IgG $n=7$. AZD5069/anti-PD1 and IgG-control $n=9$. AZD5069/anti-PD1 and anti-XCL1 n=8. One-Way ANOVA with Tukey's multiple comparisons test.

I) Quantification of $\mathrm{CD}^{+}$counts/field in tumours of orthotopic NASH-HCC mice treated with vehicle control and IgG-control or AZD5069/anti-PD1 and either IgG-control or anti-XCL1 at day 28 post-intrahepatic injection. Vehicle and IgG $n=7$. AZD5069/anti-PD1 and IgG-control $\mathrm{n}=9$. AZD5069/anti-PD1 and anti-XCL1 n=8. One-Way ANOVA with Tukey's multiple comparisons test.

m) Quantification of granzyme $B^{+}$counts/field in tumours of orthotopic NASH-HCC mice treated with vehicle control and IgG-control or AZD5069/anti-PD1 and either IgG-control or anti-XCL1 at day 28 post-intrahepatic injection. Vehicle and IgG $n=7$. AZD5069/anti-PD1 and IgG-control $\mathrm{n}=9$. AZD5069/anti-PD1 and anti-XCL1 $\mathrm{n}=8$. One-Way ANOVA with Tukey's multiple comparisons test. 

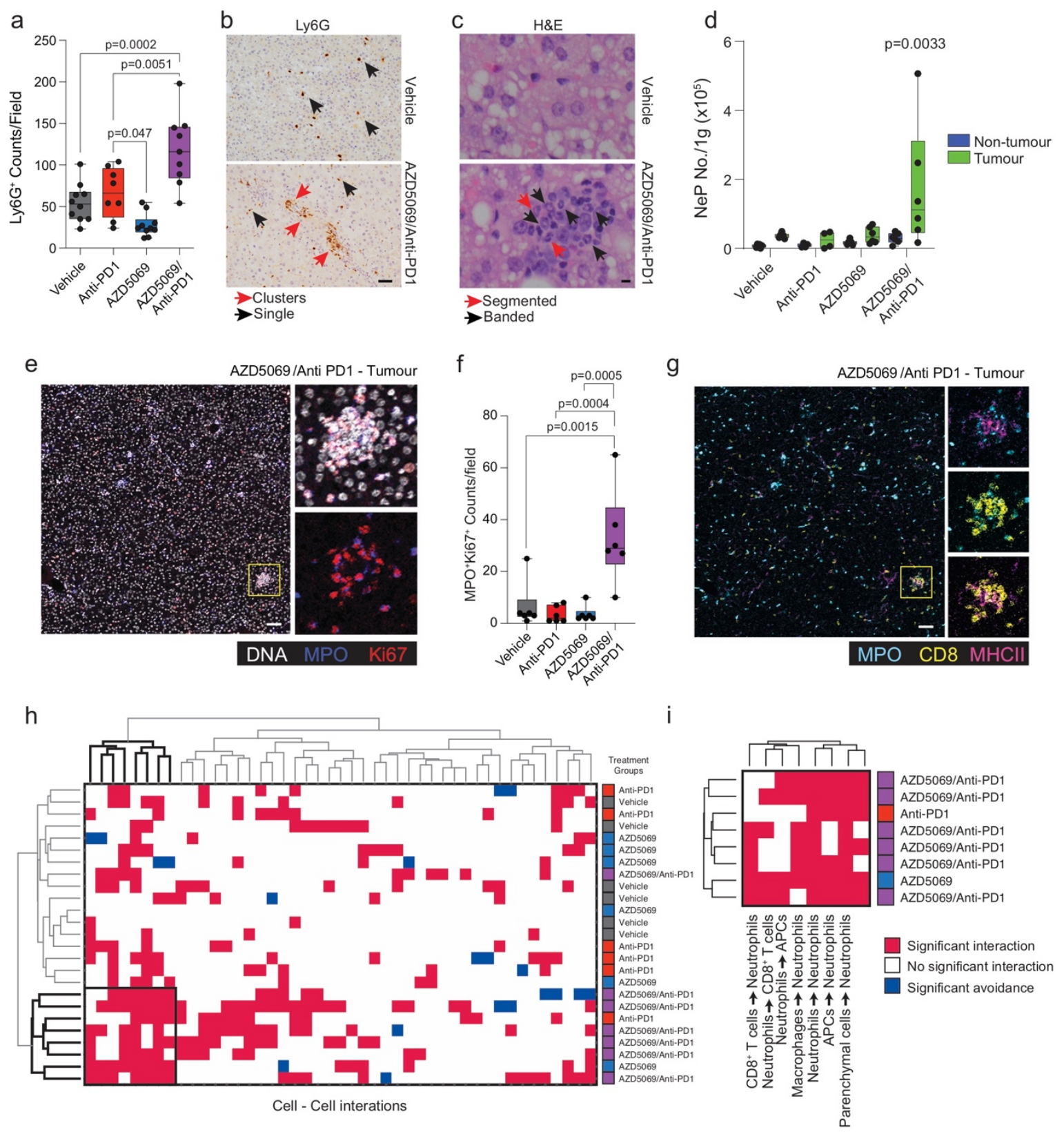

1232

1233

1234

1235

1236

1237

1238

1239

1240

1241

1242

1243

1244

1245

1246

1247

Figure 5 - AZD5069/anti-PD1 therapy promotes tumour neutrophil accumulation and the formation of intratumoural immunological hubs

b) Quantification of $\mathrm{Ly}_{6 \mathrm{G}}{ }^{+}$counts/field by IHC for DEN/ALIOS mice tumours at day 284 . Vehicle $\mathrm{n}=10$ mice; Anti-PD1 n=8 mice; AZD5069 n=10 mice; AZD5069/Anti-PD1 n=9 mice. One-Way ANOVA with Tukey's multiple comparisons test.

c) Representative images of $\mathrm{Ly}_{6 \mathrm{G}} \mathrm{G}^{+}$staining for DEN/ALIOS tumours. Vehicle $\mathrm{n}=10$ mice; AZD5069/Anti-PD1 $\mathrm{n}=9$ mice. Black arrows indicate single $\mathrm{Ly}_{6 \mathrm{G}}{ }^{+}$neutrophils; red arrows indicate clusters of $\mathrm{Ly} 6 \mathrm{G}^{+}$neutrophils. Scale bar $=100 \mu \mathrm{m}$.

d) Representative H\&E from Vehicle-control and AZD5069/anti-PD1 treated mouse tumours identifying clusters of neutrophils with banded (blue arrows) and segmented (black arrows) nuclear morphology. Vehicle $n=10$ mice; AZD5069/Anti-PD1 $n=9$ mice. Scale bar $=10 \mu \mathrm{m}$.

e) Flow cytometric quantification of NeP count/gram in non-tumour liver and tumour tissues for DEN/ALIOS mice for each treatment arm at day 284. Vehicle $n=6$ mice; anti-PD1 $n=4$ mice; AZD5069 n=6 mice; AZD5069/anti-PD1 n=6 mice. Two-way ANOVA with Sidak's multiple comparisons test. 
f) Representative intra-tumour IMC image for DEN/ALIOS mice treated with AZD5069/anti-PD1. DNA = white; $M P O=$ blue; Ki- $67=$ red. $n=6$ mice. Scale bar $=100 \mu \mathrm{m} . \mathrm{n}=6$ tumours.

g) Quantification of MPO $\mathrm{Ki}^{+}-67^{+}$counts/field for DEN/ALIOS tumours from IMC analysis. $\mathrm{n}=6$ mice per condition. One-Way ANOVA with Tukey's multiple comparisons test.

h) Representative intra-tumour IMC image for DEN/ALIOS mice treated with AZD5069/anti-PD1. $\mathrm{MPO}=$ cyan; $\mathrm{CD} 3=$ yellow; $\mathrm{MHCll}=$ purple. $\mathrm{n}=6$ mice. Scale bar $=100 \mu \mathrm{m}$.

i) HistoCAT neighbourhood clustering analysis performed using phonograph clustered cell populations across all four treatment arms where red indicated a significant interaction, blue indicates a significant avoidance and white indicated no significant interaction. Each column represents the interaction of two cell types. Each row represents an individual mouse. Vehicle $\mathrm{n}=6$ mice; anti-PD1 $\mathrm{n}=6$ mice; AZD5069 $\mathrm{n}=6$ mice; AZD5069/anti-PD1 $\mathrm{n}=7$ mice.

j) Magnified image of HistoCAT neighbourhood clustering analysis. Cluster showing specifically enriched cell-cell interactions. Cluster contains; anti-PD1 n=1 mouse; AZD5069 n=1 mouse; AZD5069/anti-PD1 $n=6$ mice. 7/8 cell-cell interactions characterised by antibodies used.

Dots in Fig. 5a, d, f represent individual mice. 

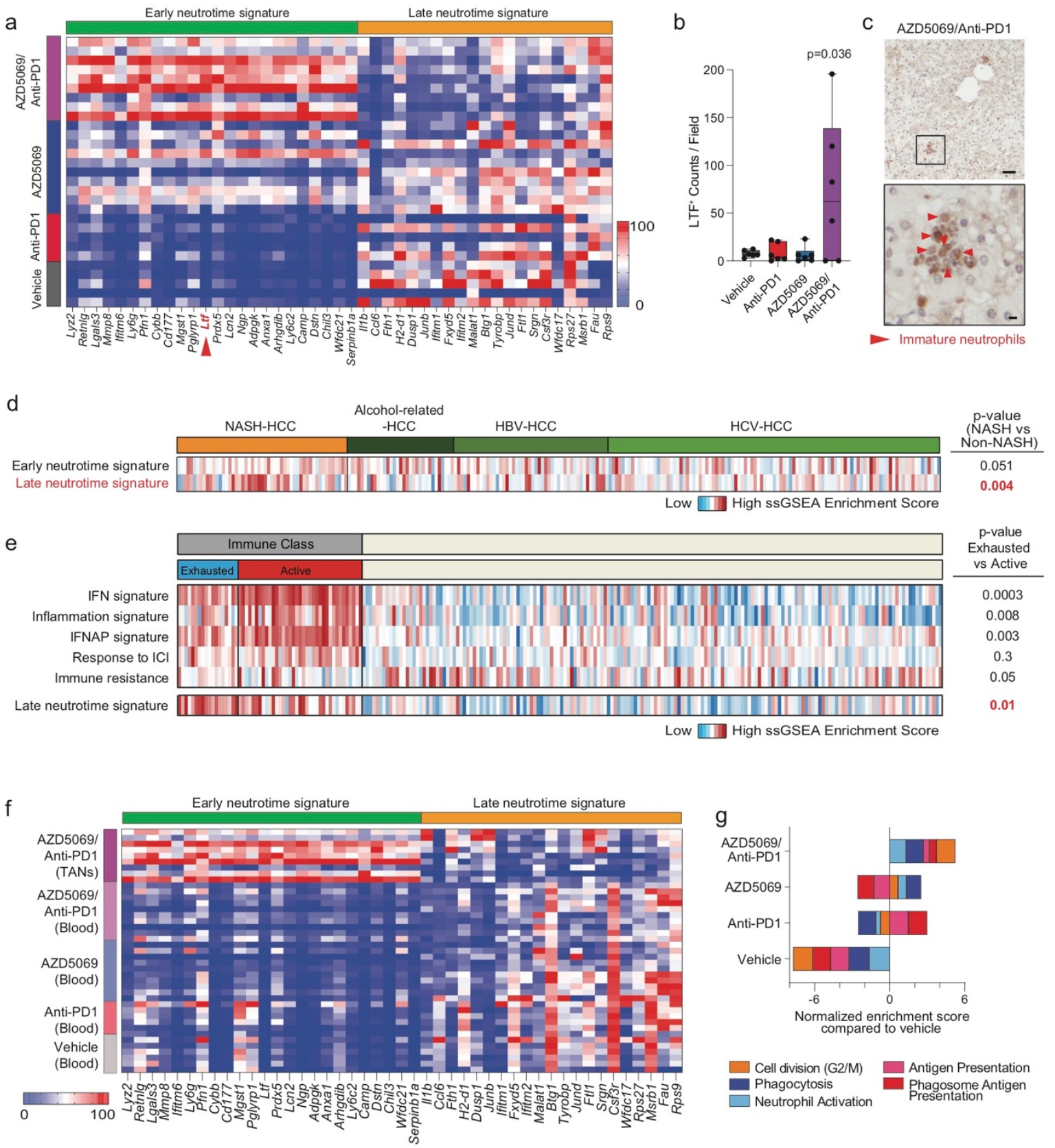

h
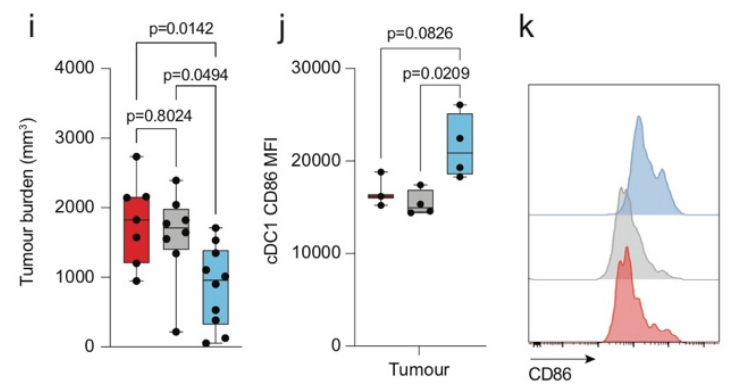

$\square$ Anti-PD1 $\square$ Anti-PD1 + mature neutrophils

Low High ssGSEA Enrichment Score

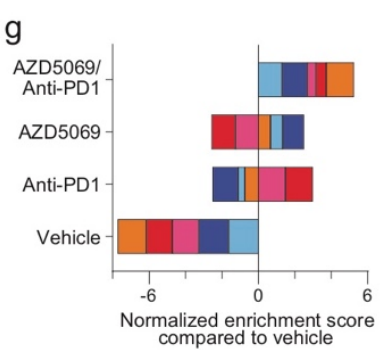

Cell division (G2/M)
Phagocytosis
Phagosome Antigen Neutrophil Activation
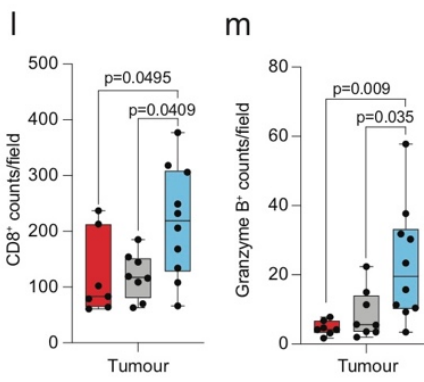

Anti-PD1 + immature neutrophils

Figure 6 - AZD5069/anti-PD1 combination therapy re-programmes the TAN phenotype.

a) Heatmap showing row-scaled expression of genes associated with late and early neutrotime for DEN/ALIOS mice TANs. Data are from bulk Ly6G ${ }^{+}$TANs analysed by RNA-Seq. 
b) Quantification of $\mathrm{LTF}^{+}$counts/field by IHC for DEN/ALIOS mice tumours at day 284. Vehicle $\mathrm{n}=6$ mice; Anti-PD1 $\mathrm{n}=6$ mice; AZD5069 $\mathrm{n}=6$ mice; AZD5069/Anti-PD1 $\mathrm{n}=6$ mice. One-Way ANOVA with Tukey's multiple comparisons test.

c) Represenative image of LTF positive neutrophils (red arrow) in the tumour of AZD5069/antiPD1 treated DEN/ALIOS mice at day 284. Scale bar top $=100 \mu \mathrm{m}$, bottom $=10 \mu \mathrm{m}$.

d) Heatmap showing row-scaled expression of early and late neutrotime signatures for human NASH-HCC compared with HBV, HCV and alcohol-related HCC (non-NASH-HCC). Data are from bulk tumour microarray. In total $n=237$ patients analysed.

e) Heatmap showing row-scaled expression of published HCC immune class signatures; IFN, inflammation, IFNAP, Response to $\mathrm{ICl}$ and immune resistance as well as the late neutrotime signature for human HCC active and exhausted immune subsets. Data are from bulk tumour microarray. In total $n=228$ patients analysed.

f) Heatmap showing row-scaled expression of genes associated with late and early neutrotime signatures (Grieshaber-Bouyer et al., 2021) for DEN/ALIOS peripheral blood neutrophils and AZD5069/Anti-PD1 treated TANs. Data are from bulk Ly6G ${ }^{+}$TANs analysed by RNA-Seq.

g) Gene set enrichment analysis (GSEA) showing normalised enrichment scores for TAN process networks highly enriched in; Anti-PD1 vs Vehicle (Phagosome Antigen Presentation and Antigen Presentation), AZD5069 vs Vehicle (Neutrophil Activation and Phagocytosis), and AZD5069/Anti-PD1 vs Vehicle (G2-M). Data are from bulk Ly6G ${ }^{+}$TANs analysed by RNA-Seq.

h) Timeline schematic for neutrophil based therapy treatment regime in the orthotopic NASHHCC model.

i) Quantification of tumour burden $\left(\mathrm{mm}^{3}\right)$ in orthotopic NASH-HCC mice treated with anti-PD1 and immature or mature neutrophils at day 28 post-intrahepatic injection. Anti-PD1 $n=7$. AntiPD1/Mature neutrophils $n=8$, anti-PD1/Immature neutrophils $n=10$. One-way ANOVA with Tukey's multiple comparisons test.

j) Flow cytometric quantification of CD86 median fluorscent intensity (MFI) of intraturmoural $X C R 1^{+}$CDC1 cells from orthotopic NASH-HCC neutrophil/anti-PD1 therapy mice at day 28 . Anti$P D 1 n=3$. Anti-PD1/Mature neutrophils $n=4$, anti-PD1/Immature neutrophils $n=4$. One-way ANOVA with Tukey's multiple comparisons test.

k) Representative histogram plot of CD86 median fluorscence intensity (MFI) of intraturmoural $X C R 1^{+}$CDC1 cells from orthotopic NASH-HCC neutrophil/anti-PD1 therapy mice at day 28 . AntiPD1 $n=3$. Anti-PD1/Mature neutrophils $n=4$, anti-PD1/Immature neutrophils $n=4$.

I) Quantification of intratumoural $\mathrm{CD}^{+}$counts/field in the tumours of orthotopic NASH-HCC neutrophil/anti-PD1 therapy mice at day 28. Anti-PD1 $n=7$. Anti-PD1/Mature neutrophils $n=8$, anti-PD1/Immature neutrophils $\mathrm{n}=10$. One-way ANOVA with Tukey's multiple comparisons test.

m) Quantification of Granzyme $B^{+}$counts/field in the tumours of orthotopic NASH-HCC neutrophil/anti-PD1 therapy mice at day 28. Anti-PD1 $n=7$. Anti-PD1/Mature neutrophils $n=8$, anti-PD1/Immature neutrophils $n=10$. One-way ANOVA with Tukey's multiple comparisons test.

Bulk DEN/ALIOS Ly6G ${ }^{+}$TAN RNA-Seq data in Fig. $6 \mathbf{b}$, e, $\mathbf{f}$ : Vehicle $n=6$ mice; anti-PD1 $n=5$ mice, AZD5069 n=10 mice; AZD5069 $n=8$ mice.

Dots in Fig. $\mathbf{6 b}, \mathbf{i}, \mathbf{j}, \mathbf{l}, \mathbf{m}$ represent individual mice. 

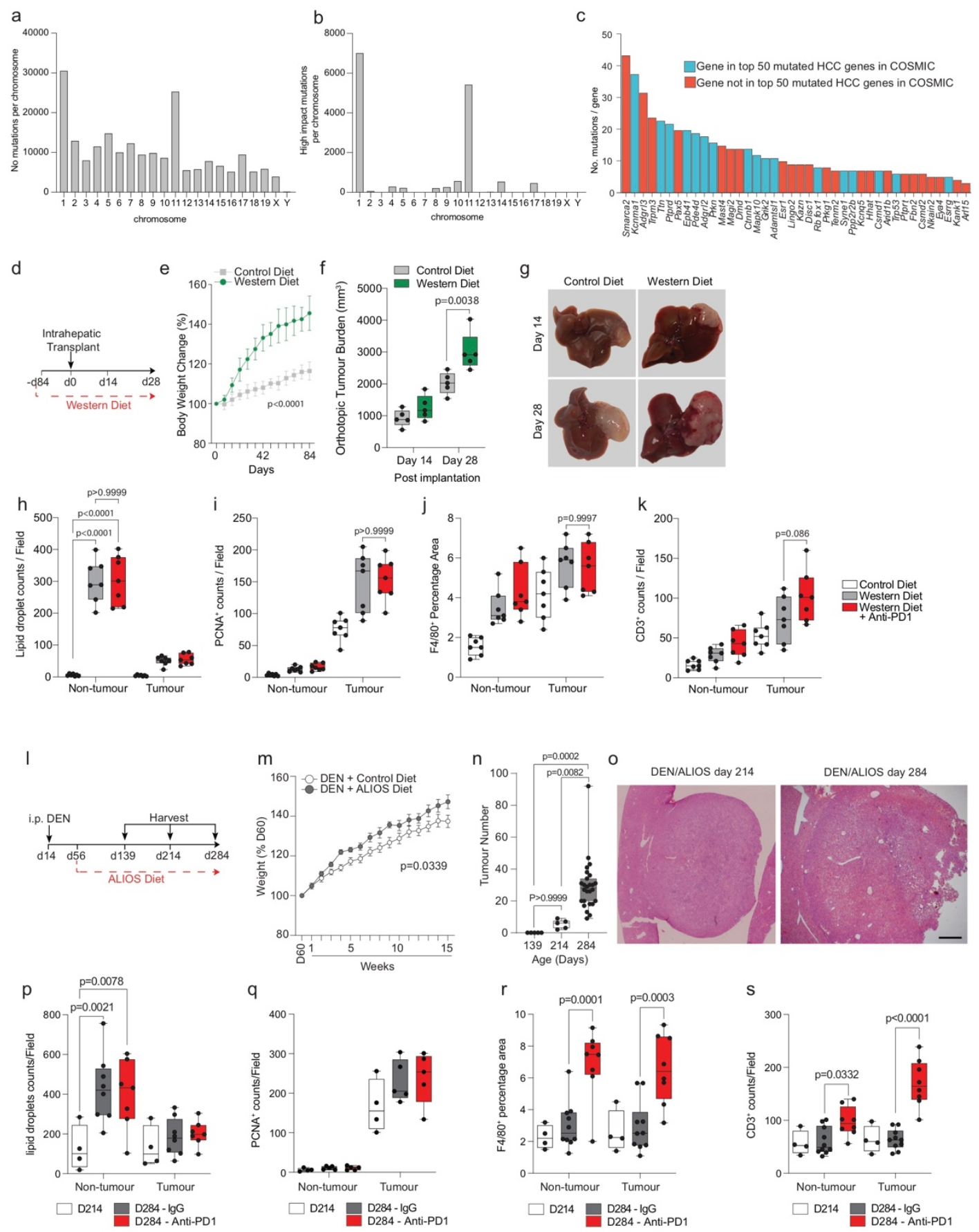

Supplementary Data Figure 1. Steatosis promotes tumour development and anti-PD1 resistance.

a) Number of mutations per chromosome in Hep53.4 HCC cells.

b) Number of high impact mutations per chromosome in Hep53.4 cells.

c) Top 40 mutated genes in Hep53.4 HCC cells, with high confidence human orthologues. Blue: gene in top 50 mutated HCC genes in the Catalogue of Somatic Mutations in Cancer (COSMIC) database. Red: gene not in top 50 mutated HCC genes in COSMIC.

d) Timeline schematic of the orthotopic NASH-HCC model.

e) Quantification of body weight change for mice fed a normal or Western diet, presented as percentage weight change compared with pre-diet weight. Control diet $n=14$ mice. Western diet $\mathrm{n}=14$ mice. Two-way ANOVA. Error bars represent Mean \pm SEM. 
f) Quantification of the tumour burden $\left(\mathrm{mm}^{3}\right)$ for the orthotopic NASH-HCC mice fed a control or Western diet at day 14 and day 28 post intra-hepatic injection. $n=5$ mice per condition. Two-way ANOVA with Sidak's multiple comparisons test.

g) Representative images of livers at day 14 and day 28 post-intrahepatic injection for the orthotopic NASH-HCC mice fed a control or Western diet. $n=5$ mice per condition.

h) Quantification of lipid droplet counts/field in non-tumour liver and tumour of the orthotopic NASH-HCC mice fed a control or Western diet and treated with IgG-control or anti-PD1, at day 28 post intrahepatic injection. $n=7$ mice per condition. Two-way ANOVA with Sidak's multiple comparisons test.

i) Quantification of PCNA counts/field in non-tumour liver and tumour for the orthotopic NASHHCC mice fed a control or Western diet and treated with IgG-control or anti-PD1, at day 28 post intrahepatic injection. $n=7$ mice per condition. Two-way ANOVA with Sidak's multiple comparisons test.

j) Quantification of $\mathrm{F} 4 / 80^{+}$as a percentage of area in non-tumour liver and tumour for the orthotopic NASH-HCC mice fed a control or Western diet and treated with IgG-control or antiPD1, at day 28 post intrahepatic injection. $n=7$ mice per condition. Two-way ANOVA with Sidak's multiple comparisons test.

k) Quantification of $\mathrm{CD}^{+}$counts/field in non-tumour liver and tumour for the orthotopic NASHHCC mice fed a control or Western diet and treated with IgG-control or anti-PD1, at day 28 post intrahepatic injection. $n=7$ mice per condition. Two-way ANOVA with Sidak's multiple comparisons test.

I) Timeline schematic of the autochthonous DEN/ALIOS NASH-HCC model.

m) Quantification of body weight change for mice injected with DEN at day 14 and fed a control or ALIOS diet from day 60, presented as a percentage of weight change compared with day 60. Control diet $n=11$ mice. ALIOS diet $n=9$ mice. Two-way ANOVA with Sidak's multiple comparisons test.

n) Quantification of tumour number for DEN/ALIOS mice at day 139, 214 and 284. Day $139 n=5$ mice; day $214 \mathrm{n}=5$ mice; day $284 \mathrm{n}=26$ mice. Kruskal-Wallis test with Dunn's multiple comparisons test.

o) Representative image of H\&E stained tumour nodules for DEN/ALIOS mice at day 214. $n=5$ mice. Scale bar $=1,000 \mu \mathrm{m}$.

p) Quantification of lipid droplet count/field for DEN/ALIOS mice at day 214 and for DEN/ALIOS mice treated with IgG-control or anti-PD1 at day 284. Day $214 n=4$ mice. Day $284 \lg G n=8$ mice. Day 284 anti-PD1 n=7 mice. Two-way ANOVA with Sidak's multiple comparisons test.

q) Quantification of PCNA ${ }^{+}$as counts/field for DEN/ALIOS mice at day 214 and for DEN/ALIOS mice treated with IgG-control or anti-PD1 at day 284. Day $214 n=4$ mice. $\lg G n=5$ mice. AntiPD1 $n=5$ mice. Two-way ANOVA with Sidak's multiple comparisons test.

r) Quantification of $\mathrm{F} 4 / 80^{+}$as percentage area for DEN/ALIOS mice at day 214 and for DEN/ALIOS mice treated with IgG-control or anti-PD1 at day 284. Day $214 n=4$ mice. Day $284 \lg n n=10$ mice. Day 284 anti-PD1 n=8 mice. Two-way ANOVA with Sidak's multiple comparisons test.

s) Quantification of $\mathrm{CD}^{+}$count/field for DEN/ALIOS mice at day 214 and for DEN/ALIOS mice treated with IgG-control or anti-PD1 at day 284. Day $214 n=4$ mice. Day $284 \lg \mathrm{n}=10$ mice. Day 284 anti-PD1 n=8 mice. Two-way ANOVA with Sidak's multiple comparisons test.

Dots in Supplementary Data Fig. 1f, i-k, m, n, p-s represent individual mice. 
a

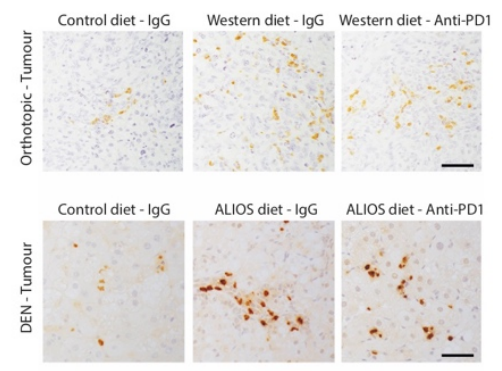

d

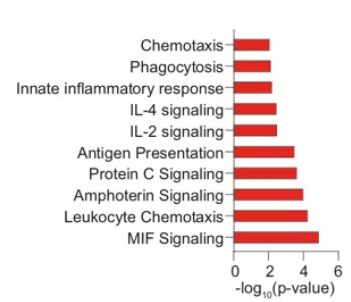

e
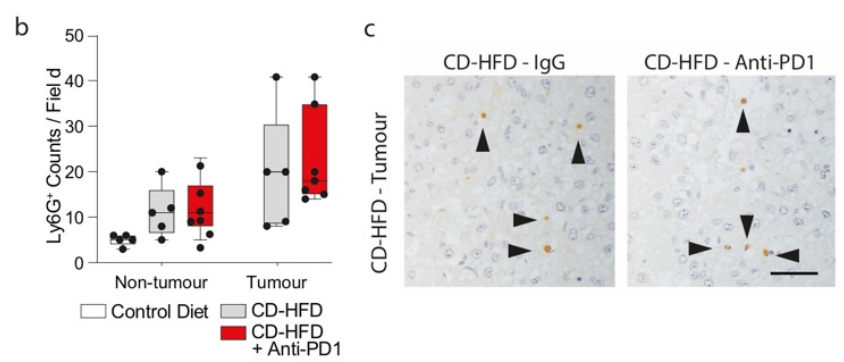

f
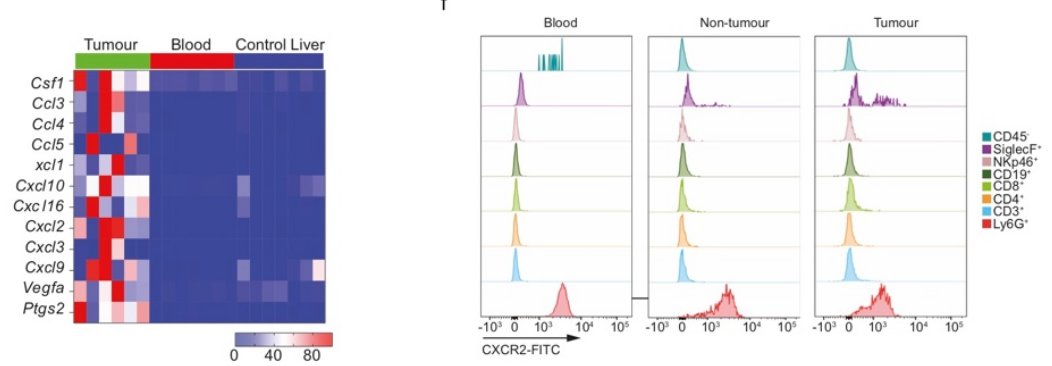

g

h

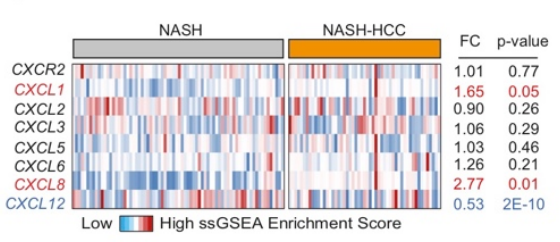

GO Terms: NASH F4 vs NASH F0/F1

positive regulation of neutrophil chemotaxis (GO:0090023)
positive regulation of neutrophil migration (GO:1902624) positive regulation of granulocyte chemotaxis (GO:0071624) -

chemokine-mediated signaling pathway (GO:0070098)-

regulation of neutrophil chemotaxis (GO:0090022)response to interleukin-1 (GO:0070555)-
positive regulation of chemotaxis (GO:0050921)regulation of chemotaxis (GO:0050921) neutrophil chemotaxis (GO:0030593)-
granulocyte chemotaxis (GO:0071621)granulocyte chemotaxis (GO:0071621) neutrophil migration (GO:1990266)
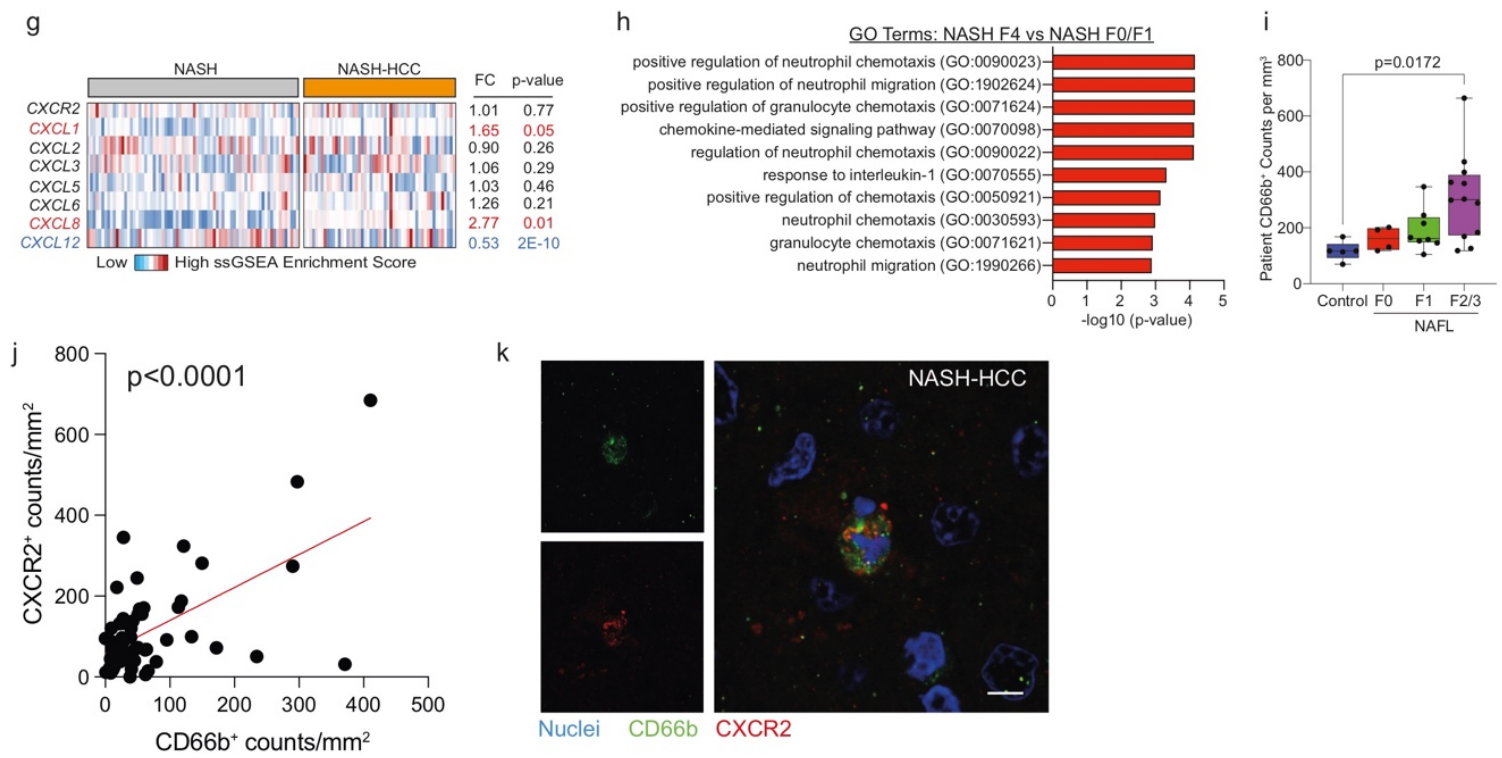

Supplementary Data Figure 2. Neutrophils associate with NASH-HCC in mouse models and humans

a) Representative image of $\mathrm{Ly}_{6 \mathrm{G}}{ }^{+}$neutrophil staining in tumours from the orthotopic NASH-HCC mice fed a control or western diet (top) and DEN mice fed a control or ALIOS diet (bottom). Orthotopic NASH-HCC: control diet + IgG-control $n=7$ mice; western diet + IgG-control $n=7$ mice; western diet + anti-PD1 $n=7$ mice. DEN/ALIOS: control diet + IgG-control $n=7$ mice; ALIOS diet + IgG-control $n=10$ mice; ALIOS diet + anti-PD1 $n=8$ mice. CD-HFD: IgG-control $n=5$ mice; CD-HFD + anti-PD1 $n=7$ mice. Scale bar $=100 \mu \mathrm{m}$.

b) Quantification of $\mathrm{Ly}_{6 \mathrm{G}}{ }^{+}$counts/field in non-tumour liver and tumours from IgG-control or antiPD1 treated choline-deficient high fat diet (CD-HFD) mice. Non-tumour: control diet $n=5$ mice; CD-HFD n=5 mice; CD-HFD + anti-PD1 n=7 mice. Tumour: CD-HFD n=5, CD-HFD + Anti-PD1 n=7. One-Way ANOVA with Tukey multiple comparisons test.

c) Representative images of $\mathrm{Ly}_{6 \mathrm{G}}{ }^{+}$neutrophil staining in tumours from CD-HFD mice treated with IgG-control or anti-PD1.

d) Top 10 process networks enriched in DEGs with increased expression in DEN/ALIOS TANs compared with peripheral blood and control liver $\mathrm{Ly}_{6 \mathrm{G}}{ }^{+}$neutrophils. 
e) Heatmap showing row-scaled expression of pro-tumour associated neutrophil genes in DEN/ALIOS TANs compared with peripheral blood and control liver neutrophils. Data are from bulk $\mathrm{Ly}_{6 \mathrm{G}}{ }^{+}$neutrophil RNA-Seq.

f) Representative histograms for CXCR2 staining in immune cell populations isolated from the peripheral blood, non-tumour liver and tumours of DEN/ALIOS mice. $n=3$ mice.

g) Heatmap showing row-scaled expression of $\mathrm{CxCl}$ chemokine transcripts, related to neutrophilfunction, for human NASH compared with NASH-HCC. Data are from bulk tumour RNA-Seq. NASH $n=74$ individuals; NASH-HCC $n=53$ individuals.

h) GO Terms enriched in advanced (F4) compared with early stage (FO/F1) NASH patients. Data are from patient resected tissue RNA-Seq. Data accessed from Govaere et al.

i) Quantification of $\mathrm{CD} 6 \mathrm{~b}^{+}$neutrophil count by IHC from NAFLD patient resected tissue. Control $n=5$ individuals; FO $n=4$ individuals; $F 1 n=8$ individuals; $F 2 / 3 n=12$ individuals.

j) Correlation of CD66b and CXCR2 counts from tumour and non-tumour regions of HCC patients.

k) Representative immunofluorescence of intra-tumour $\mathrm{CXCR2}{ }^{+} \mathrm{CD} 66 \mathrm{~b}^{+}$neutrophil from NASH$\mathrm{HCC}$ patient resected tissue. Nuclei $=$ blue; $C D 66 \mathrm{~b}=$ green; $C X C R 2=$ red. $n=5$ individuals. Scale bar $=10 \mu \mathrm{m}$.

Dots/columns in Supplementary Data Fig. 2e, g, i, j represent individual mice/patients. 

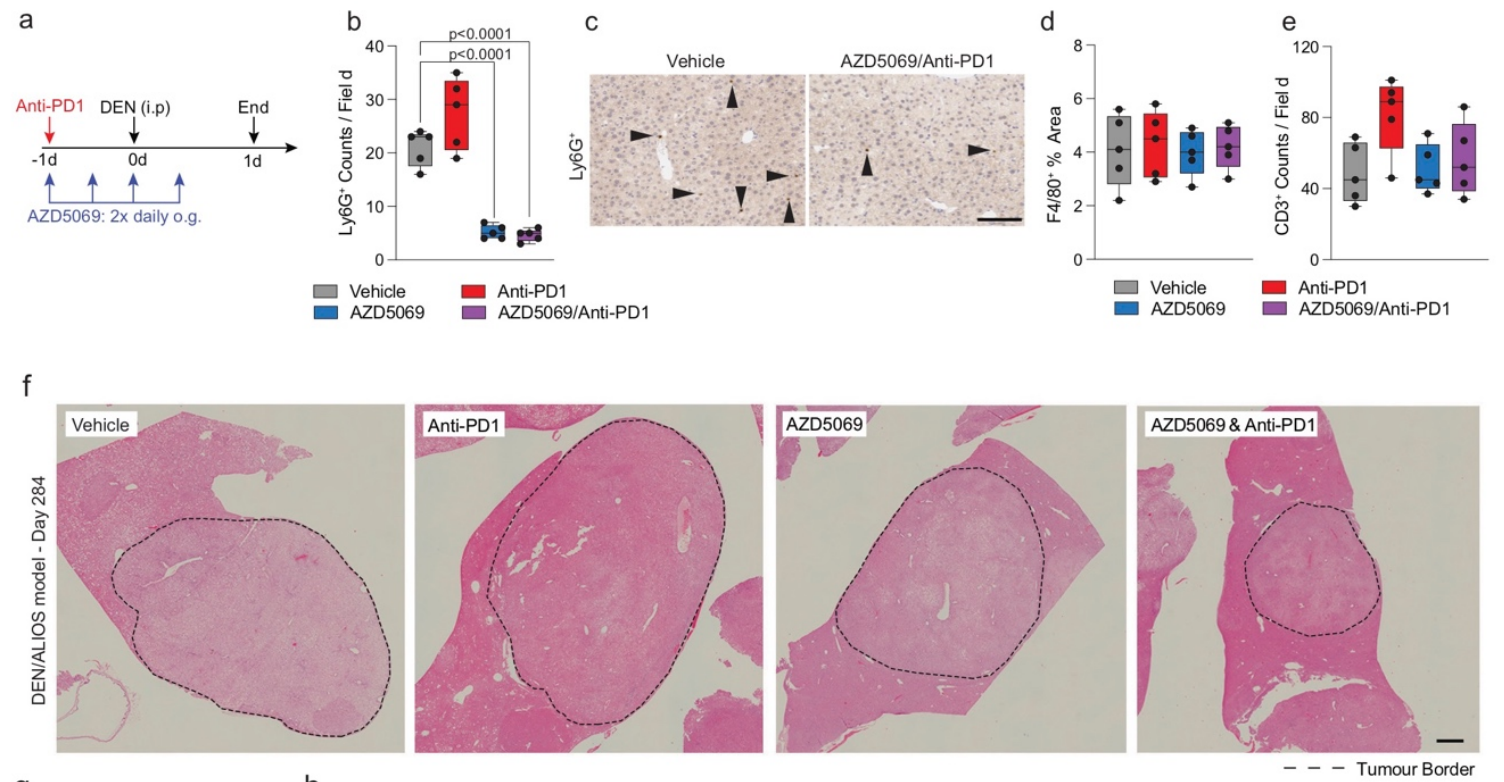

g

$\mathrm{h}$
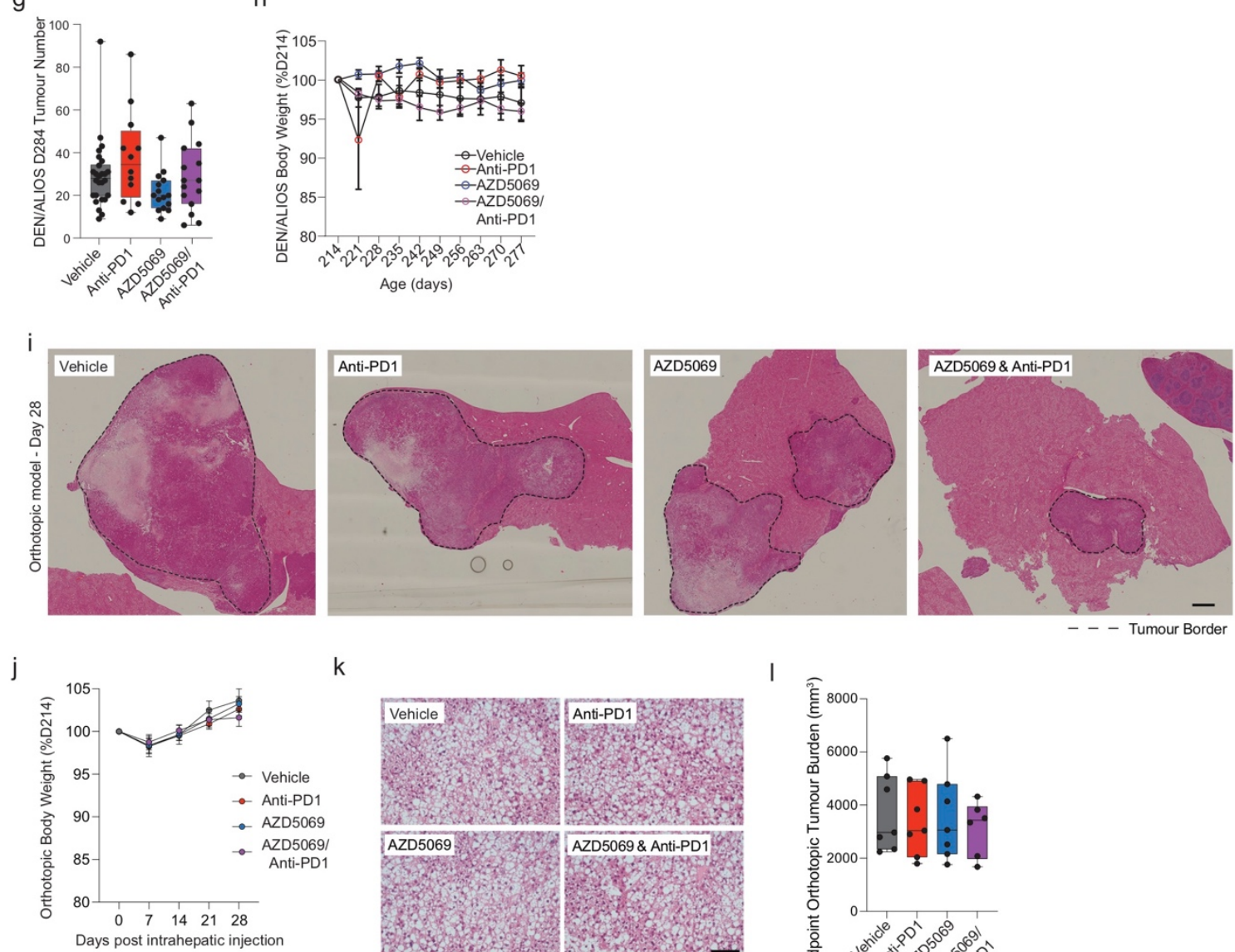

k
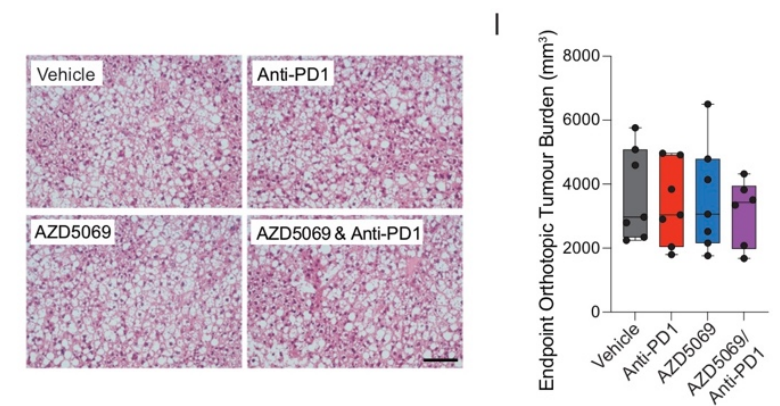

Supplementary Data Figure 3. CXCR2 inhibition alters neutrophil regulation and sensitises to antiPD1 therapy

a) Schematic for the acute-DEN model treatment regime.

b) Quantification of $\mathrm{Ly}_{6 \mathrm{G}}{ }^{+}$counts/field by $\mathrm{IHC}$ for the livers from acute-DEN mice treated with Vehicle-control, anti-PD1, AZD5069, or AZD5069/anti-PD1. $n=5$ mice per condition. One-Way ANOVA with Tukey's multiple comparisons test. 
c) Representative Ly $6 \mathrm{G}^{+}$staining of liver sections from acute-DEN mice. $\mathrm{n}=5$ mice per condition. Scale bar $=100 \mu \mathrm{m}$.

d) Quantification of $\mathrm{F} 4 / 80^{+}$macrophages as a percentage area of the field by $\mathrm{IHC}$ for the livers from acute-DEN mice treated with Vehicle-control, anti-PD1, AZD5069, or AZD5069/anti-PD1. $\mathrm{n}=5$ mice per condition. One-Way ANOVA with Tukey's multiple comparisons test.

e) Quantification of $\mathrm{CD}^{+}$counts/field by IHC for the livers from acute-DEN mice treated with Vehicle-control, anti-PD1, AZD5069, or AZD5069/anti-PD1. $n=5$ mice per condition. One-Way ANOVA with Tukey's multiple comparisons test.

f) Representative image of H\&E stained liver sections for DEN/ALIOS mice at day 284. Vehicle n=15 mice; anti-PD1 n=12 mice; AZD5069 n=15 mice; AZD5069/anti-PD1 n=15 mice. Scale bar $=1,000 \mu \mathrm{m}$.

g) Quantification of tumour number for DEN/ALIOS mice at day 284 for each treatment arm. Vehicle $\mathrm{n}=15$ mice; anti-PD1 n=12 mice; AZD5069 n=15 mice; AZD5069/anti-PD1 n=15 mice. Kruskal-Wallis test with Dunn's multiple comparisons test.

h) Quantification of body weight change for DEN/ALIOS mice, presented as percentage of weight change compared with pre-treatment start (day 214). Vehicle $n=16$ mice; anti-PD1 $n=14$ mice; AZD5069 n=14 mice; AZD5069/anti-PD1 n=13 mice. Error bars represent Mean \pm SEM.

i) Representative image of H\&E stained liver sections from the orthotopic NASH-HCC mice at day 28. $\mathrm{n}=12$ mice per condition. Scale bar $=1,000 \mu \mathrm{m}$.

j) Quantification of body weight change for the orthotopic NASH-HCC mice presented as a percentage of weight change compared with pre-intrahepatic injection. Error bars represent Mean \pm SEM. $n=12$ mice per condition.

k) Representative images of H\&E stained livers from orthotopic NASH-HCC mice. Vehicle $n=12$, Anti-PD1 n=12, AZD5069 n=12 and AZD5069/Anti-PD1 n=12 mice. Scale bar $=100 \mu \mathrm{m}$.

I) Quantification of tumour burden $\left(\mathrm{mm}^{3}\right)$ at end-point for the orthotopic NASH-HCC mice. Vehicle $n=7$ mice; anti-PD1 $n=7$ mice; AZD5069 $n=7$ mice; AZD5069/anti-PD1 $n=6$ mice.

Dots in Supplementary Data Fig. $\mathbf{3 b}, \mathbf{c}, \mathbf{d}, \mathbf{g}, \mathbf{l}, \mathbf{m}$ represent individual mice. 


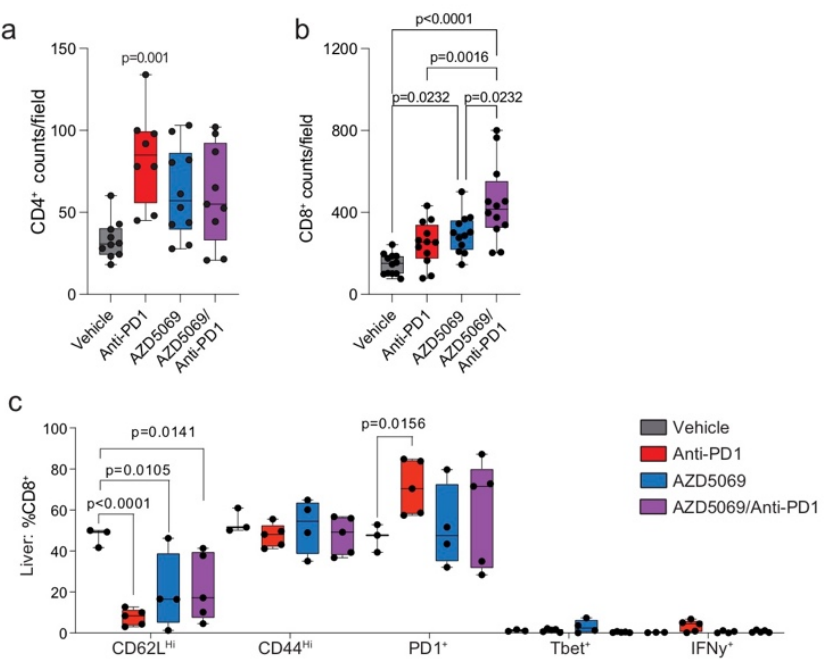

d

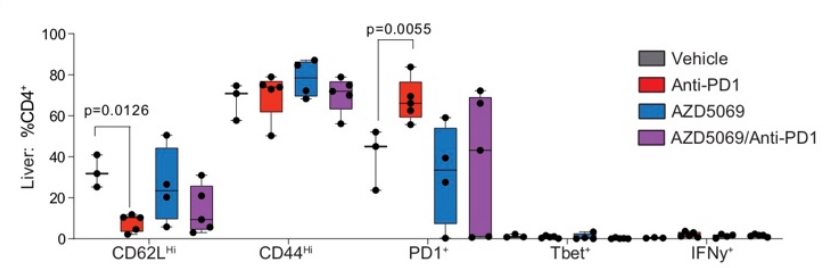

e
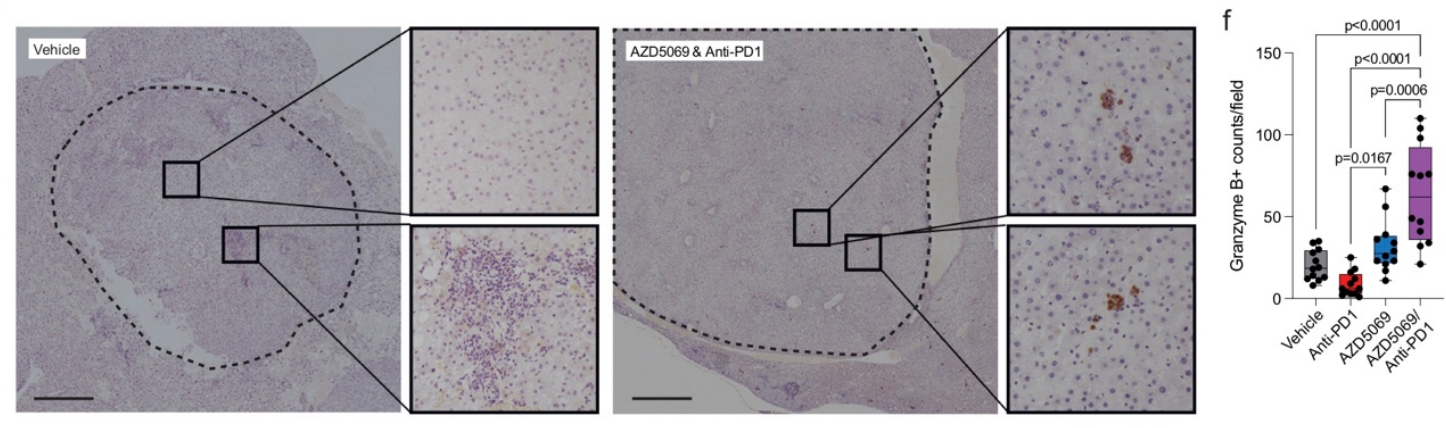

g
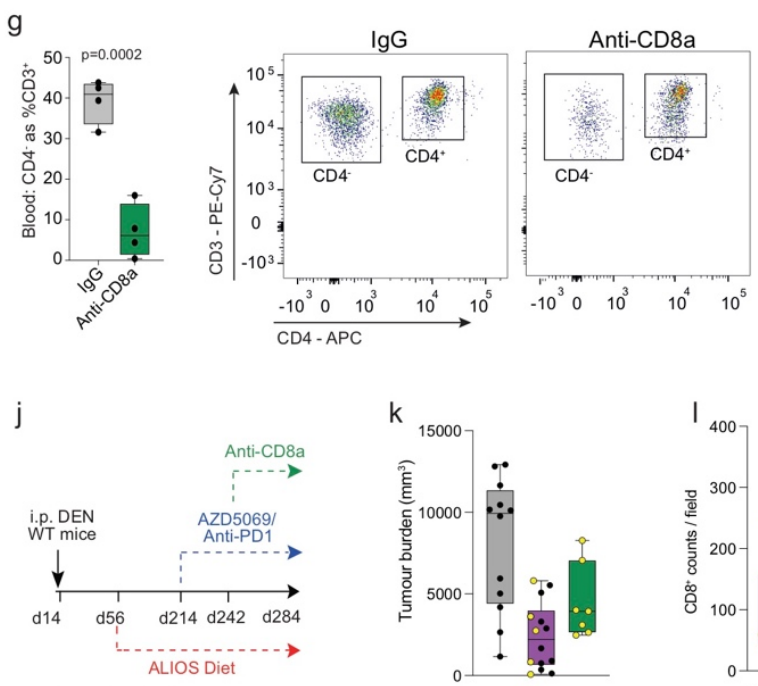

- Vehicle - AZD5069/Anti-PD1
AZD5069/Anti-PD1 + lgG isotype
AZD5069/Anti-PD 1 + Anti-CD8a $\mathrm{h}$
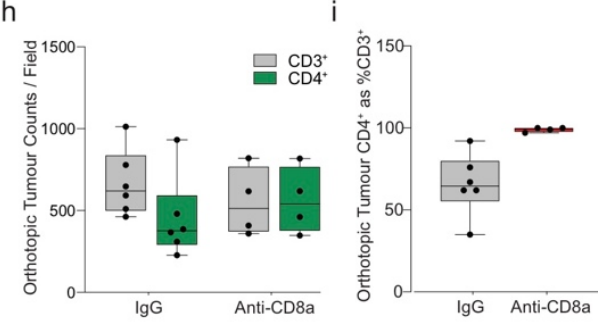

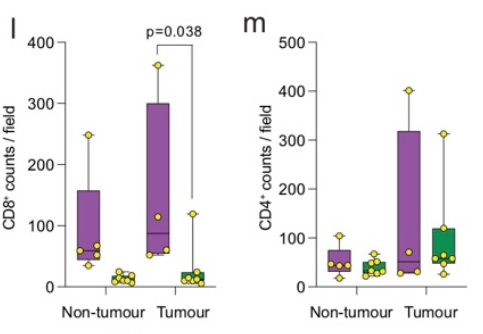

뭄 AZD5069/Anti-PD $1+\lg$ isotype 
Supplementary Data Figure 4. Cytotoxic CD8 ${ }^{+}$T cells contribute to combined AZD5069 \& anti-PD1 anti-tumour phenotype

a) Quantification of $\mathrm{CD} 8^{+}$counts/field in tumours DEN/ALIOS model for each treatment arm. Vehicle $n=10$ mice; anti-PD1 $n=8$ mice; AZD5069 n=10 mice; AZD5069/anti-PD1 n=9 mice. One-Way ANOVA with Tukey multiple comparisons test.

b) Quantification of $\mathrm{CD}^{+}$counts/field in livers for the orthotopic NASH-HCC mice for each treatment arm. Vehicle $n=12$ mice; anti-PD1 n=12 mice; AZD5069 n=12 mice; AZD5069/antiPD1 n=12 mice. One-Way ANOVA with Tukey's multiple comparisons test.

c) Quantification of $\mathrm{CD}^{+} \mathrm{CD} 8^{+}$cell surface phenotyping for DEN/ALIOS mice from each treatment arm at day 284. Vehicle $n=3$ mice; anti-PD1 $n=5$ mice; AZD5069 $n=4$ mice; AZD5069/anti-PD1 $\mathrm{n}=5$ mice. Two-way ANOVA with Dunnett's multiple comparisons test.

d) Quantification of $\mathrm{CD}^{+} \mathrm{CD} 4^{+}$cell surface phenotyping for $\mathrm{DEN} / \mathrm{ALIOS}$ mice from each treatment arm at day 284. Vehicle $n=3$ mice; anti-PD1 $n=5$ mice; AZD5069 $n=4$ mice; AZD5069/anti-PD1 $\mathrm{n}=5$ mice. Two-way ANOVA with Dunnett's multiple comparisons test.

e) Representative images of granzyme $B^{+}$clusters in Vehicle-control and AZD5069/anti-PD1 treated DEN/ALIOS livers. Scale bar $=1,000 \mu \mathrm{m}$.

f) Quantification of Granzyme $B^{+}$counts/field in livers for the orthotopic NASH-HCC mice for each treatment arm. Vehicle $n=12$ mice; anti-PD1 $n=12$ mice; AZD5069 $n=12$ mice; AZD5069/anti-PD1 n=12 mice. One-Way ANOVA with Tukey's multiple comparisons test.

g) Quantification of $\mathrm{CD}^{-}$as a percentage of $\mathrm{CD}^{+}$cells in the peripheral blood for the orthotopic NASH-HCC mice treated with AZD5069/anti-PD1 and IgG-control or anti-CD8 $\alpha$ (left) and representative flow cytometry plots (right). IgG $n=4$ mice; anti-CD8 $\alpha \mathrm{n}=4$ mice. Unpaired Ttest.

h) Quantification of $\mathrm{CD}^{+}$and $\mathrm{CD} 4^{+}$cells by $\mathrm{IHC}$ analysis in the tumours for the orthotopic NASH$\mathrm{HCC}$ mice treated with AZD5069/anti-PD1 and IgG-control or anti-CD8 $\alpha$. IgG-control $n=6$ mice; anti-CD $8 \alpha \mathrm{n}=4$ mice.

i) Quantification of $\mathrm{CD}_{4}^{+}$as a percentage of $\mathrm{CD}^{+}$cells in tumours for the orthotopic NASH-HCC mice treated with AZD5069/anti-PD1 and IgG-control or anti-CD8 $\alpha$. IgG-control $n=6$ mice; anti-CD $8 \alpha \mathrm{n}=4$ mice.

j) Timeline schematic for the anti-CD8a depletion regime in the authothonous DEN/ALIOS model.

k) Quantification of tumour burden $\left(\mathrm{mm}^{3}\right)$ for DEN/ALIOS mice at day 284 for each treatment arm. Vehicle $n=12$ mice (data from Figure 3b); AZD5069/anti-PD1 + no isotype IgG control $\mathrm{n}=12$ mice; AZD5069/anti-PD1 + isotype IgG control $\mathrm{n}=5$ mice; AZD5069/anti-PD1 + anti-CD8a $\mathrm{n}=7$ mice. One-way ANOVA with Tukey's multiple comparisons test.

I) Quantification of $C D 8^{+}$counts/field in non-tumour and tumour for DEN/ALIOS mice from each treatment arm. AZD5069/anti-PD1 + isotype IgG control $n=5$ mice; AZD5069/anti-PD1 + antiCD8a $n=7$ mice. Two-Way ANOVA with Tukey's multiple comparisons test.

m) Quantification of $C D 4^{+}$counts/field in non-tumour and tumour for DEN/ALIOS mice from each treatment arm. AZD5069/anti-PD1 + isotype IgG control $n=5$ mice; AZD5069/anti-PD1 + antiCD8a $n=7$ mice. Two-Way ANOVA with Tukey's multiple comparisons test. 
a
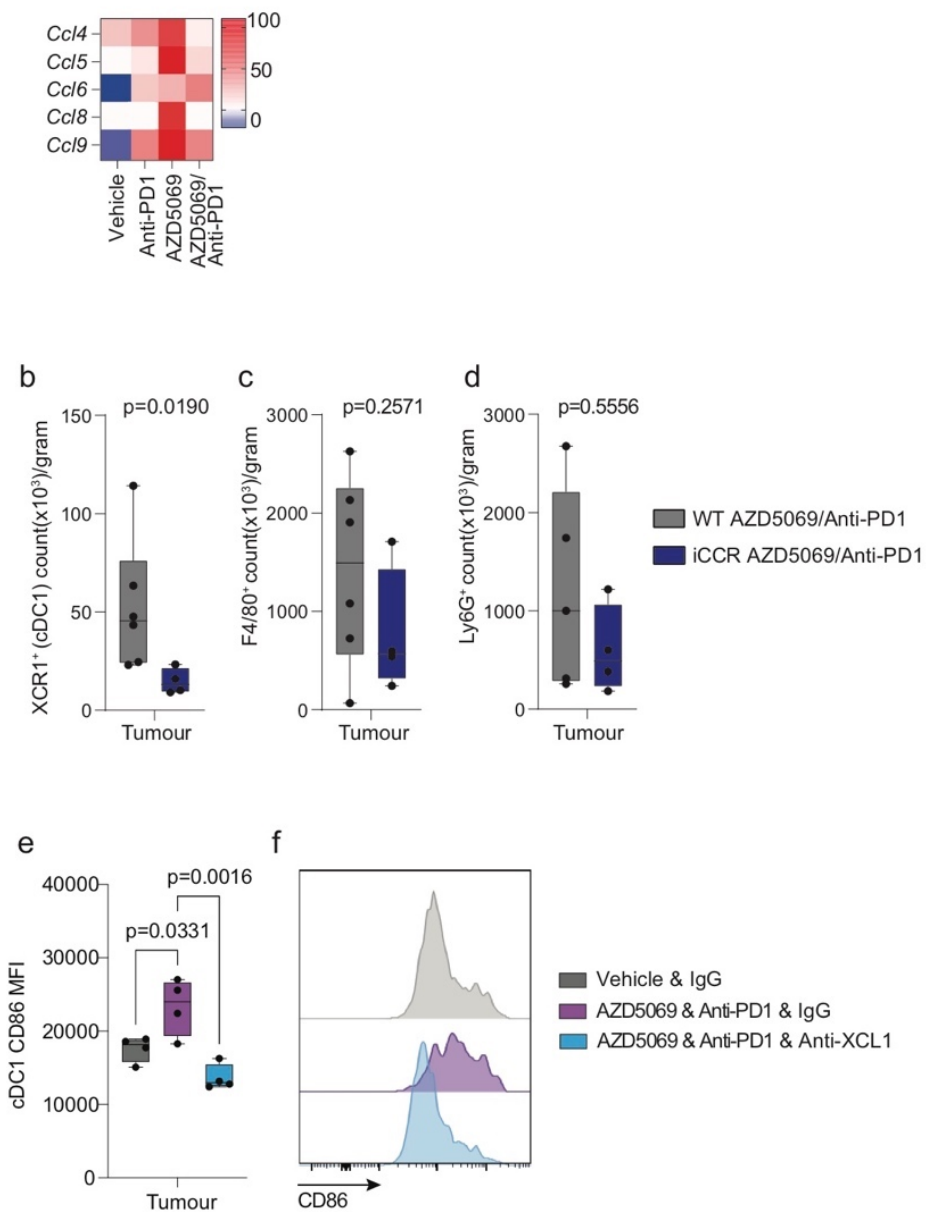

Vehicle \& IgG

- AZD5069 \& Ant-PD1 \& IgG

AZD5069 \& Anti-PD1 \& Anti-XCL1

Supplementary Data Figure 5. Dendritic cells contribute to combined AZD5069 \& anti-PD1 antitumour phenotype

a) Heatmap showing row-scaled expression of $\mathrm{Ccl}$ chemokine transcripts, related dendritic cell recruitment, in DEN/ALIOS treated groups. Data are from bulk tumour RNA-Seq.

b) Flow cytometric quantification of $X C R 1^{+} \mathrm{CDC1}$ counts $\left(x 10^{3}\right) / \mathrm{gram}$ in tumours of WT or iCCR genotype DEN/ALIOS mice treated with AZD5069/anti-PD1 at day 284. WT $n=6$. iCCR $n=4$. Mann-Whitney U-test.

c) Flow cytometric quantification of $\mathrm{F} 4 / 80^{+}$macrophage counts $\left(\times 10^{3}\right) /$ gram in tumours of WT or iCCR genotype DEN/ALIOS mice treated with AZD5069/anti-PD1 at day 284. WT $n=6$. iCCR $\mathrm{n}=4$. Mann-Whitney U-test.

d) Flow cytometric quantification of $\mathrm{Ly}_{6 \mathrm{G}}{ }^{+}$neutrophil counts $\left(\times 10^{3}\right) /$ gram in tumours of WT or iCCR genotype DEN/ALIOS mice treated with AZD5069/anti-PD1 at day 284. WT $n=6$. iCCR $n=4$. Mann-Whitney U-test.

e) Flow cytometric quantification CD86 median fluorescence intensity (MFI) of XCR1 ${ }^{+} \mathrm{CDC1}$ s from tumuour of orthtopic NASH-HCC mice. Vehicle $n=4$. AZD5069/anti-PD1 and IgG $n=4$. AZD5069/anti-PD1 and Anti-XCL1 $n=4$. One-Way ANOVA with Tukey multiple comparisons test.

f) Representative histogram plot of CD86 median fluorescence intensity (MFI) of XCR1 ${ }^{+} \mathrm{CDC} 1 \mathrm{~s}$ from tumuour of orthtopic NASH-HCC mice. Vehicle $n=4$. AZD5069/anti-PD1 and IgG $n=4$. AZD5069/anti-PD1 and Anti-XCL1 n=4.

Dots in Supplementary Data Fig. 6b-e, represent individual mice. 

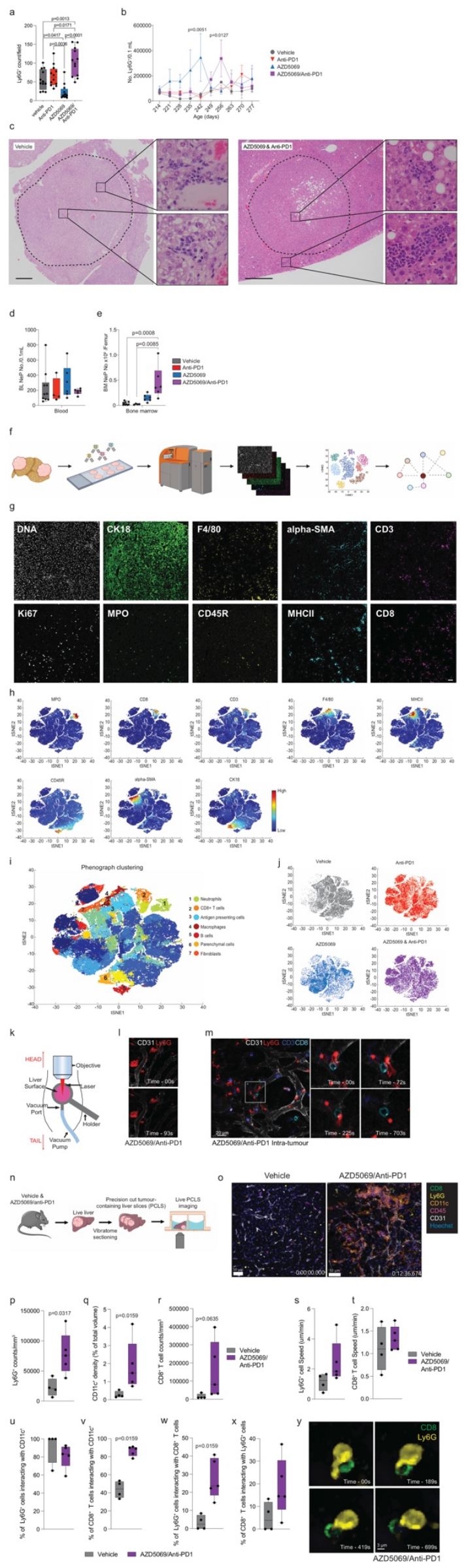
Supplementary Data Figure 6 - Imaging mass cytometry and live cell imaging reveals intra-tumour proliferating neutrophils associate and interact with $\mathrm{CD}^{+} \mathrm{T}$ cells and antigen presenting cells.

a) Quantification of $\mathrm{Ly}_{6 \mathrm{G}}{ }^{+}$counts/field in livers for the orthotopic NASH-HCC mice for each treatment arm. Vehicle $n=12$ mice; anti-PD1 n=12 mice; AZD5069 n=12 mice; AZD5069/antiPD1 n=12 mice. One-Way ANOVA with Tukey's multiple comparisons test.

b) Flow cytometric quantification of the number of $\mathrm{Ly} 6 \mathrm{G}^{+} / 0.1 \mathrm{~mL}$ peripheral blood from serial tail bleed analysis for DEN/ALIOS mice between day 214 and day 277. Vehicle: $n=6$ mice at all time-points except day 270 where $n=5$ mice. Anti-PD1: day 214-263 $n=5$ mice; day $270 n=3$ mice; day $277 n=4$ mice. AZD5069: $n=5$ mice at all time-points except day 242 where $n=4$ mice. AZD5069/anti-PD1: $n=5$ mice at all time-points except day 277 where $n=4$ mice. Two-way ANOVA with Tukey's multiple comparisons test. Error bars represent Mean $\pm \mathrm{SEM}$.

c) Representative images of H\&E staining in livers for DEN/ALIOS Vehicle and AZD5069/anti-PD1 treated mice. Clusters of neutrophils with a banded morphology are enlarged in AZD5069/anti-PD1 treated mice. Scale bar $=1,000 \mu \mathrm{m}$. $\mathrm{n}=9$ mice.

d) Flow cytometric quantification of NeP count in $0.1 \mathrm{ml}$ blood for DEN/ALIOS mice for each treatment arm at day 284. Vehicle $n=6$ mice; anti-PD1 $n=4$ mice; AZD5069 $n=6$ mice; AZD5069/anti-PD1 $n=6$ mice. Two-way ANOVA with Sidak's multiple comparisons test.

e) Flow cytometric quantification of NeP count per femur for DEN/ALIOS mice for each treatment arm at day 284. Vehicle $n=6$ mice; anti-PD1 $n=4$ mice; AZD5069 $n=6$ mice; AZD5069/anti-PD1 $\mathrm{n}=6$ mice. Two-way ANOVA with Sidak's multiple comparisons test.

1530

f) Schematic of Fluidigm Hyperion imaging mass cytometry pipeline, including preparation of tumour tissue microarray, metal conjugated antibody staining, imaging mass cytometry and downstream analysis including tSNE and neighbourhood analysis performed using histoCAT.

g) Representative images of hyperion staining for DNA intecalator, CK18, F4/80, alpha-SMA, CD3, Ki67, MPO, CD45R, MHCII and CD8. Scale bar $=100 \mu \mathrm{m} . \mathrm{n}=6$ tumours.

h) tSNE plots showing clustering of MPO, CD8, CD3, F4/80, MHCII, CD45R, alpha-SMA and CK18 positive cells.

i) TSNE plot showing phenograph clustering of cell populations across all images and treatment groups.

j) tSNE plots showing the contribution of individual treatment groups to clustering

k) Schematic for DEN/ALIOS model liver intravital microscopy (IVM) set-up.

l) Representative images for intra-tumour extravascular Ly6G+ clusters by IVM for DEN/ALIOS mice treated with AZD5069/anti-PD1 at day 284.

m) Representative images for intra-tumour IVM for DEN/ALIOS mice treated with AZD5069/antiPD1 at day 284 (right). Data are representative of $n=1$ mouse. Scale bar $=20 \mu \mathrm{m}$.

n) Schematic for DEN/ALIOS model precision cut tumour-containing liver slice (PCLS) microscopy set-up.

o) Representative images of live cell imaging of CD8, Ly6G, CD11c, CD45, CD31 and Hoechst in PCLS generated from DEN/ALIOS mice treated with either vehicle or AZD5069/anti-PD1 at day 284. Scale bar $=50 \mu \mathrm{m}$.

p) Quantification of $\mathrm{Ly}_{6 \mathrm{G}}{ }^{+}$counts $/ \mathrm{mm}^{3}$ in PCLS generated from DEN/ALIOS mice treated with either vehicle or AZD5069/anti-PD1 at day 284. Vehicle $n=4$ mice. AZD5069/anti-PD1 $n=5$ mice. Mann-Whitney U-test.

q) Quantification of $C D 11 c^{+}$density as a percentage of total volume in PCLS generated from DEN/ALIOS mice treated with either vehicle or AZD5069/anti-PD1 at day 284. Vehicle $n=4$ mice. AZD5069/anti-PD1 n=5 mice. Mann-Whitney U-test.

r) Quantification of $C D 8^{+} \mathrm{T}$ cell counts/mm3 in PCLS generated from DEN/ALIOS mice treated with either vehicle or AZD5069/anti-PD1 at day 284. Vehicle $n=4$ mice. AZD5069/anti-PD1 $n=5$ mice. Mann-Whitney U-test. 
s) Quantification of $\mathrm{Ly}_{6 \mathrm{G}} \mathrm{G}^{+}$speed in $\mu \mathrm{m} / \mathrm{min}$ in PCLS generated from DEN/ALIOS mice treated with either vehicle or AZD5069/anti-PD1 at day 284. Vehicle $n=4$ mice. AZD5069/anti-PD1 $n=5$ mice.

Quantification of $\mathrm{CD}^{+} \mathrm{T}$ cell speed in $\mu \mathrm{m} / \mathrm{min}$ in PCLS generated from DEN/ALIOS mice treated with either vehicle or AZD5069/anti-PD1 at day 284. Vehicle $n=4$ mice. AZD5069/antiPD1 $n=5$ mice.

u) Quantification of the percentage of $\mathrm{Ly} 6 \mathrm{G}^{+}$cells interacting with $\mathrm{CD} 11 \mathrm{c}^{+}$cell surface in PCLS generated from DEN/ALIOS mice treated with either vehicle or AZD5069/anti-PD1 at day 284. Vehicle $n=4$ mice. AZD5069/anti-PD1 $n=5$ mice.

v) Quantification of the percentage of $\mathrm{CD} 8^{+} \mathrm{T}$ cells interacting with $\mathrm{CD} 11 \mathrm{c}^{+}$cell surface in PCLS generated from DEN/ALIOS mice treated with either vehicle or AZD5069/anti-PD1 at day 284. Vehicle $n=4$ mice. AZD5069/anti-PD1 $n=5$ mice. Mann-Whitney U-test.

w) Quantification of the percentage of $\mathrm{Ly}_{6 \mathrm{G}}{ }^{+}$cells interacting with $\mathrm{CD} 8^{+} \mathrm{T}$ cells in PCLS generated from DEN/ALIOS mice treated with either vehicle or AZD5069/anti-PD1 at day 284. Vehicle $\mathrm{n}=4$ mice. AZD5069/anti-PD1 $\mathrm{n}=5$ mice. Mann-Whitney U-test.

$\mathrm{x}$ ) Quantification of the percentage of $\mathrm{CD}^{+} \mathrm{T}$ cells interacting with Ly6G $\mathrm{G}^{+}$cells in PCLS generated from DEN/ALIOS mice treated with either vehicle or AZD5069/anti-PD1 at day 284. Vehicle $\mathrm{n}=4$ mice. AZD5069/anti-PD1 $\mathrm{n}=5$ mice.

y) Representative images of a $\mathrm{Ly}_{6 \mathrm{G}^{+}}$neutrophil and $\mathrm{CD} 8^{+} \mathrm{T}$ cell interacting in PCLS generated from DEN/ALIOS mice treated with AZD5069/anti-PD1 at day 284. Scale bar $=3 \mu \mathrm{m}$. 
a

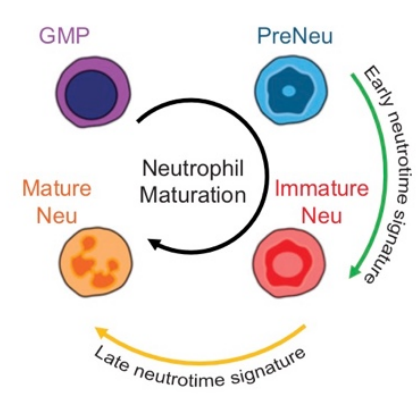

d

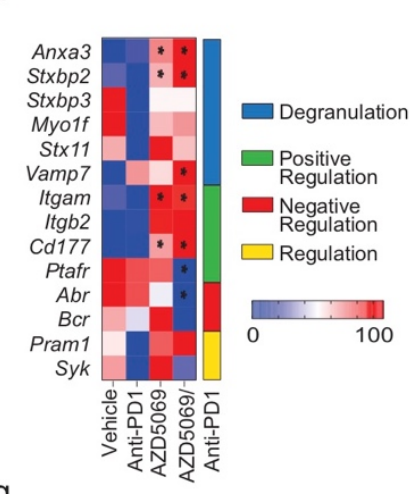

g

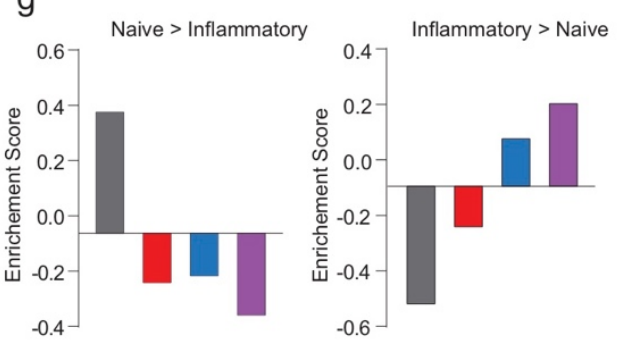

DEGs enriched in
mature Ly6G

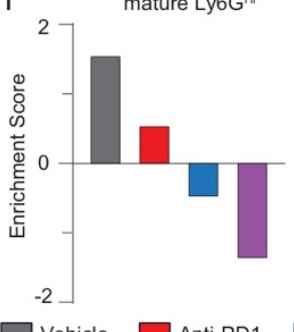

$\square$ Vehicle

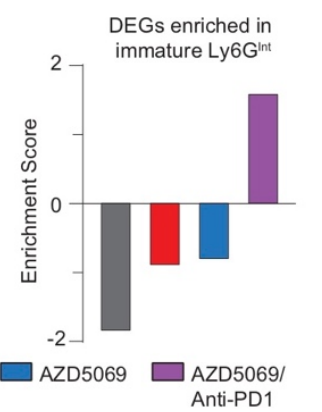

e
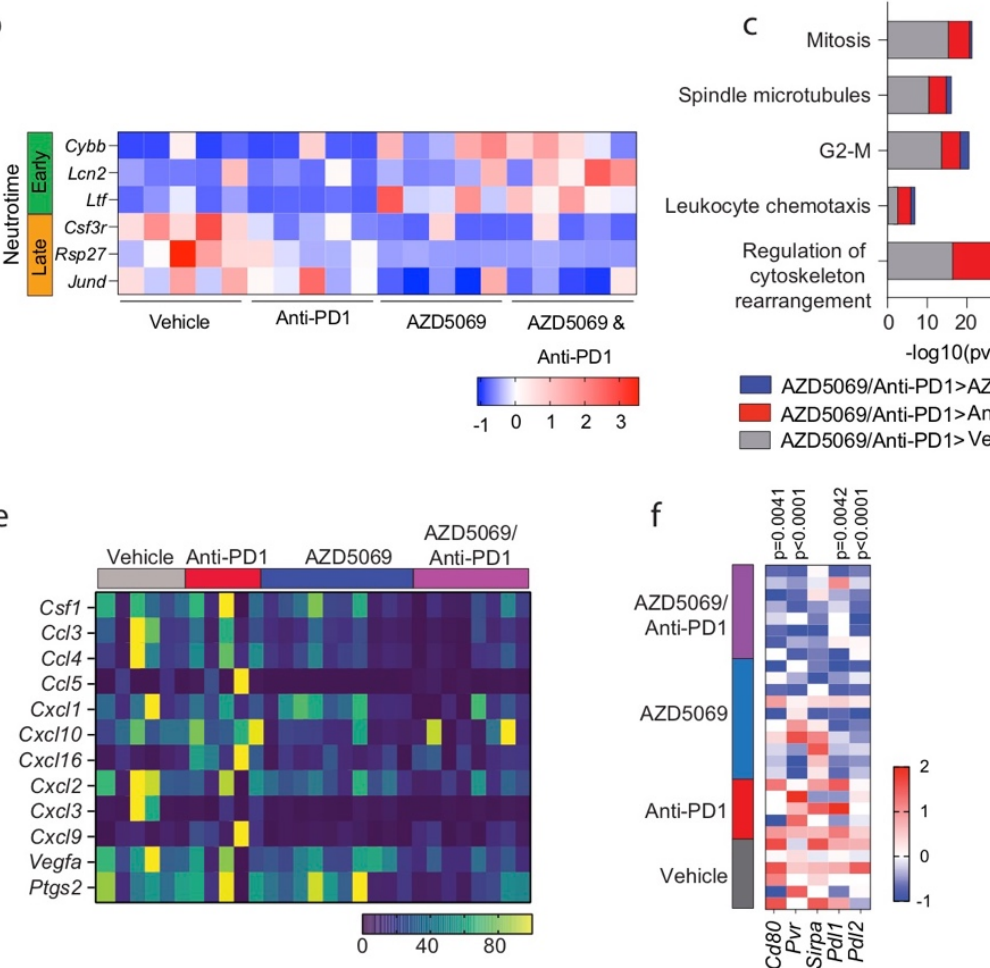

Leukocyte chemotaxis -

Regulation of
cytoskeleton

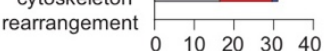
$-\log 10$ (pvalue)

AZD5069/Anti-PD1>AZD5069 - AZD5069/Anti-PD1>Anti-PD1 $\square$ AZD5069/Anti-PD1>Vehicle

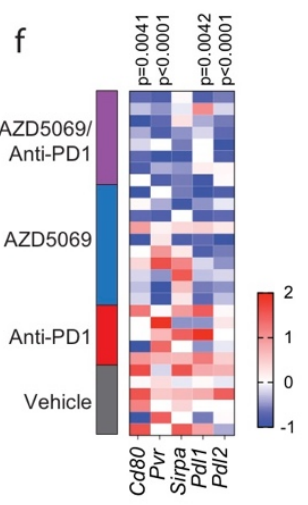

h

h Inflammatory GO Processes
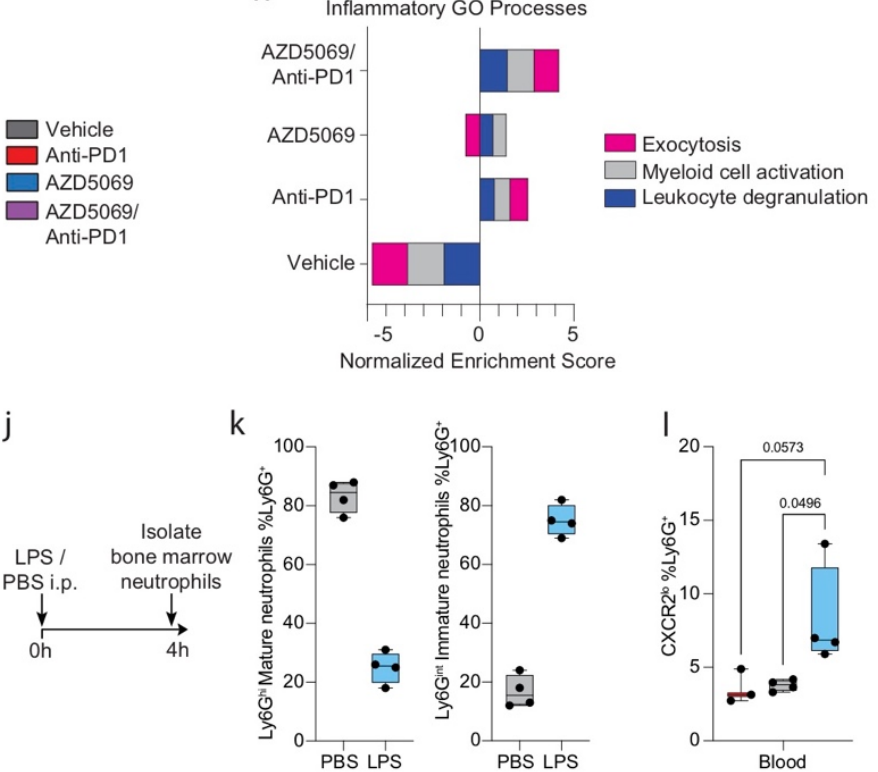

Anti-PD1

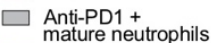

$\square$ Anti-PD1+

immature neutrophils

Supplementary Data Figure 7. Combined AZD5069 \& anti-PD1 therapy promotes an immature inflammatory tumour-associated neutrophil.

a) Schematic depicting neutrophil maturation stages classified according to early and late neutrotime signatures (as described by Grieshaber-Bouyer et al.).

b) Heatmap showing normalised expression of selected early and late neutrotime genes in neutrophils isolated from treated orthtoptic NASH-HCC mice at day 28. Data are from RT-PCR of isolated tumour neutrophils. $\mathrm{N}=5$ neutrophils isolations per group.

c) GSEA for process networks enriched in DEN/ALIOS TANs from AZD5069/anti-PD1 treated compared with Vehicle, anti-PD1 and AZD5069. Data are from bulk Ly6G ${ }^{+}$RNA-Seq. 
d) Heatmap showing row-scaled expression of genes associated with neutrophil degranulation GO Processes $(0043312,0043313,0043314,0043315)$ for DEN/ALIOS mice TANs. Data are from bulk Ly6G ${ }^{+}$TANs analysed by RNA-Seq.

e) Heatmap showing row-scaled expression of genes associated with a pro-tumour neutrophil phenotype for DEN/ALIOS TANs from each treatment arm. Data are from bulk Ly6G ${ }^{+}$TANs analysed by RNA-Seq.

f) Heatmap showing row-scaled expression of immune checkpoint genes for DEN/ALIOS TANs. Data are from bulk Ly6G ${ }^{+}$TANs analysed by RNA-Seq.

g) GSEA from DEGs increased in neutrophils isolated from PBS-control (left) and LPS-treated mice (right) for TANs isolated from DEN/ALIOS mice for each treatment arm. Data are from bulk Ly6G ${ }^{+}$TANs analysed by RNA-Seq.

h) GSEA for inflammatory GO Processes enriched in LPS-treated compared with PBS-control mice for TANs isolated from DEN/ALIOS mice for each treatment arm. Data are from bulk Ly6G ${ }^{+}$ TANs analysed by RNA-Seq.

i) GSEA for DEGs upregulated in LPS-treated peripheral blood, compared with PBS-controls, by mature Ly6G ${ }^{\mathrm{Hi}}$ neutrophils (left) and immature Ly6G $\mathrm{G}^{\text {int }}$ neutrophils (right) for DEN/ALIOS TANs. DEGs identified from bulk hepatic $\mathrm{Ly}_{6 \mathrm{G}}{ }^{+}$neutrophils from PBS-control and LPS-treated mice (Mackey et al., 2021) analysed by RNA-Seq, and GSEA from from bulk Ly6G+ TANs analysed by RNA-Seq.

j) Schematic showing timeline of treatment of WT mice with LPS or PBS for harvesting of immature and mature neutrophils.

k) Flow cytometric quantification of Ly6G $\mathrm{G}^{\text {hi }}$ and $\mathrm{Ly} 6 \mathrm{G}^{\text {int }}$ neutrophils as a percentage of total Ly6G ${ }^{+}$ bone marrow cells from LPS and PBS treated mice. LPS $n=4$. PBS $n=4$.

I) Flow cytometric quantification of $\mathrm{CXCR2} 2^{\mathrm{Lo}}$ neutrophils as a percentage of $\mathrm{Ly} 6 \mathrm{G}^{+}$neutrophils from the blood of orthotopic NASH-HCC, neutrophil/anti-PD1 therapy mice at day 28. Anti$P D 1 n=3$. Anti-PD1/Mature neutrophils $n=4$. Anti-PD1/Immature neutrophils $n=4$.

Bulk DEN/ALIOS Ly6G ${ }^{+}$TAN RNA-Seq data in Supplementary Data Fig. 7b-h: Vehicle $n=6$ mice; antiPD1 $n=5$ mice, AZD5069 n=10 mice; AZD5069 n=8 mice. Bulk hepatic Ly6G ${ }^{+}$neutrophil RNA-Seq data in Supplementary Data Fig. 7 from Mackey et al., 2021.

Dots in Supplementary Data Fig. 7j-q represent individual mice.

\section{Supplementary Movie - Neutrophils associate and interact with $\mathrm{CD8}{ }^{+} \mathrm{T}$ cells and $\mathrm{CD} 11 \mathrm{c}^{+}$cells.}

Representative time-lapse movies of tumour bearing precision cut liver slices generated from livers from DEN/ALIOS mice treated with either vehicle or AZD5069/anti-PD1 at day 284. Samples were stained for CD8 (green), Ly6G (yellow), CD11c (orange), CD45 (purple), CD31 (white) and Hoechst (blue) and imaged for $15-40 \mathrm{~min}$ in a full incubation chamber at $37^{\circ} \mathrm{C}$ with $5 \% \mathrm{CO}$. Scale bar $=50 \mu \mathrm{m}$.

Supplementary table 1: List of mouse primer sequences used for qRT-PCR analysis.

\begin{tabular}{|c|c|c|}
\hline & Forward primer & Reverse primer \\
\hline$c y b b$ & GATGATAGCACTGCACACCG & ATTCCTGTGATCCCAGCCAA \\
\hline$L c n 2$ & AAGGTGGCAGAACGAGATGA & ACCGCATAGTAGTGAGTCCG \\
\hline$L t f$ & ACTGAATGGGTGGTGAGTGT & GGGAGTGCTGGCCAAATAAG \\
\hline$C s f 3 r$ & AAGACCCCAGGAGACCTTTG & GCCAGAGACAGAGACACACT \\
\hline Rsp27 & GACGTGAAATGCCCAGGATG & CTTTCAGTGCTGCTTCCTCC \\
\hline Jund & CACGCTCTGCCTTTCCTTTT & AAAGAGAGGGGATGGTGTCG \\
\hline
\end{tabular}

